\title{
ON THE COMPOSITION OF YOUNG, DIRECTLY IMAGED GIANT PLANETS
}

\author{
J. I. Moses ${ }^{1}$, M. S. Marley ${ }^{2}$, K. Zahnle ${ }^{2}$, M. R. Line ${ }^{3}$, J. J. Fortney ${ }^{3}$, T. S. Barman ${ }^{4}$, \\ C. VISSCHER ${ }^{1,5}$, N. K. LEWIS ${ }^{6}$, AND M. J. WOLFF ${ }^{1}$ \\ ${ }^{1}$ Space Science Institute, 4750 Walnut Street, Suite 205, Boulder, CO 80301, USA; jmoses@ spacescience.org \\ ${ }^{2}$ NASA Ames Research Center, Moffett Field, CA 94035, USA \\ ${ }^{3}$ Department of Astronomy and Astrophysics, University of California, Santa Cruz, CA 95064, USA \\ ${ }^{4}$ Lunar and Planetary Laboratory, University of Arizona, Tucson, AZ 85721, USA \\ ${ }^{5}$ Dordt College, Sioux Center, IA 51250, USA \\ ${ }^{6}$ Space Telescope Science Institute, Baltimore, MD 21218, USA \\ Received 2016 April 14; revised 2016 July 28; accepted 2016 August 16; published 2016 September 23
}

\begin{abstract}
The past decade has seen significant progress on the direct detection and characterization of young, self-luminous giant planets at wide orbital separations from their host stars. Some of these planets show evidence for disequilibrium processes like transport-induced quenching in their atmospheres; photochemistry may also be important, despite the large orbital distances. These disequilibrium chemical processes can alter the expected composition, spectral behavior, thermal structure, and cooling history of the planets, and can potentially confuse determinations of bulk elemental ratios, which provide important insights into planet-formation mechanisms. Using a thermo/photochemical kinetics and transport model, we investigate the extent to which disequilibrium chemistry affects the composition and spectra of directly imaged giant exoplanets. Results for specific "young Jupiters" such as HR 8799 b and 51 Eri b are presented, as are general trends as a function of planetary effective temperature, surface gravity, incident ultraviolet flux, and strength of deep atmospheric convection. We find that quenching is very important on young Jupiters, leading to $\mathrm{CO} / \mathrm{CH}_{4}$ and $\mathrm{N}_{2} / \mathrm{NH}_{3}$ ratios much greater than, and $\mathrm{H}_{2} \mathrm{O}$ mixing ratios a factor of a few less than, chemical-equilibrium predictions. Photochemistry can also be important on such planets, with $\mathrm{CO}_{2}$ and $\mathrm{HCN}$ being key photochemical products. Carbon dioxide becomes a major constituent when stratospheric temperatures are low and recycling of water via the $\mathrm{H}_{2}+\mathrm{OH}$ reaction becomes kinetically stifled. Young Jupiters with effective temperatures $\lesssim 700 \mathrm{~K}$ are in a particularly interesting photochemical regime that differs from both transiting hot Jupiters and our own solar-system giant planets.
\end{abstract}

Key words: planetary systems - planets and satellites: atmospheres - planets and satellites: composition - planets and satellites: gaseous planets - planets and satellites: individual (51 Eri b, HR 8799 b)

Supporting material: tar.gz file

\section{INTRODUCTION}

Most of the exoplanets discovered to date have been identified through transit observations or radial-velocity measurements-techniques that favor the detection of large planets orbiting close to their host stars. Direct detection of a planet within the overwhelmingly glare and non-negligible point-spread function of its brighter star is challenging and requires high-contrast observations, often with adaptive-optics techniques from large telescopes on the ground or in space. As a result of these observational challenges, direct imaging favors the detection of massive, self-luminous (i.e., young) giant planets at wide orbital separations from their host stars. These "young Jupiters" are hot at depth because the leftover accretional and gravitational potential energy from the planet's formation has not had time to convect up through the atmosphere and be radiated away yet. Only $\sim 3 \%$ of the currently confirmed exoplanets ${ }^{7}$ have been detected through direct imaging, but these planetary systems have high intrinsic interest. For example, they serve as potential analogs to our own solar system in its formative years, when Jupiter and our other giant planets were born and evolved behind ice condensation fronts in the solar nebula but never migrated

\footnotetext{
7 See http://exoplanet.eu, http://exoplanetarchive.ipac.caltech.edu, or http://www.openexoplanetcatalogue.com.
}

inward-unlike, apparently, many of the known close-in, transiting, extrasolar giant planets. Directly imaged planets therefore provide a window into our own past and provide important clues to our solar system's origin and evolution (see, e.g., Madhusudhan et al. 2014). Wavelength-dependent photometry or spectra of directly imaged planets can also provide useful constraints on atmospheric properties such as composition, thermal structure, metallicity, bulk elemental ratios, and the presence or absence of clouds (see the reviews of Bailey 2014; Madhusudhan et al. 2014, 2016; Crossfield 2015).

Short-period, transiting "hot Jupiters" and directly imaged "young Jupiters" both have similar effective temperatures, often ranging from $\sim 500$ to $2500 \mathrm{~K}$. However, in terms of their thermal structure and spectral appearance, directly imaged planets have more in common with brown dwarfs than with hot Jupiters (e.g., Burrows et al. 2003; Fortney et al. 2008b; Bowler 2016). In particular, the stratospheres (radiative regions) of directly imaged planets and brown dwarfs are much cooler than those of highly irradiated hot Jupiters, and the cooler regions overlying hot continuum regions at depth can result in potentially deeper molecular absorption bands being present in emission spectra (Madhusudhan et al. 2014). It can therefore be easier to detect atmospheric molecules on young Jupiters and brown dwarfs, unless high clouds are present to obscure the absorption features. 
One drawback of direct imaging is that the planet's radius and mass cannot be well determined, unlike the situation with, respectively, transit observations and radial-velocity measurements. Instead, the mass and radius of directly imaged planets are more loosely constrained through atmospheric modeling and comparisons with the observed luminosity and spectral/ photometric behavior, often in combination with estimates of the age of the system and constraints from evolutionary models. The theoretical modeling and model-data comparisons can result in degeneracies between the planet's apparent size, surface gravity, effective temperature, and cloud properties (e.g., Marley et al. 2007, 2012; Barman et al. 2011a, 2011b, 2015; Currie et al. 2011; Madhusudhan et al. 2011; Spiegel \& Burrows 2012; Bonnefoy et al. 2013, 2016; Lee et al. 2013; Skemer et al. 2014; Baudino et al. 2015; Morzinski et al. 2015; Zurlo et al. 2016).

On the other hand, the identification of molecular features in the observed spectra is typically unambiguous on young Jupiters (e.g., Konopacky et al. 2013; Barman et al. 2015), and $\mathrm{H}_{2} \mathrm{O}$, CO, and/or $\mathrm{CH}_{4}$ have been detected in in spectra from several directly imaged planets (Patience et al. 2010; Barman et al. 2011a, 2011b, 2015; Janson et al. 2013; Konopacky et al. 2013; Oppenheimer et al. 2013; Snellen et al. 2014; Chilcote et al. 2015; Macintosh et al. 2015). The apparent deficiency of methane features on many cooler directly imaged planets, in conflict with chemical equilibrium expectations, has been argued as evidence for disequilibrium processes like transport-induced quenching on these planets (e.g., Bowler et al. 2010; Hinz et al. 2010; Janson et al. 2010, 2013; Barman et al. 2011a, 2011b, 2015; Galicher et al. 2011; Marley et al. 2012; Skemer et al. 2012, 2014; Currie et al. 2014; Ingraham et al. 2014; Zahnle \& Marley 2014), and so many of the above groups included quenching in their theoretical modeling (see Visscher \& Moses 2011; Zahnle \& Marley 2014 for more details about $\mathrm{CO} \leftrightarrow \mathrm{CH}_{4}$ quenching on directly imaged planets and brown dwarfs). Other disequilibrium chemical processes such as photochemistry have been assumed to be unimportant due to the large orbital distances of these planets (Crossfield 2015); however, the young stellar hosts of directly imaged planets tend to be bright in the ultraviolet, making photochemistry potentially important (e.g., Zahnle et al. 2016).

The goal of the present investigation is to quantify the extent to which disequilibrium chemical processes like photochemistry and quenching affect the composition and spectra of young, directly imaged planets. Our main theoretical tool is a thermochemical-photochemical kinetics and transport model (e.g., Moses et al. 2011, 2013a, 2013b; Visscher \& Moses 2011) that tracks the chemical production, loss, and transport of the most abundant gas-phase species in a hydrogen-dominated planetary atmosphere. We calculate the expected composition of specific directly imaged exoplanets such as 51 Eri b and HR 8799 b, for which observational spectra are available, as well as investigate how the composition of generic "young Jupiters" is affected by planetary parameters such as the effective temperature, surface gravity, incident ultraviolet flux, and the strength of atmospheric mixing. We also explore how disequilibrium chemistry affects the resulting spectra of directly imaged planets.

\section{THEORETICAL MODEL}

To calculate the vertical profiles of atmospheric species on directly imaged planets, we use the Caltech/JPL KINETICS code (Allen et al. 1981; Yung et al. 1984) to solve the coupled one-dimensional (1D) continuity equations for 92 neutral carbon-, oxygen-, nitrogen-, and hydrogen-bearing species that interact through $\sim 1650$ kinetic reactions. Hydrocarbons with up to six carbon atoms are considered, although the reaction list becomes increasingly incomplete the heavier the molecule. We do not consider ion chemistry from photoionization (Lavvas et al. 2014) or galactic-cosmic-ray ionization (Rimmer et al. 2014). Ion chemistry is not expected to affect the mixing ratios of the dominant gas species, but it will likely augment the production of heavy organic molecules, just as on Titan (e.g., Vuitton et al. 2007; Waite et al. 2007). Lacking any definitive evidence to the contrary for directly imaged giant planets, we assume the atmospheres have a solar elemental composition.

The reaction list includes both "forward" (typically exothermic) reactions and their reverses, where the reverse reaction rate coefficient is calculated from the forward rate coefficient and equilibrium constant assuming thermodynamic reversibility (e.g., Visscher \& Moses 2011; Heng et al. 2016). All reactions except those involving photolysis are reversed. The fully reversed reaction mechanism ensures that thermochemical equilibrium is maintained kinetically in the hotter deep atmosphere, while disequilibrium photochemistry and transport processes can take over and dominate in the cooler upper atmosphere (e.g., Line et al. 2011; Moses et al. 2011; Kopparapu et al. 2012; Miller-Ricci Kempton et al. 2012; Venot et al. 2012; Agúndez et al. 2014a; Hu \& Seager 2014; Miguel \& Kaltenegger 2014; Benneke 2015; Zahnle et al. 2016). The model automatically accounts for the transport-induced quenching of species, whereby mixing ratios are "frozen in" at a constant mixing ratio above some quench pressure as vertical transport processes start to dominate over the chemical reactions that are attempting to drive the atmosphere back toward thermochemical equilibrium (Prinn \& Barshay 1977; Lewis \& Fegley 1984; Fegley \& Lodders 1994).

The quenching process depends on the adopted reaction mechanism (cf. Visscher et al. 2010b; Line et al. 2011; Moses et al. 2011; Visscher \& Moses 2011; Venot et al. 2012; Moses 2014; Zahnle \& Marley 2014; Wang et al. 2015; Rimmer \& Helling 2016). Our chemical reaction list is taken from Moses et al. (2013a) and includes a thorough review of the key reaction mechanisms of potential importance in the quenching of $\mathrm{CO} \leftrightarrows \mathrm{CH}_{4}$ and $\mathrm{N}_{2} \leftrightarrows \mathrm{NH}_{3}$ (Moses et al. 2010, 2011, 2013a, 2013b; Visscher et al. 2010b; Visscher \& Moses 2011; Moses 2014); further details of the thermo/ photochemical kinetics and transport model are provided in the above papers, and the reaction list is available as supporting material. Note that we do not include the fast rate coefficient for $\mathrm{H}+\mathrm{CH}_{3} \mathrm{OH} \rightarrow \mathrm{CH}_{3}+\mathrm{H}_{2} \mathrm{O}$ suggested by Hidaka et al. (1989) that is controlling $\mathrm{CO}-\mathrm{CH}_{4}$ quenching in the Venot et al. (2012) mechanism. As discussed by Norton \& Dryer (1990), Lendvay et al. (1997), and Moses et al. (2011), this reaction actually possesses a very high energy barrier $(>10,000 \mathrm{~K})$ and is not expected to be important under either methanolcombustion conditions or in the deep atmospheres of hydrogen-rich exoplanets-in other words, the Hidaka et al. rate coefficient greatly overestimates the rate of this reaction. 
Zahnle \& Marley (2014) adopt the upper limit for this reaction as suggested by Norton \& Dryer (1989) and find it to be important but not typically dominant in $\mathrm{CO}-\mathrm{CH}_{4}$ quenching, except for cooler brown dwarfs with weak mixing. We adopt the much smaller rate coefficient as calculated by Moses et al. (2011), and this reaction does not play a role in $\mathrm{CO}-\mathrm{CH}_{4}$ quenching. Similarly, we do not adopt the relatively fast ratecoefficient expression for $\mathrm{NH}_{2}+\mathrm{NH}_{3} \rightarrow \mathrm{N}_{2} \mathrm{H}_{3}+\mathrm{H}_{2}$ estimated by Konnov \& De Ruyck (2000) that is affecting $\mathrm{N}_{2}-\mathrm{NH}_{3}$ quenching in the Venot et al. (2012) mechanism, as again, this reaction is expected to have a high-energy barrier and be slower under relevant conditions than the Konnov and De Ruyck estimate (e.g., Dean et al. 1984).

Our model grids consist of 198 vertical levels separated uniformly in $\log$ (pressure) (providing multiple grid levels per scale height to insure accurate diffusion calculations), with a bottom level defined where the deep atmospheric temperature on an adiabatic gradient is greater than $\sim 2700 \mathrm{~K}$ (to insure that the $\mathrm{N}_{2}-\mathrm{NH}_{3}$ quench point is captured), and a top level residing at $\sim 10^{-8}$ mbar (to insure all the molecular absorbers are optically thin in the ultraviolet). The top region of our model grid extends through what would typically be the "thermosphere" of the planet; however, we neglect non-stellar sources of thermospheric heating (such as auroral and Joule heating), which are poorly understood but are important on our solarsystem giant planets (e.g., Yelle \& Miller 2004; Nagy et al. 2009). Our results should therefore only be considered reliable from the deep troposphere on up to the homopause level at the base of the thermosphere (near $10^{-4}$ to $10^{-6}$ mbar, depending on the strength of atmospheric mixing), where molecular diffusion acts to limit the abundance of heavy molecular and atomic species in the lighter background hydrogen atmosphere.

The thermal structure itself is not calculated self-consistently but is adopted from two different atmospheric models: (1) the radiative-convective equilibrium models described in McKay et al. (1989), Marley et al. (1999, 2002), and Saumon \& Marley (2008), with updates as described in Marley et al. (2012), and (2) the PHOENIX-based models described in Hauschildt et al. (1997), Allard et al. (2001), and Barman et al. (2011a), with updates as described in Barman et al. (2015). We add a smoothly varying, nearly isothermal profile at the top of the above-mentioned theoretical model profiles to extend our grids to lower pressures, except in isolated cases where we test the effects of a hotter $(1000 \mathrm{~K})$ thermosphere. Figure 1 shows the temperature profiles adopted for our cloud-free generic directly imaged planets, as a function of effective temperature $T_{\text {eff }}$ for two different assumed 1-bar surface gravities, $\log (g)=3.5$ and $4.0 \mathrm{cgs}$. These profiles are calculated without considering stellar irradiation-for all directly imaged planets discovered to date, the external radiation field has little effect on the thermal profile due to the planets' large orbital distance and strong internal heat flux. As such, the internal heat completely dominates the thermal structure, and temperatures on these planets are hotter at depth and colder in the stratospheric radiative region than for close-in transiting giant planets of the same effective temperature. The profiles from Figure 1 were generated with the NASA Ames brown-dwarf and exoplanet structure models (e.g., Marley et al. 2012); tables with the individual pressure-temperature structure from these models are available as supporting material. Disequilibrium processes like photochemistry and quenching are expected to have a

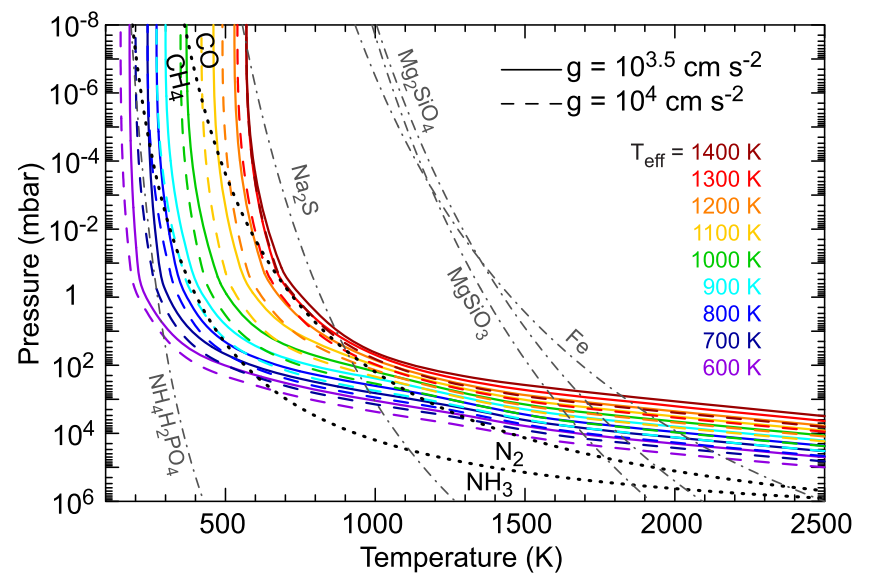

Figure 1. Theoretical temperature profiles for generic directly imaged planets from the radiative-convective equilibrium model of Marley et al. (2012), as a function of effective temperature $T_{\text {eff }}$ for an assumed surface gravity (in $\mathrm{cm} \mathrm{s}^{-2}$ ) of $\log (g)=3.5$ (colored solid lines) and $\log (g)=4.0$ (colored dashed lines) and assumed solar composition atmosphere in chemical equilibrium. Profiles are shown every $100 \mathrm{~K}$ from $T_{\text {eff }}=600$ to $1400 \mathrm{~K}$. The gray dot-dashed lines show the condensation curves for some important atmospheric cloud-forming species (as labeled) for an assumed solar-composition atmosphere. The thicker dotted black lines represent the boundaries where $\mathrm{CH}_{4}$ and $\mathrm{CO}$ have equal abundances and where $\mathrm{N}_{2}$ and $\mathrm{NH}_{3}$ have equal abundance in chemical equilibrium for solar-composition models. Methane and ammonia dominate to the lower left of these curves, while $\mathrm{CO}$ and $\mathrm{N}_{2}$ dominate to the upper right. Note that all the profiles remain within the CO-dominated regime at depth, whereas all except for the hottest planets transition to the $\mathrm{CH}_{4}$-dominated regime at higher altitudes.

relatively minor effect on the thermal structure (e.g., Agúndez et al. 2014b), unless these processes affect the $\mathrm{H}_{2} \mathrm{O}$ abundance.

Given a temperature-pressure profile, the NASA CEA code of Gordon \& McBride (1994) is then used to determine the chemical-equilibrium abundances, which are used as initial conditions in the photochemical model. We use the protosolar abundances listed in Table 10 of Lodders (2010) to define our "solar" composition. The mean molecular mass profile from the chemical-equilibrium solution, the pressure-temperature profile, and the assumed physical parameters of the planet become inputs to the hydrostatic equilibrium equation, whose solution sets the altitude scale and other atmospheric parameters along the vertical model grid. For a surface (1-bar) gravity of $g=10^{4}$ $\mathrm{cm} \mathrm{s}^{-2}$, the planet mass $M_{p}$ is $4 M_{J}$, and for $g=10^{3.5} \mathrm{~cm} \mathrm{~s}^{-2}$, $M_{p}=2 M_{J}$. For boundary conditions, we assume the fluxes of the species are zero at the top and bottom of the model. The models are run until steady state, with a convergence criterion of 1 part in 1000 . For the photochemical calculations, the atmospheric extinction is calculated from the absorption and multiple Rayleigh scattering of gases only-aerosol extinction is ignored due to a lack of current predictive capability regarding the hazes. The atmospheric radiation field for the photochemical model is calculated for diurnally averaged conditions for an assumed (arbitrary) $24 \mathrm{hr}$ rotation period at $30^{\circ}$ latitude at vernal equinox, with an assumed zero axial tilt for the planet. These assumptions should provide acceptable "global average" conditions for most young Jupiters.

As is standard in 1D photochemical models, we assume that vertical transport occurs through molecular and "eddy" diffusion, with the eddy diffusion coefficient profile $K_{z z}(z)$ being a free parameter. The molecular diffusion coefficients assumed in the model are described in Moses et al. (2000). Although vertical transport of constituents in real atmospheres 


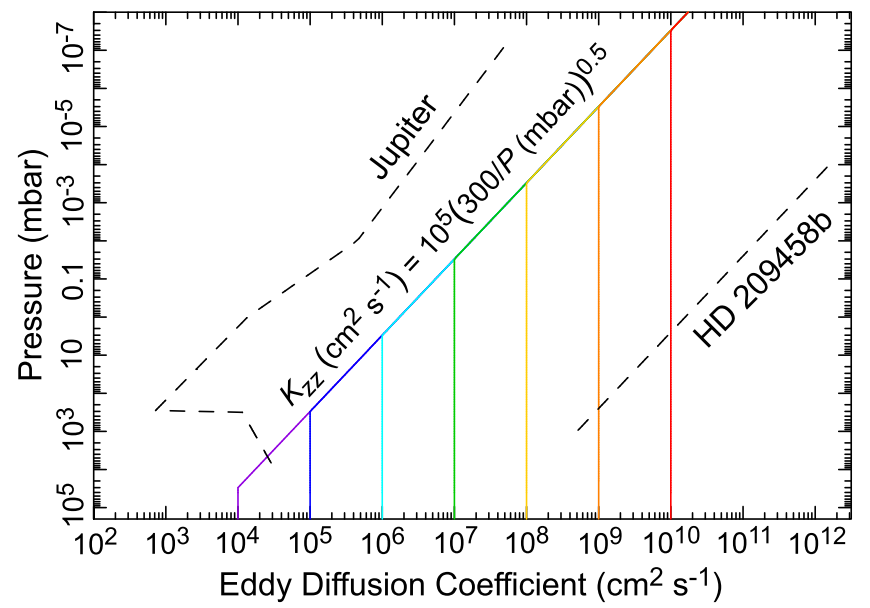

Figure 2. Eddy diffusion coefficient profiles (colored solid lines) adopted in our thermo/photochemical kinetics and transport models. The $K_{z z}$ profiles are assumed to vary as $10^{5}\left(300 / P_{\text {mbar }}\right)^{0.5} \mathrm{~cm}^{2} \mathrm{~s}^{-1}$ in the radiative region, with different models having different cutoff values $\left(K_{\text {deep }}\right)$ at depth. Profiles derived for Jupiter (Moses et al. 2005) and the hot Jupiter HD 209458b (Parmentier et al. 2013) are shown for comparison (dashed lines).

occurs through convection, large-scale advection, atmospheric waves, and turbulent "eddies" of all scales, this constituent transport often mimics diffusion (Lindzen 1981; Strobel 1981; Brasseur et al. 1999), and the concept of eddy diffusion has proven to be a useful one for atmospheric models. The eddy diffusion coefficient profile for an atmosphere cannot typically be derived accurately from first principles. Instead, observations of chemically long-lived species are used to empirically constrain $K_{z z}$ (z) (e.g., Allen et al. 1981; Atreya et al. 1984; Moses et al. 2005). On $\mathrm{H}_{2}$-dominated planets and brown dwarfs, the relative abundance of $\mathrm{CO}$ and $\mathrm{CH}_{4}$ can be used to constrain $K_{z z}$ at the quench point (see Prinn \& Barshay 1977; Fegley \& Lodders 1994; Griffith \& Yelle 1999; Visscher \& Moses 2011). For most directly imaged planets planets, the $\mathrm{CO}-\mathrm{CH}_{4}$ quench point will reside in the deep, convective portion of the atmosphere, where free-convection and mixinglength theories (e.g., Stone 1976) predict relatively large eddy diffusion coefficients and rapid mixing (e.g., $K_{z z} \gtrsim 10^{10} \mathrm{~cm}^{2}$ $\mathrm{s}^{-1}$ for most young Jupiters, assuming the atmospheric scale height as the mixing length). However, the mixing length to use for these expressions is not obvious (Smith 1998; Freytag et al. 2010), and the quench point for some planets may approach the radiative region, where $K_{z z}$ is expected to drop off significantly before increasing roughly with the inverse square root of atmospheric pressure due to the action of atmospheric waves (e.g., Lindzen 1981; Strobel 1981; Parmentier et al. 2013).

We therefore explore a range of possible $K_{z z}$ profiles, with roughly constant values at depth, trending to values that vary as $1 / \sqrt{P}$ as the pressure $P$ decreases. In particular, we assume that $K_{z z}\left(\mathrm{~cm}^{2} \mathrm{~s}^{-1}\right)=10^{5}\left(300 / P_{\mathrm{mbar}}\right)^{0.5}$ in the radiative region above $\sim 300$ mbar (hereafter called the stratosphere), but we do not let $K_{z z}$ drop below some value " $K_{\text {deep }}$ " that varies with the different models considered (see Figure 2). This convention allows the different models for a given $T_{\text {eff }}$ to have a similar homopause pressure level in the upper atmosphere (i.e., the pressure level to which the molecular species can be mixed before molecular diffusion starts to limit their abundance), while still testing the effect of variations in $K_{z z}$ at the quench point.
Note from Figure 2 that we have chosen stratospheric $K_{z z}$ profiles that are intermediate between those derived empirically from chemical tracers for our own solar-system (cold) Jupiter (Moses et al. 2005) and those derived from tracer transport in 3D dynamical models of the hot transiting exoplanet HD 209458b (Parmentier et al. 2013), which seems reasonable given that atmospheric temperatures for directly imaged planets are intermediate between the two. Eddy diffusion coefficients scale directly with vertical velocities and atmospheric length scales, and both tend to be larger for higher temperatures. Young Jupiters are very hot and convective at depth, but their stratospheres are relatively cold and statically stable.

When estimating $K_{z z}$ profiles for exoplanetary atmospheres, we keep in mind that atmospheric waves are typically responsible for mixing in the stratosphere (e.g., Lindzen 1981), and wave activity could be correlated with both the strength of stellar insolation and internal heat, as the main drivers for these waves. In the troposphere, convection dominates, and mixing is stronger for higher internal heat fluxes. For example, in the Freytag et al. (2010) hydrodynamic models of cool dwarfs, the maximum effective tropospheric diffusion coefficient (analogous to our " $K_{\text {deep }}$ ") increases with increasing $T_{\text {eff }}$ over the whole $900 \leqslant T_{\text {eff }} \leqslant 2800 \mathrm{~K}$ model range examined. Freytag et al. (2010) also find that the effective diffusion coefficients in the stratosphere, where convectively excited gravity waves are responsible for atmospheric mixing, also increase with increasing $T_{\text {eff }}$ for $T_{\text {eff }} \leqslant 1500 \mathrm{~K}$ and $T_{\text {eff }} \geqslant 2000 \mathrm{~K}$, but the behavior at intermediate $1500<T_{\text {eff }}<2000 \mathrm{~K}$ becomes more complicated due to the effects of clouds, which alter atmospheric stability. At the base of the stratosphere in the Freytag et al. (2010) models, the effective diffusion coefficient goes through a minimum. The $K_{z z}$ profiles are also sensitive to gravity and the overall static stability in the atmosphere. Without running realistic dynamical models for the planets in question, we cannot reliably estimate $K_{z z}$ profiles a priori, and we caution that our empirical profiles may have different magnitudes or functional forms than those of the real youngJupiter atmospheres. In particular, our profiles do not have the very weak $K_{z z}$ minimum that might be expected at the base of the stratosphere on young Jupiters. Because this minimum $K_{z z}$ results in maximum column abundances for photochemical species produced at high altitudes (e.g., Bézard et al. 2002), our convention may cause us to underestimate the abundances of photochemical products, but not as severely as if we assumed that $K_{z z}$ were constant throughout the atmosphere.

The photochemical model results also depend on the host star's ultraviolet flux and spectral energy distribution (e.g., Venot et al. 2013; Miguel \& Kaltenegger 2014; Miguel et al. 2015). For our specific exoplanet models, both 51 Eri (spectral type F0) and HR 8799 (spectral type A5) are expected to be brighter than the Sun at UV wavelengths (see Figure 3). However, the only direct ultraviolet spectral observations we could find for either star are derived from International Ultraviolet Explorer (IUE) satellite observations of 51 Eri in the MAST archive (http://archive.stsci.edu). Therefore, except for these IUE observations, our assumed stellar spectra are assembled from a variety of theoretical sources. For wavelengths greater than $1979 \AA$, the 51 Eri spectrum is taken from the Heap \& Lindler (2011) NextGen model for 51 Eri (HD 29391); for wavelengths between 1200 and $1978.72 \AA$ A-except right at $\mathrm{H} \mathrm{Ly} \alpha$-we use IUE observations of 51 Eri from the MAST IUE archive; for wavelengths less than $\sim 1150 \AA$, we 

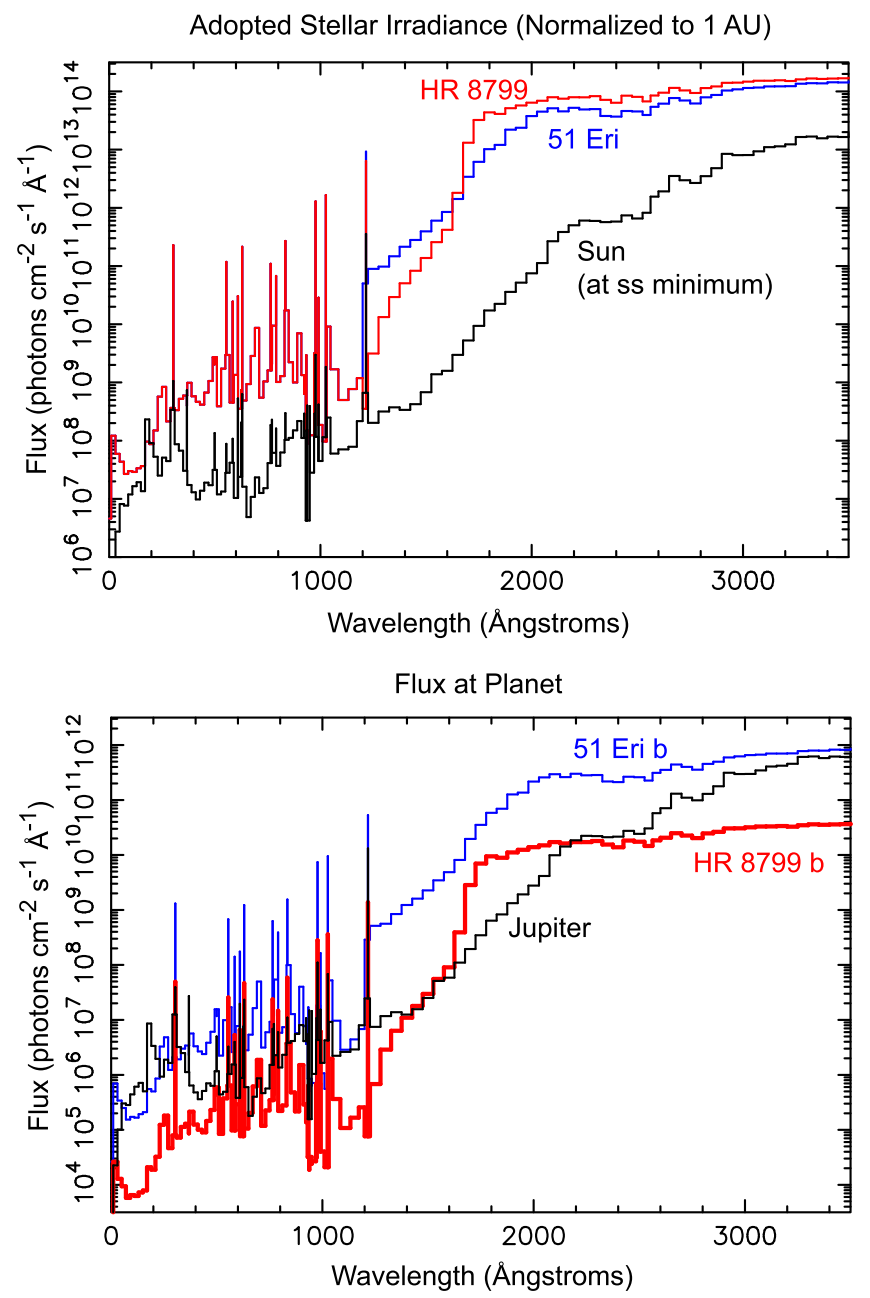

Figure 3. Ultraviolet stellar irradiance adopted in the models. (Top) The irradiance of 51 Eri (blue) and HR 8799 (red) as received at 1 au, in comparison with that the Sun (black); (Bottom) the irradiance at the top of the planet's atmosphere for 51 Eri b (blue) and HR 8799 b (red) in comparison with Jupiter (black). Note from the top panel that both 51 Eri and HR 8799 are brighter than the Sun in the ultraviolet, but 51 Eri b and HR 8799 b are farther away from their host stars than Jupiter, so in terms of the H Ly $\alpha$ flux received, which drives much of the interesting photochemistry, Jupiter receives a flux intermediate between 51 Eri b and HR 8799 b (bottom panel).

adopt the theoretical spectrum of HR 8799 (as the closest analog star) from the Sanz-Forcada et al. (2011) X-exoplanets archive; and for Ly $\alpha$ at $1215.7 \AA$, we adopt the reconstructed intrinsic H Ly $\alpha$ flux for 51 Eri from Landsman \& Simon (1993). The HR 8799 spectrum is a composite of several theoretical models. At wavelengths less than $1150 \AA$ and in the wavelength bin at $1190 \mathrm{~A}$, the HR 8799 spectrum is from the aforementioned Sanz-Forcada et al. (2011) model of HR 8799; at wavelengths greater than $1150 \AA$ - except for the wavelength bins at 1190 and $1215.7 \AA$-we use a Castelli \& Kurucz (2004) model with assumed parameters of $T_{\text {eff }}=7500 \mathrm{~K}, \log (g)=4.5$ (cgs), $\log [\mathrm{Fe} / \mathrm{H}]=-0.5$, radius $=1.44 R_{\odot}$; and for $1215.7 \AA$, we estimate the flux as the average of four stars $\left(\kappa^{2}\right.$ Tau [A7V], HR 1507 [F0V], 30 LMi [F0V], $\alpha$ Hyi [F0V]) from the Landsman \& Simon (1993) database of reconstructed intrinsic H Ly $\alpha$ fluxes, after scaling appropriately for stellar distance. For the spectral irradiance of the Sun shown in Figure 3, we adopt the solar-cycle minimum spectrum of Woods \& Rottman (2002).
Note from Figure 3 that 51 Eri and HR 8799 are intrinsically brighter than the Sun in the ultraviolet. Despite the great orbital distances of the HR 8799 planets (b at $\sim 68$ au, c at $\sim 43$ au, d at $\sim 27$ au; cf. Marois et al. 2008 \& Maire et al. 2015) and 51 Eri b (14 au according to De Rosa et al. 2015, although we used $13.2 \mathrm{au}$ for the calculations based on the earlier report by Macintosh et al. 2015), these planets-like the giant planets within our own solar system-receive sufficient ultraviolet flux that photochemistry should be effective. In fact, 51 Eri b receives a greater $\mathrm{H}$ Ly $\alpha$ flux than any of our solar-system giant planets, including Jupiter (see Figure 3), while the most distant HR 8799 b receives a greater H Ly $\alpha$ flux than either Uranus or Neptune, which both have rich stratospheric hydrocarbon photochemistry (Summers \& Strobel 1989; Romani et al. 1993; Moses et al. 1995; Dobrijevic et al. 2010; Orton et al. 2014). Indeed, the first investigation into the photochemistry of 51 Eri b (Zahnle et al. 2016) suggests that photochemical production of complex hydrocarbons and sulfur species will be important on this young Jupiter and may lead to the formation of sulfur and hydrocarbon hazes.

\section{RESULTS}

Results from our thermo/photochemical kinetics and transport model are presented below. We first discuss the results for generic directly imaged planets, including trends as a function of $T_{\text {eff }}, \log (g), K_{\text {deep }}$, and distance from the host star (see also Zahnle \& Marley 2014). The relevant disequilibrium chemistry that could potentially affect the spectral appearance of young Jupiters is described. Then, we present specific models for HR $8799 \mathrm{~b}$ and 51 Eri b and compare to observations. Note that the model abundance profiles for both the generic and specific planets discussed below are available as supporting material.

\subsection{Generic Directly Imaged Planets: Chemistry}

For our "generic" young Jupiters, we generate a suite of models for nine different effective temperatures ( $T_{\text {eff }}$ ranging from $600 \mathrm{~K}$ to $1400 \mathrm{~K}$, at $100 \mathrm{~K}$ intervals), seven different eddy diffusion coefficient profiles (see Figure 2), and two different surface gravities $\left(g=10^{3.5}\right.$ and $\left.10^{4} \mathrm{~cm} \mathrm{~s}^{-2}\right)$. The thermal profiles of these models are shown in Figure 1. Note from Figure 1 that all the models have deep atmospheres that lie within the $\mathrm{CO}$ stability field, whereas all but the hottest models switch over to the $\mathrm{CH}_{4}$ stability field in the upper atmosphere. Therefore, if the atmosphere were to remain in chemical equilibrium, $\mathrm{CH}_{4}$ would be the dominant carbon constituent at "photospheric" pressures in the $10^{3}-0.1$ mbar range for most of these planets, and methane absorption would be prominent in the near-infrared emission spectra. However, $\mathrm{CO} \leftrightarrows \mathrm{CH}_{4}$ chemical equilibrium cannot be maintained at temperatures $\lesssim 1300 \mathrm{~K}$ for any reasonable assumption about the eddy diffusion coefficient profile (e.g., Visscher \& Moses 2011), and quenching will occur in the deep, convective regions of these planets. For all the thermal profiles investigated, the $\mathrm{CO}-\mathrm{CH}_{4}$ quench point occurs within the $\mathrm{CO}$ stability field, and the quenched abundance of $\mathrm{CO}$ will be greater than that of $\mathrm{CH}_{4}$. 

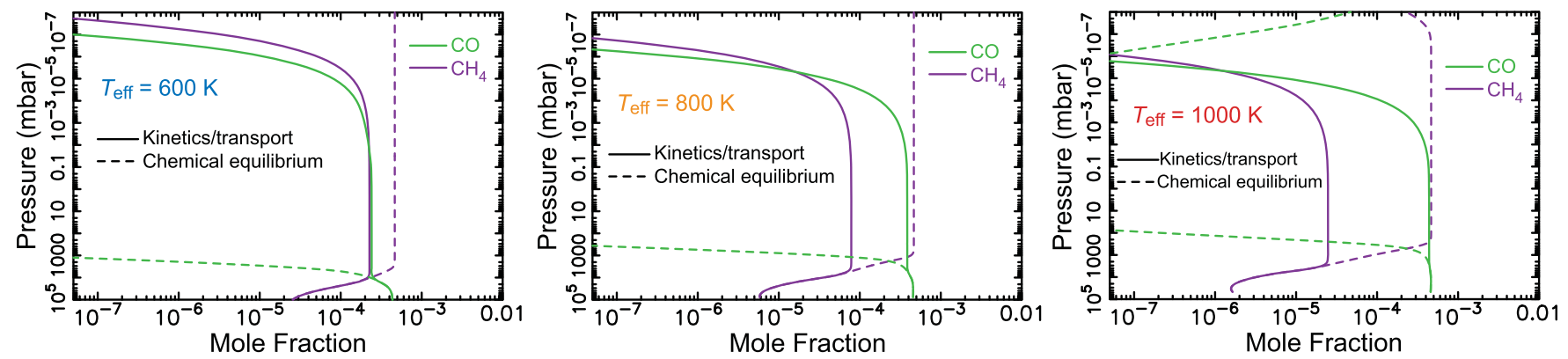

Figure 4. The vertical mixing-ratio profiles of $\mathrm{CH}_{4}$ (purple) and $\mathrm{CO}$ (green) for planets with a surface gravity $\log (\mathrm{g})=4$ (cgs), a moderate eddy mixing $K_{\text {deep }}=10^{7}$ $\mathrm{cm}^{2} \mathrm{~s}^{-1}$, and $T_{\text {eff }}=600 \mathrm{~K}$ (Left), $800 \mathrm{~K}$ (Middle), $1000 \mathrm{~K}$ (Right). Results for chemical equilibrium are shown with dashed lines, and results from our thermo/ photochemical kinetics and transport model are shown as solid lines. Note that $\mathrm{CH}_{4}$ dominates in the observable portion of the atmosphere in chemical equilibrium, whereas $\mathrm{CO}$ dominates in the disequilibrium models. The $\mathrm{CH}_{4} / \mathrm{CO}$ ratio is strongly dependent on temperature for both types of chemistry, with a higher $\mathrm{CH}_{4} / \mathrm{CO}$ ratio being favored for cooler planets.

The dominant kinetic reaction scheme converting $\mathrm{CO}$ to $\mathrm{CH}_{4}$ near the quench point in our models is

$$
\begin{aligned}
\mathrm{H}+\mathrm{CO}+\mathrm{M} & \rightarrow \mathrm{HCO}+\mathrm{M} \\
\mathrm{H}_{2}+\mathrm{HCO} & \rightarrow \mathrm{H}_{2} \mathrm{CO}+\mathrm{H} \\
\mathrm{H}+\mathrm{H}_{2} \mathrm{CO}+\mathrm{M} & \rightarrow \mathrm{CH}_{2} \mathrm{OH}+\mathrm{M} \\
\mathrm{H}_{2}+\mathrm{CH}_{2} \mathrm{OH} & \rightarrow \mathrm{CH}_{3} \mathrm{OH}+\mathrm{H} \\
\mathrm{CH}_{3} \mathrm{OH}+\mathrm{M} & \rightarrow \mathrm{CH}_{3}+\mathrm{OH}+\mathrm{M} \\
\mathrm{H}_{2}+\mathrm{CH}_{3} & \rightarrow \mathrm{CH}_{4}+\mathrm{H} \\
\mathrm{H}_{2}+\mathrm{OH} & \rightarrow \mathrm{H}_{2} \mathrm{O}+\mathrm{H} \\
2 \mathrm{H}+\mathrm{M} & \rightarrow \mathrm{H}_{2}+\mathrm{M} \\
\hline \text { Net: } \mathrm{CO}+3 \mathrm{H}_{2} & \rightarrow \mathrm{CH}_{4}+\mathrm{H}_{2} \mathrm{O},
\end{aligned}
$$

with $\mathrm{M}$ representing any third atmospheric molecule or atom. This scheme is identical to the dominant scheme (15) that Visscher \& Moses (2011) propose is controlling the conversion of $\mathrm{CO}$ into $\mathrm{CH}_{4}$ on brown dwarfs and is just the reverse of the scheme (3) that Moses et al. (2011) propose is controlling $\mathrm{CH}_{4}$ $\rightarrow \mathrm{CO}$ quenching on hot Jupiters. The rate-limiting step in the above scheme is the reaction $\mathrm{CH}_{3} \mathrm{OH}+\mathrm{M} \rightarrow \mathrm{CH}_{3}+\mathrm{OH}+\mathrm{M}$, where the rate coefficient is derived from the reverse reaction from Jasper et al. (2007). Our chemical model differs from some others in the literature (e.g., Venot et al. 2012; Zahnle \& Marley 2014) in that we adopt a slower rate coefficient for $\mathrm{H}+$ $\mathrm{CH}_{3} \mathrm{OH} \rightarrow \mathrm{H}_{2} \mathrm{O}+\mathrm{CH}_{3}$ based on the ab initio transition-state theory calculations of Moses et al. (2011) \& Lendvay et al. (1997), and the discussion of relevant experimental data in Norton \& Dryer (1990). However, the rate coefficient for this reaction adopted by Zahnle \& Marley (2014) and Zahnle et al. (2016) is slow enough that $\mathrm{CH}_{3} \mathrm{OH}+\mathrm{M} \rightarrow \mathrm{CH}_{3}+\mathrm{OH}+\mathrm{M}$ is usually faster, and hence their quench results are not greatly different from those described here. In any case, quenching is very effective in all the generic young-Jupiter models we investigated, and $\mathrm{CO}$ replaces $\mathrm{CH}_{4}$ as the dominant carbon species in the photospheres of these planets.

\subsection{1. $\mathrm{CO}-\mathrm{CH}_{4}$ Quenching as a Function of $T_{\text {eff }}$ and $K_{z z}$}

Figure 4 shows how the methane and carbon monoxide abundance vary with the planet's effective temperature (for $T_{\text {eff }}=600,800,1000 \mathrm{~K}$ ), for both the assumption of chemical equilibrium and from our thermo/photochemical kinetics and transport modeling, for $K_{\text {deep }}=10^{7} \mathrm{~cm}^{2} \mathrm{~s}^{-1}$ and $\log (g)=4$ (cgs). Figure 4 emphasizes just how significantly thermochemical equilibrium fails in its predictions for the composition of directly imaged planets, underpredicting the $\mathrm{CO}$ abundance by many orders of magnitude, and overpredicting the $\mathrm{CH}_{4}$ abundance. The $\mathrm{CO}-\mathrm{CH}_{4}$ quench point is discernible in the plot-it is the pressure at which the $\mathrm{CH}_{4}$ and $\mathrm{CO}$ mixing ratios stop following the equilibrium profiles and become constant with altitude. For the $T_{\text {eff }}=600 \mathrm{~K}$ planet, the quench point is near the $\mathrm{CO}=\mathrm{CH}_{4}$ equal-abundance curve shown in Figure 1, and carbon monoxide and methane quench at nearly equal abundances. Warmer planets have quench points more solidly within the $\mathrm{CO}$ stability field, and so the $\mathrm{CO}$ abundance then exceeds that of methane at high altitudes. The quenched $\mathrm{CH}_{4}$ abundance depends strongly on $T_{\text {eff }}$, decreasing with increasing $T_{\text {eff }}$, when other factors like $K_{z z}$ and $g$ are kept identical. The depletion in both the $\mathrm{CO}$ and $\mathrm{CH}_{4}$ mixing ratios at high altitudes in the disequilibrium models in Figure 4 is due to molecular diffusion, which is dependent on temperature. Planets with a higher $T_{\text {eff }}$ have warmer upper atmospheres, causing molecular diffusion to take over at deeper levels. Therefore, warmer planets have homopause levels at higher pressures (lower altitudes), all other things being equal.

The quenched species abundances also depend strongly on $K_{\text {deep }}$ and on surface gravity. Figure 5 illustrates this relationship for $\mathrm{CO}$ (top row) and $\mathrm{CH}_{4}$ (bottom row) for a suite of generic young Jupiter models, with the lower-gravity (log $(g)=3.5)$ case being plotted in the left column and the highergravity case $(\log (g)=4.0)$ in the right column. Note from Figure 5 that the quenched $\mathrm{CH}_{4}$ abundance is highly sensitive to both $T_{\text {eff }}$ and $K_{\text {deep }}$, and is greatest for low temperatures and weak deep vertical mixing. Higher-gravity planets with the same $T_{\text {eff }}$ are cooler at any particular pressure level, so higher $g$ favors increased $\mathrm{CH}_{4}$ abundance, all other factors being equal. In contrast, high $g$, low $T_{\text {eff }}$, and low $K_{\text {deep }}$ favor smaller quenched $\mathrm{CO}$ abundances. Note, however, the nearly constant quenched $\mathrm{CO}$ mixing ratio over a large swath of parameter space in Figure 5 for these two relatively low surface gravities. The quenched $\mathrm{CO}$ mixing ratio is less sensitive than $\mathrm{CH}_{4}$ to variations in $T_{\text {eff }}, K_{\text {deep }}$, and $g$ in this range because $\mathrm{CO}$ is dominant at the quench point, and the equilibrium $\mathrm{CO}$ mixing ratio is more constant with height through the quench region, whereas the equilibrium $\mathrm{CH}_{4}$ mixing-ratio profile in this region has a significant vertical gradient.

This is an important point. Disequilibrium chemistry from transport-induced quenching will cause $\mathrm{CO}-$ not $\mathrm{CH}_{4}$ - to dominate in the photospheres of virtually all directly imaged young planetary-mass (and planetary-gravity) companions, 

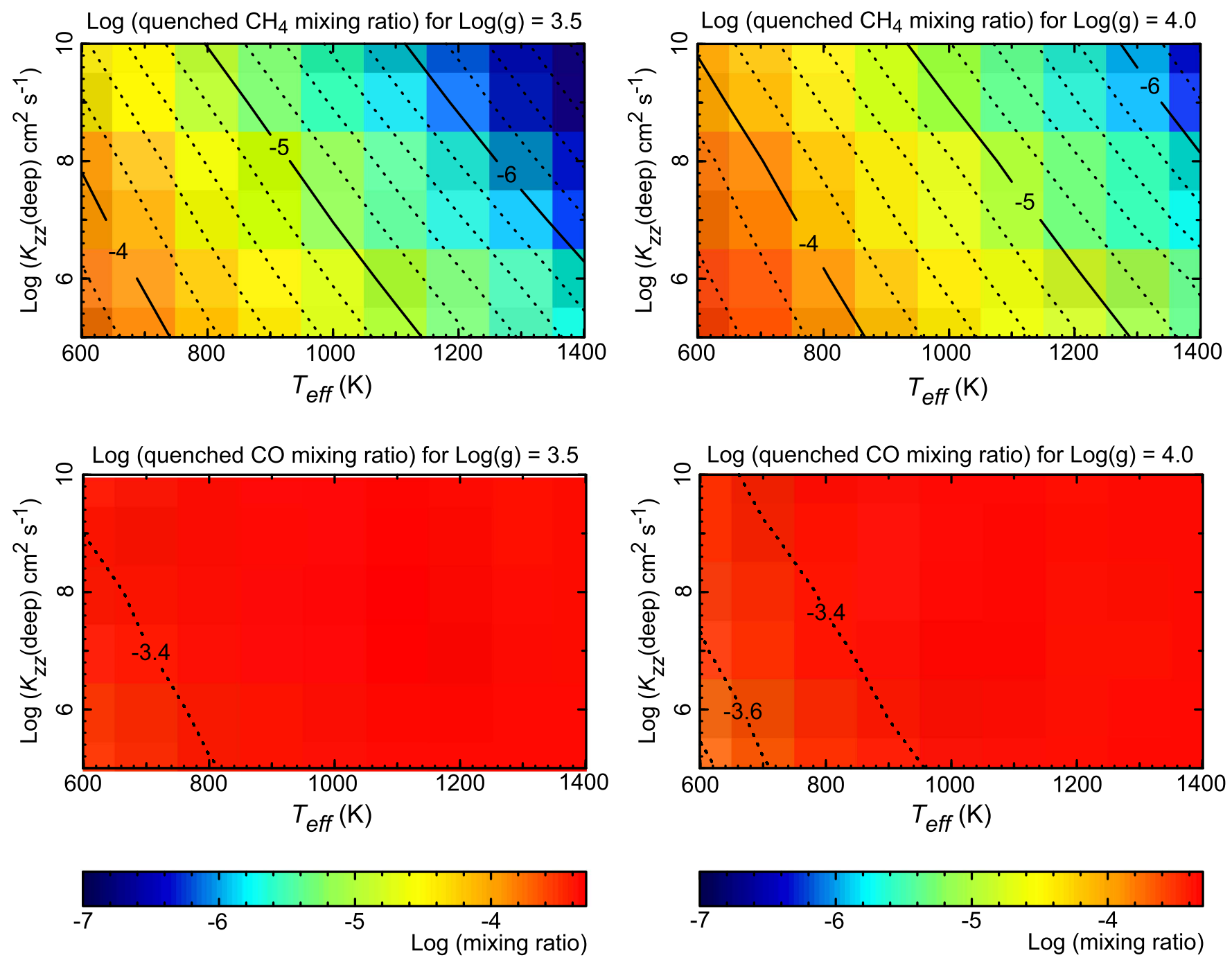

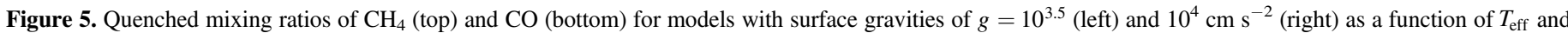

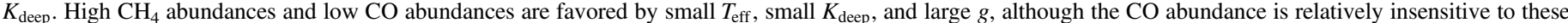
factors over the range of models investigated.

despite the equilibrium predictions for the predominance of $\mathrm{CH}_{4}$; in addition, the $\mathrm{CO}$ abundance should be similar for directly imaged planets with the same metallicity. Spectral signatures of $\mathrm{CO}$ should therefore be common for young Jupiters, and derived $\mathrm{CO}$ abundances can help constrain the planet's metallicity. Note that this conclusion changes for higher-gravity ( $g \gtrsim 10^{5} \mathrm{~cm} \mathrm{~s}^{-2}$ ) T dwarfs in this temperature range (Hubeny \& Burrows 2007; Zahnle \& Marley 2014), where $\mathrm{CH}_{4}$ can dominate and $\mathrm{CO}$ is the minor species.

\subsubsection{Sensitivity of Disequilibrium Chemistry to $K_{z z}$}

Figure 6 illustrates how the abundances of several constituents change with the different eddy diffusion coefficient profiles shown in Figure 2, for a model with $T_{\text {eff }}=1000$ $\mathrm{K}, \log (g)=4.0$, and an orbital distance of 68 au from a star with the properties of HR 8799. As the eddy diffusion coefficient at depth, $K_{\text {deep }}$, is increased, vertical transport begins to dominate at greater and greater depths over the chemical kinetic reactions that act to maintain equilibrium. Smaller $K_{\text {deep }}$ values lead to mixing ratio profiles that follow the equilibrium profiles to higher altitudes before quenching occurs. The quenched methane abundance therefore increases with decreasing $K_{\text {deep }}$, and species that are produced through the photochemical destruction of methane, like $\mathrm{C}_{2} \mathrm{H}_{2}$ and $\mathrm{C}_{2} \mathrm{H}_{6}$, also have mixing ratios that increase with decreasing $K_{\text {deep }}$. Conversely, the quenched $\mathrm{CO}$ abundance decreases with decreasing $K_{\text {deep }}$, but because the chemical equilibrium abundance of $\mathrm{CO}$ is only slightly decreasing with altitude over the range of quench points for the different $K_{\text {deep }}$ values investigated, the quenched $\mathrm{CO}$ mixing ratio is relatively insensitive to $K_{\text {deep }}$.

Water quenches via reaction scheme (1) above at the same point as that of $\mathrm{CO}$ and $\mathrm{CH}_{4}$. Since the equilibrium mixing ratio for $\mathrm{H}_{2} \mathrm{O}$ is increasing with increasing altitude very slightly over the pressure range of the quench points, the quenched $\mathrm{H}_{2} \mathrm{O}$ abundance very slightly increases with decreasing $K_{\text {deep }}$. Water is a key opacity source in young Jupiters that affects how efficiently heat is lost from the planet, so it is important to keep in mind that the resulting quenched water mixing ratio on directly imaged planets can be a factor of a few below that of chemical-equilibrium predictions in the photosphere. This quenching of $\mathrm{H}_{2} \mathrm{O}$ becomes more important for higher $T_{\mathrm{eff}}$, larger $K_{\text {deep }}$, and lower surface gravities. Quenching of water 

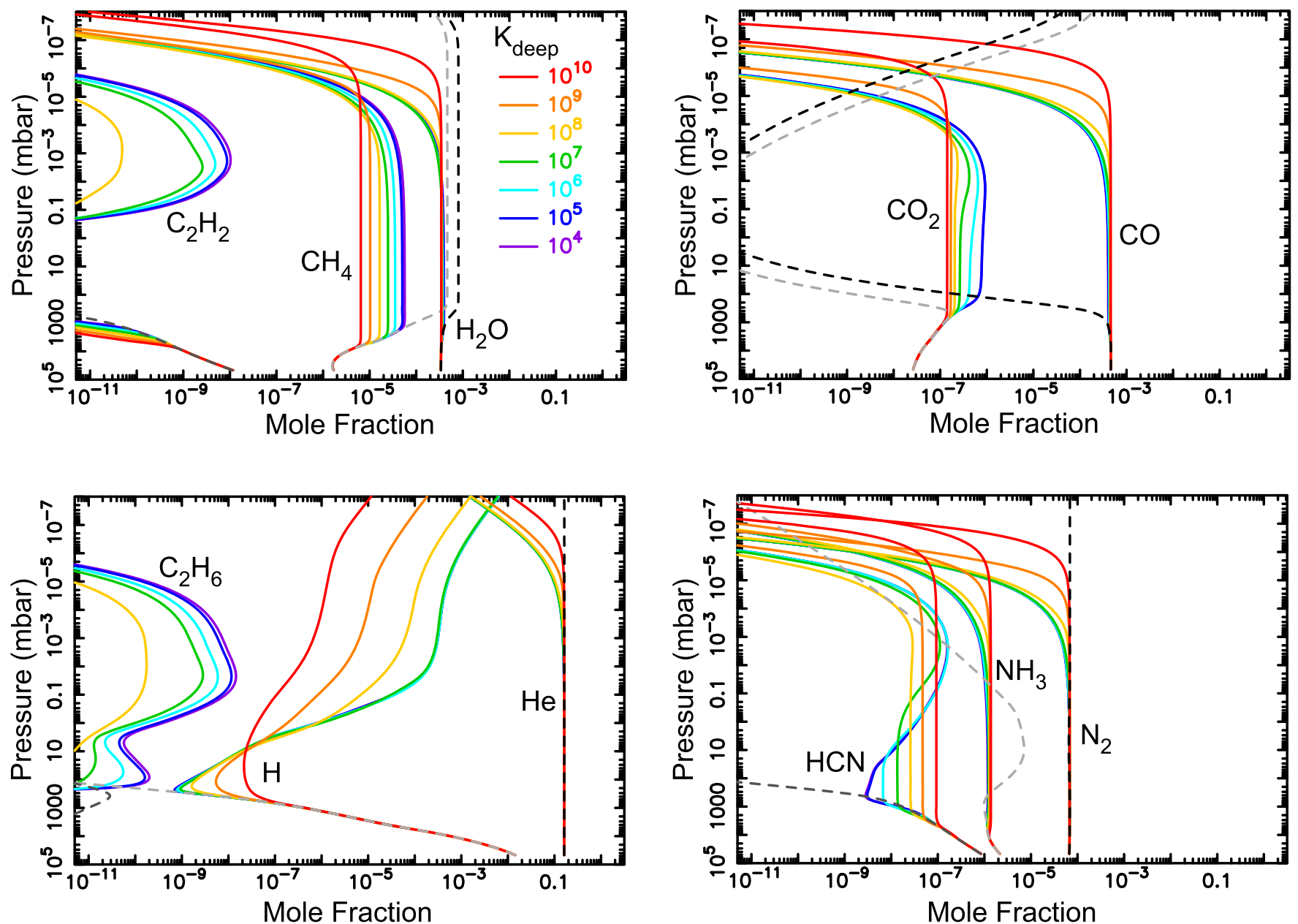

Figure 6. Vertical profiles of several important species in our thermo/photochemical kinetics and transport models (solid colored lines) and in chemical equilibrium (dashed gray and black lines) for a planet with $T_{\text {eff }}=1000 \mathrm{~K}$ and $g=10^{4} \mathrm{~cm} \mathrm{~s}^{-2}$, at a distance of 68 au from a star with properties like HR 8799 (Figure 3 ), as a function of $K_{\text {deep }}$ (see the legend in the top left panel, and the $K_{z z}$ profiles shown in Figure 2). Note that the atmosphere is far out of equilibrium for all the eddy diffusion coefficient profiles considered. The quenched $\mathrm{CH}_{4}$ mixing ratio increases with decreasing $K_{\text {deep }}$. The mixing ratios of methane photochemical products such as $\mathrm{C}_{2} \mathrm{H}_{2}, \mathrm{C}_{2} \mathrm{H}_{6}$, and $\mathrm{H}$ also increase with decreasing $K_{\text {deep }}$. Water quenches at the same time as $\mathrm{CO}$ and $\mathrm{CH}_{4}$, remaining in disequilibrium in the photosphere. Species like $\mathrm{HCN}$ and $\mathrm{CO}_{2}$ are affected both by photochemistry and by quenching of the major carbon, oxygen, and nitrogen species.

should therefore be considered in models that calculate the thermal evolution of brown dwarfs and directly imaged planets, particularly for young, small, hot objects.

The $\mathrm{NH}_{3}-\mathrm{N}_{2}$ quench point is deeper than that of $\mathrm{CO}-\mathrm{CH}_{4-}$ $\mathrm{H}_{2} \mathrm{O}$. For all the planets considered, this major nitrogen-species quench point is well within the $\mathrm{N}_{2}$-dominated regime, so $\mathrm{N}_{2}$ dominates in the photosphere, and $\mathrm{NH}_{3}$ is less abundant. The equilibrium profiles are not strongly sloped in the quench region, so the quenched abundances of $\mathrm{NH}_{3}$-and $\mathrm{N}_{2}$ in particular-are not very sensitive to $K_{\text {deep }}$ (see Figure 6). The dominant quenching scheme for $\mathrm{N}_{2} \rightarrow \mathrm{NH}_{3}$ in our generic young-Jupiter models is

$$
\begin{aligned}
\mathrm{H}+\mathrm{N}_{2}+\mathrm{M} & \rightarrow \mathrm{N}_{2} \mathrm{H}+\mathrm{M} \\
\mathrm{H}_{2}+\mathrm{N}_{2} \mathrm{H} & \rightarrow \mathrm{N}_{2} \mathrm{H}_{2}+\mathrm{H} \\
\mathrm{H}+\mathrm{N}_{2} \mathrm{H}_{2} & \rightarrow \mathrm{NH}+\mathrm{NH}_{2} \\
\mathrm{H}_{2}+\mathrm{NH} & \rightarrow \mathrm{NH}_{2}+\mathrm{H} \\
2\left(\mathrm{H}_{2}+\mathrm{NH}_{2}\right. & \left.\rightarrow \mathrm{NH}_{3}+\mathrm{H}\right) \\
2 \mathrm{H}+\mathrm{M} & \rightarrow \mathrm{H}_{2}+\mathrm{M} \\
\hline \text { Net: } \mathrm{N}_{2}+3 \mathrm{H}_{2} & \rightarrow 2 \mathrm{NH}_{3},
\end{aligned}
$$

which is simply the reverse of reaction scheme (5) for $\mathrm{NH}_{3} \rightarrow$ $\mathrm{N}_{2}$ quenching discussed in Moses et al. (2011). The ratelimiting step in the above scheme is the reaction $\mathrm{H}+\mathrm{N}_{2} \mathrm{H}_{2} \rightarrow$ $\mathrm{NH}+\mathrm{NH}_{2}$, where the rate coefficient derives from the reverse reaction, as determined by Klippenstein et al. (2009).

Constituents such as $\mathrm{HCN}$ and $\mathrm{CO}_{2}$ are affected both by photochemistry and by quenching of the dominant carbon, nitrogen, and oxygen carriers $\left(\mathrm{H}_{2} \mathrm{O}, \mathrm{CO}, \mathrm{CH}_{4}, \mathrm{NH}_{3}\right.$, and $\left.\mathrm{N}_{2}\right)$ and thus exhibit complicated vertical profiles in Figure 6. For large values of $K_{z z}(z)$, transport controls the $\mathrm{HCN}$ and $\mathrm{CO}_{2}$ profiles throughout the atmospheric column. The quenched abundance of HCN increases with increasing $K_{\text {deep }}$ because the equilibrium profile decreases with height within the quench region. Conversely, the quenched abundance of $\mathrm{CO}_{2}$ decreases with increasing $K_{\text {deep }}$ because the equilibrium profile increases with height near the quench point; moreover, the photochemically produced $\mathrm{CO}_{2}$ takes longer to diffuse downward when the stratospheric $K_{z z}$ is smaller, so a larger column abundance can build up. In fact, at higher altitudes with the smaller $K_{\text {deep }}$ models, photochemical production of $\mathrm{HCN}$ and $\mathrm{CO}_{2}$ can dominate over transport from below, and the resulting mixingratio "bulges" in the stratosphere represent the signatures of 
that photochemical production. In general, the columnintegrated $\mathrm{CO}_{2}$ abundance increases with decreasing $K_{\text {deep }}$, while that of $\mathrm{HCN}$ decreases with decreasing $K_{\text {deep }}$. However, this latter result also depends on the planet's thermal structure and incident ultraviolet flux.

Note that the sharp drop off in the species profiles at high altitudes in Figure 6 is due to molecular diffusion. Because the molecular diffusion coefficient profiles for this thermal structure cross the $K_{z z}$ profiles at relatively high altitudes where the $K_{z z}$ profiles have already transitioned to the $P^{0.5}$ sloped region, the homopause levels for most of the models for any particular species are the same for the different $K_{z z}$ models. However, the $\mathrm{CH}_{4}$ homopause level is at $\sim 3 \times 10^{-7} \mathrm{mbar}$ for the sloped $K_{z z}$ case, and Figure 2 shows that the $K_{\text {deep }}=10^{10}$ $\mathrm{cm}^{2} \mathrm{~s}^{-1} K_{z z}$ profile does not reached the sloped $K_{z z}$ portion until pressures less than a few $\times 10^{-8}$ mbar. Therefore, the $\mathrm{CH}_{4}$ molecular diffusion coefficient crosses the $K_{\text {deep }}=10^{10} \mathrm{~cm}^{2}$ $\mathrm{s}^{-1} K_{z z}$ profile at a higher altitude (lower pressure) than the other models, leading to a higher-altitude homopause and $\mathrm{CH}_{4}$ being carried to higher altitudes in that model than the others. Similarly, the $\mathrm{H}_{2} \mathrm{O}, \mathrm{NH}_{3}, \mathrm{CO}$, and $\mathrm{N}_{2}$ molecular diffusion coefficients cross the sloped $K_{z z}$ profile at pressures between where the $K_{\text {deep }}=10^{8}$ and $10^{9} \mathrm{~cm}^{2} \mathrm{~s}^{-1}$ models transition to the sloped case, so both the $K_{z z}=10^{9}$ and $10^{10} \mathrm{~cm}^{2} \mathrm{~s}^{-1}$ cases have higher-altitude $\mathrm{H}_{2} \mathrm{O}, \mathrm{NH}_{3}, \mathrm{CO}$, and $\mathrm{N}_{2}$ homopauses than the other models.

\subsubsection{Sensitivity of Disequilibrium Chemistry to Orbital Distance}

Figure 7 illustrates how the disequilibrium composition changes as a function of distance from the host star, for planets with $T_{\text {eff }}=1000 \mathrm{~K}, \log (g)=4.0(\mathrm{cgs}), K_{\text {deep }}=10^{7} \mathrm{~cm}^{2} \mathrm{~s}^{-1}$, orbiting at 10,32 , and 100 au from a star with the properties of HR 8799. Because the strong interior heat dominates the energy transport on these young planets, the thermal structures are virtually identical in these cases, so the main differences in the models are due to the incoming ultraviolet flux. The closer a planet is to its star, the greater the UV irradiation received, leading to greater destruction rates of key molecules such as $\mathrm{CH}_{4}, \mathrm{NH}_{3}, \mathrm{H}_{2} \mathrm{O}, \mathrm{CO}$, and $\mathrm{N}_{2}$. That in turn leads to greater production rates of photochemical products such as $\mathrm{HCN}, \mathrm{CO}_{2}$, $\mathrm{C}_{2} \mathrm{H}_{2}, \mathrm{C}_{2} \mathrm{H}_{6}$, complex hydrocarbons such as methylacetylene (an isomer of $\mathrm{C}_{3} \mathrm{H}_{4}$ ) and benzene (an isomer of $\mathrm{C}_{6} \mathrm{H}_{6}$ ), complex nitriles such as $\mathrm{HC}_{3} \mathrm{~N}$, small oxygen-bearing species such as $\mathrm{NO}$ and $\mathrm{O}_{2}$, and small radicals and atoms such as $\mathrm{C}, \mathrm{N} . \mathrm{O}, \mathrm{OH}$, $\mathrm{NH}_{2}$, and $\mathrm{CH}_{3}$.

The dominant photochemical product on young Jupiters is atomic hydrogen. The atomic $\mathrm{H}$ is derived largely from water photolysis (producing $\mathrm{OH}+\mathrm{H}$ ), and the subsequent reaction of $\mathrm{OH}+\mathrm{H}_{2} \rightarrow \mathrm{H}_{2} \mathrm{O}+\mathrm{H}-\mathrm{a}$ two-step process that catalytically destroys $\mathrm{H}_{2}$ to produce two $\mathrm{H}$ atoms. In this regard, young Jupiters have more in common with close-in transiting giant planets (e.g., Liang et al. 2003) than our solar-system giant planets, and the copious amount of atomic $\mathrm{H}$ produced from this photochemistry (see Figure 7) affects much of the subsequent stratospheric chemistry on young Jupiters.

Another key photochemical product is $\mathrm{CO}_{2}$. Carbon dioxide is produced overwhelmingly from the reaction $\mathrm{OH}+\mathrm{CO} \rightarrow$ $\mathrm{CO}_{2}+\mathrm{H}$, with the $\mathrm{OH}$ deriving from water photolysis. If the stratosphere is relatively warm, as in the example shown in Figure 7 (with a $1 \mu$ bar temperature of $377 \mathrm{~K}$ ), the $\mathrm{OH}+\mathrm{H}_{2} \rightarrow$ $\mathrm{H}_{2} \mathrm{O}+\mathrm{H}$ reaction occurs at a much faster rate than $\mathrm{OH}+\mathrm{CO}$ $\rightarrow \mathrm{CO}_{2}+\mathrm{H}$, but the latter reaction provides a slow but steady
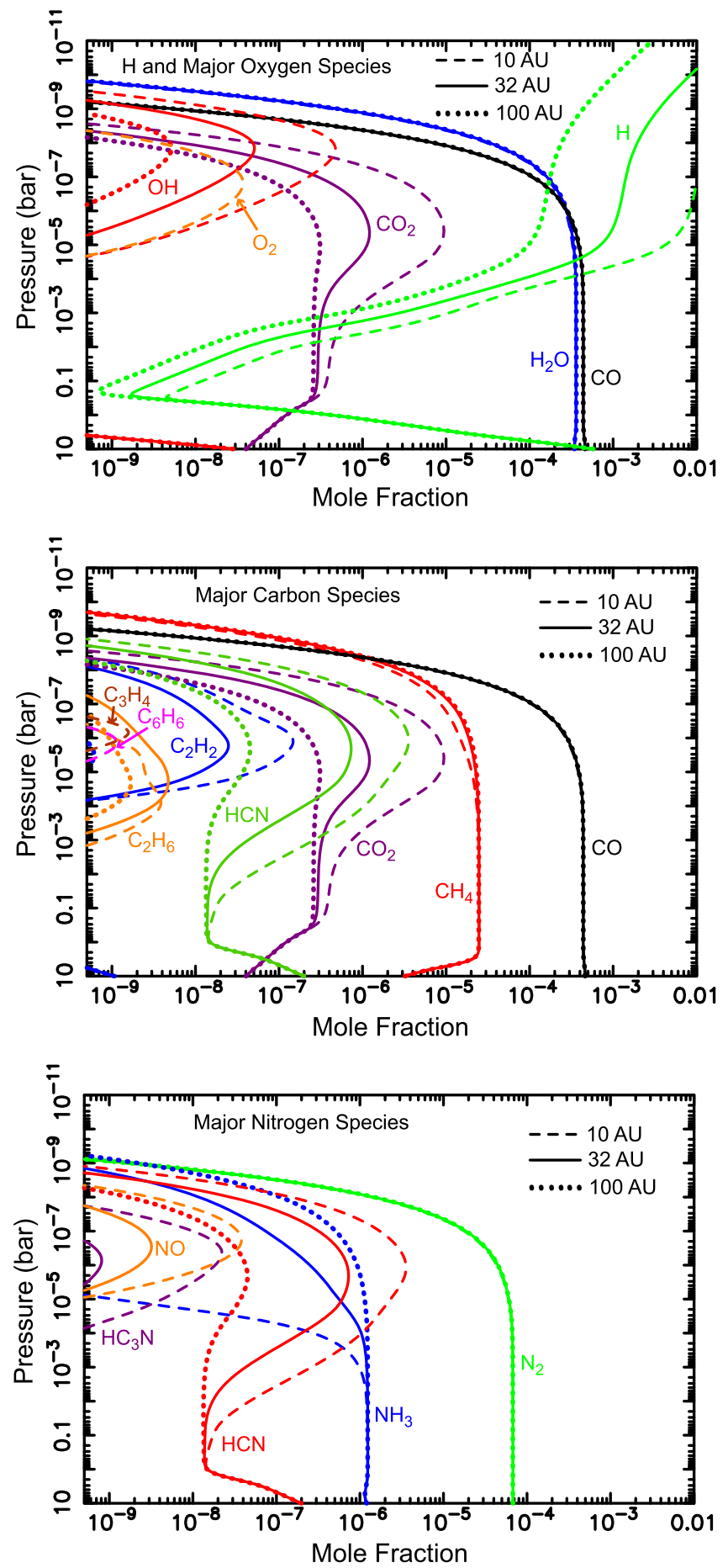

Figure 7. Vertical mixing-ratio profiles of several atmospheric species as a function of orbital distance for a planet with $T_{\text {eff }}=1000 \mathrm{~K}, g=10^{4} \mathrm{~cm} \mathrm{~s}^{-2}$, and $K_{\text {deep }}=10^{7} \mathrm{~cm}^{2} \mathrm{~s}^{-1}$, that is being irradiated by an HR 8799-like star at a distance of 10 au (dashed lines), 32 au (solid lines), and 0 au (dotted lines). The greater UV flux received by the closest-in planet leads to increased destruction of photochemically active "parent" molecules such as $\mathrm{CH}_{4}, \mathrm{NH}_{3}, \mathrm{H}_{2} \mathrm{O}, \mathrm{CO}$, and $\mathrm{N}_{2}$, and increased production of photochemical "daughter" products such as $\mathrm{HCN}, \mathrm{CO}_{2}$, complex hydrocarbons, complex nitriles, and atomic species and small radicals.

stream of oxygen away from water and $\mathrm{CO}$ into $\mathrm{CO}_{2}$. Loss of $\mathrm{CO}_{2}$ occurs through the reverse of the main production reaction (i.e., $\mathrm{H}+\mathrm{CO}_{2} \rightarrow \mathrm{CO}+\mathrm{OH}$ ), provided that the upperatmospheric temperature is warm enough to overcome the 
substantial energy barrier for this reaction, as well as through photolysis, through reaction of atomic $\mathrm{N}$ to produce $\mathrm{NO}+\mathrm{CO}$, and through reaction of $\mathrm{CH}$ to produce $\mathrm{HCO}+\mathrm{CO}$. Note that all the main loss processes for $\mathrm{CO}_{2}$ end up recycling the $\mathrm{CO}$. For our generic young Jupiter models, the column-integrated $\mathrm{CO}_{2}$ production rate exceeds the loss rate, and the photochemically produced $\mathrm{CO}_{2}$ diffuses down through the atmosphere until it reaches higher-temperature regions where it can once again reach a chemical balance with $\mathrm{CO}$ and $\mathrm{H}_{2} \mathrm{O}$. The greater the incident ultraviolet flux, the greater the net photochemical production rate of $\mathrm{CO}_{2}$ (see Figure 7).

Molecular oxygen becomes a notable high-altitude photochemical product on more highly irradiated young Jupiters. It is produced as a byproduct of the water photochemistry, where photolysis of $\mathrm{H}_{2} \mathrm{O}$ produces $\mathrm{OH}+\mathrm{H}$ and $\mathrm{O}+2 \mathrm{H}$, and the $\mathrm{OH}$ and $\mathrm{O}$ react to form $\mathrm{O}_{2}+\mathrm{H}$. The $\mathrm{O}_{2}$ is lost through photolysis (which primarily leads back to $\mathrm{H}_{2} \mathrm{O}$ eventually) and through reactions with atomic carbon (which leads to $\mathrm{CO}$ ).

Some of the $\mathrm{CH}_{4}$ in the upper atmospheres of young Jupiters will be oxidized to produce $\mathrm{CO}$ and eventually $\mathrm{CO}_{2}$. In our generic young Jupiter models, this process occurs through schemes such as:

$$
\begin{aligned}
\mathrm{H}_{2} \mathrm{O}+h \nu & \rightarrow 2 \mathrm{H}+\mathrm{O} \\
\mathrm{H}+\mathrm{CH}_{4} & \rightarrow \mathrm{CH}_{3}+\mathrm{H}_{2} \\
\mathrm{O}+\mathrm{CH}_{3} & \rightarrow \mathrm{H}_{2} \mathrm{CO}+\mathrm{H} \\
\mathrm{H}_{2} \mathrm{CO}+\mathrm{H} & \rightarrow \mathrm{HCO}+\mathrm{H}_{2} \\
\mathrm{HCO}+\mathrm{H} & \rightarrow \mathrm{CO}+\mathrm{H}_{2} \\
\mathrm{CH}_{4}+\mathrm{H}_{2} & \rightarrow \mathrm{CO}+3 \mathrm{H}_{2},
\end{aligned}
$$

with $h \nu$ representing an ultraviolet photon. Methane oxidation schemes such as the one above are more effective the higher the incident stellar ultraviolet flux.

As on the giant planets in our own solar system (e.g., Strobel 1983; Atreya \& Romani 1985; Yung \& DeMore 1999; Moses et al. 2004; Fouchet et al. 2009), the reduced hydrocarbon photochemistry in the atmospheres of young Jupiters will be efficacious and complex. However, the overall column abundance of the hydrocarbon species produced by neutral photochemistry (as opposed to ion chemistry) on young Jupiters will typically be smaller than on our own giant planets, as a result of the greater stratospheric temperatures, greater stratospheric water abundance, and different dominant and/or competing kinetic reactions, including methane recycling and oxidation. The typically smaller $\mathrm{CH}_{4}$ mixing ratio on young Jupiters (due to quenching) also contributes to the differences, as does a potentially larger stratospheric eddy $K_{z z}$ coefficient (due to upwardly propagating atmospheric waves generated in the rapidly convecting deep atmospheres of young Jupiters), which allows the high-altitude hydrocarbon photochemical products to be transported more rapidly to the deeper, hightemperature regions, where they become unstable. However, the larger stratospheric temperatures and resulting decreased stability of the complex hydrocarbons play a larger role.

As an example, the column abundance of ethane $\left(\mathrm{C}_{2} \mathrm{H}_{6}\right)$ above 100 mbar on Saturn (Moses et al. 2015), which is $\sim 10$ au from the Sun, is five orders of magnitude larger than that of the generic 10 au young Jupiter shown in Figure 7 , despite the greater $\mathrm{H} \mathrm{Ly} \alpha$ and overall UV flux received by the $10 \mathrm{au}$ generic young Jupiter around its brighter star. The main source of the ethane is still the same on both planets-the three-body reaction $\mathrm{CH}_{3}+\mathrm{CH}_{3}+\mathrm{M} \rightarrow \mathrm{C}_{2} \mathrm{H}_{6}+\mathrm{M}-$ but the $\mathrm{CH}_{3}$ on the $10 \mathrm{au}$ young Jupiter goes back to recycle the $\mathrm{CH}_{4}$ more than $99.9 \%$ of the time, because the higher atmospheric temperatures lead to a more efficient reaction of $\mathrm{CH}_{3}$ with $\mathrm{H}_{2}$ to form $\mathrm{CH}_{4}+\mathrm{H}$. Still, the total stratospheric column production rate of $\mathrm{C}_{2} \mathrm{H}_{6}$ is larger on the 10 au young Jupiter than on Saturn due to the brightness of the star and the larger UV flux; however, $\mathrm{C}_{2} \mathrm{H}_{6}$ is also more readily destroyed on the warmer young Jupiter through $\mathrm{H}+\mathrm{C}_{2} \mathrm{H}_{6} \rightarrow \mathrm{C}_{2} \mathrm{H}_{5}+\mathrm{H}_{2}$, with a much larger percentage of the carbon ending up back in $\mathrm{CH}_{4}$ rather than in $\mathrm{C}_{2} \mathrm{H}_{x}$ and other higher-order hydrocarbons. On Saturn, the photochemically produced $\mathrm{C}_{2} \mathrm{H}_{6}$ is much more chemically stable in the colder stratosphere, so the net production rate minus loss rate is greater on Saturn than on the generic $10 \mathrm{au}$ young Jupiter. It is also interesting to note that the direct photolysis of $\mathrm{CH}_{4}$ on our warmer generic young Jupiters is less important to the production of complex hydrocarbons than the reaction of atomic $\mathrm{H}$ with $\mathrm{CH}_{4}$ to form $\mathrm{CH}_{3}+\mathrm{H}_{2}$, with the $\mathrm{H}$ deriving from $\mathrm{H}_{2} \mathrm{O}$ photolysis (see discussion above).

Acetylene $\left(\mathrm{C}_{2} \mathrm{H}_{2}\right)$ is also an important photochemical product on our 10 au generic young Jupiter shown in Figure 7 that is produced through reaction schemes such as the following that first go through $\mathrm{C}_{2} \mathrm{H}_{6}$ and $\mathrm{C}_{2} \mathrm{H}_{4}$ :

$$
\begin{aligned}
2\left(\mathrm{H}_{2} \mathrm{O}+h \nu\right. & \rightarrow \mathrm{OH}+\mathrm{H}) \\
2\left(\mathrm{OH}+\mathrm{H}_{2}\right. & \left.\rightarrow \mathrm{H}_{2} \mathrm{O}+\mathrm{H}\right) \\
2\left(\mathrm{H}+\mathrm{CH}_{4}\right. & \left.\rightarrow \mathrm{CH}_{3}+\mathrm{H}_{2}\right) \\
\mathrm{CH}_{3}+\mathrm{CH}_{3}+\mathrm{M} & \rightarrow \mathrm{C}_{2} \mathrm{H}_{6}+\mathrm{M} \\
\mathrm{H}+\mathrm{C}_{2} \mathrm{H}_{6} & \rightarrow \mathrm{C}_{2} \mathrm{H}_{5}+\mathrm{H}_{2} \\
\mathrm{C}_{2} \mathrm{H}_{5}+\mathrm{M} & \rightarrow \mathrm{C}_{2} \mathrm{H}_{4}+\mathrm{H}+\mathrm{M} \\
\mathrm{H}+\mathrm{C}_{2} \mathrm{H}_{4} & \rightarrow \mathrm{C}_{2} \mathrm{H}_{3}+\mathrm{H}_{2} \\
\mathrm{H}+\mathrm{C}_{2} \mathrm{H}_{3} & \rightarrow \mathrm{C}_{2} \mathrm{H}_{2}+\mathrm{H}_{2} \\
\hline \mathrm{Net}: 2 \mathrm{CH}_{4} & \rightarrow \mathrm{C}_{2} \mathrm{H}_{2}+3 \mathrm{H}_{2} .
\end{aligned}
$$

Acetylene is lost (a) through insertion reactions with atomic $\mathrm{C}$ and $\mathrm{CH}$ radicals to form $\mathrm{C}_{3} \mathrm{H}_{2}$ and $\mathrm{C}_{3} \mathrm{H}_{3}$, (b) through reactions with atomic $\mathrm{H}$ to form $\mathrm{C}_{2} \mathrm{H}_{3}$, with subsequent reactions leading to other $\mathrm{C}_{2} \mathrm{H}_{x}$ species and eventual methane recycling, and (c) by photolysis, which leads predominantly to recycling of the $\mathrm{C}_{2} \mathrm{H}_{2}$. As on transiting hot Jupiters (Moses et al. 2011), the atomic carbon from loss process (a) here derives both from photolysis of $\mathrm{CO}$ and from methane photodestruction to form $\mathrm{CH}_{3}, \mathrm{CH}_{2}$, and $\mathrm{CH}$, which can react with $\mathrm{H}$ to eventually form $\mathrm{C}$.

The relative efficiency of $\mathrm{C}_{3} \mathrm{H}_{2}$ and $\mathrm{C}_{3} \mathrm{H}_{3}$ production in some of our more highly irradiated young-Jupiter models (e.g., the $10 \mathrm{au}$ case) is interesting and suggests that complex carbon-rich species like polycyclic aromatic hydrocarbons (PAHs) could potentially form on some directly imaged planets, and might even lead to the condensation of organic hazes in these atmospheres, as enthusiastically advocated by Zahnle et al. (2009, 2016). However, in general, the efficiency of production of refractory organics from simple precursors like $\mathrm{C}_{2} \mathrm{H}_{2}, \mathrm{C}_{2} \mathrm{H}_{6}$, and $\mathrm{C}_{4} \mathrm{H}_{2}$ in an $\mathrm{H}_{2}$-dominated atmosphere seems to have been overestimated by Zahnle et al. (2009), Miller-Ricci Kempton et al. (2012), and Morley et al. (2013) — their arguments would suggest that Jupiter, Saturn, and Neptune should be completely enshrouded in optically thick stratospheric hydrocarbon hazes, 
yet that is not the case. Because of a lack of laboratory or theoretical kinetic information on reactions of $\mathrm{C}_{3} \mathrm{H}_{2}$ and $\mathrm{C}_{3} \mathrm{H}_{3}$ with other hydrocarbon radicals under relevant low-pressure, reducing conditions, the fate of these $\mathrm{C}_{3} \mathrm{H}_{x}$ species is not obvious (see also Moses et al. 2011; Hébrard et al. 2013). Three-body addition reactions of $\mathrm{C}_{3} \mathrm{H}_{2}$ and $\mathrm{C}_{3} \mathrm{H}_{3}$ with abundant ambient $\mathrm{H}$ atoms can lead to $\mathrm{C}_{3} \mathrm{H}_{3}$ and $\mathrm{C}_{3} \mathrm{H}_{4}$, respectively, and the $\mathrm{C}_{3} \mathrm{H}_{3}$ can react with $\mathrm{CH}_{3}$ to form $\mathrm{C}_{4} \mathrm{H}_{6}$ (Fahr \& Nayak 2000; Knyazev \& Slagle 2001) or self-react to form various $\mathrm{C}_{6} \mathrm{H}_{6}$ isomers (Atkinson \& Hudgens 1999; Fahr \& Nayak 2000), but these three-body reactions are not particularly effective at low pressures. Therefore, $\mathrm{C}_{3} \mathrm{H}_{2}$ and $\mathrm{C}_{3} \mathrm{H}_{3}$ build up to mixing ratios of a few $\times 10^{-8}$ at high altitudes in our 10 au young-Jupiter model. The comparatively large abundance of $\mathrm{C}_{3} \mathrm{H}_{2}$ and $\mathrm{C}_{3} \mathrm{H}_{3}$ radicals here is likely an artifact of having insufficient knowledge of other possible loss mechanisms for these species, and we make a plea for future laboratory experiments or theoretical modeling to rectify this situation.

Benzene $\left(\mathrm{C}_{6} \mathrm{H}_{6}\right)$ itself is produced in our models through $\mathrm{C}_{3} \mathrm{H}_{3}-\mathrm{C}_{3} \mathrm{H}_{3}$ recombination, which first goes through a linear $\mathrm{C}_{6} \mathrm{H}_{6}$ isomer before eventual production of benzene (Fahr \& Nayak 2000). The benzene mixing ratio reaches $1 \mathrm{ppb}$ in our $10 \mathrm{au}$ model (see Figure 7), but neither benzene nor any of the other relatively light hydrocarbons considered by our model become abundant enough to achieve saturation and condense. Similarly, the coupled carbon-nitrogen photochemistry in our model leads to non-trivial amounts of complex nitriles such as $\mathrm{HC}_{3} \mathrm{~N}$ being produced (see Figure 7), but again, these relatively light nitriles never reach saturation. Our neutral chemistry alone does not lead to hazes on these planets. However, we know from Titan that organic hazes can readily form from ion chemistry in a $\mathrm{N}_{2}$-dominated atmosphere (Imanaka \& Smith 2007; Vuitton et al. 2007; Waite et al. 2007; Hörst et al. 2012), and the presence of $>10 \mathrm{ppm} \mathrm{N}_{2}$ in the upper atmospheres of young Jupiters may augment the production of refractory condensable hydrocarbons through Titan-like ion chemistry. This possibility deserves further investigation, both experimentally and theoretically.

The dominant product of the coupled carbon-nitrogen photochemistry is $\mathrm{HCN}$, which forms through hypothesized schemes such as the following:

$$
\begin{aligned}
& \mathrm{N}_{2}+h \nu \rightarrow 2 \mathrm{~N} \\
& \mathrm{H}_{2} \mathrm{O}+h \nu \rightarrow \mathrm{OH}+\mathrm{H} \\
& \mathrm{OH}+\mathrm{H}_{2} \rightarrow \mathrm{H}_{2} \mathrm{O}+\mathrm{H} \\
& 2\left(\mathrm{H}+\mathrm{CH}_{4} \rightarrow \mathrm{CH}_{3}+\mathrm{H}_{2}\right) \\
& 2\left(\mathrm{~N}+\mathrm{CH}_{3} \rightarrow \mathrm{H}_{2} \mathrm{CN}+\mathrm{H}\right) \\
& 2\left(\mathrm{H}_{2} \mathrm{CN}+\mathrm{H} \rightarrow \mathrm{HCN}+\mathrm{H}_{2}\right) \\
& \hline \text { Net: } \mathrm{N}_{2}+2 \mathrm{CH}_{4} \rightarrow 2 \mathrm{HCN}+3 \mathrm{H}_{2} .
\end{aligned}
$$

Note that $\mathrm{N}_{2}$, not $\mathrm{NH}_{3}$, is the source of the nitrogen in this scheme, which is effective at high altitudes. That is why the HCN abundance can exceed the $\mathrm{NH}_{3}$ abundance at high altitudes in the $10 \mathrm{au}$ model shown in Figure 7. However, $\mathrm{NH}_{3}$ can also contribute to $\mathrm{HCN}$ formation through schemes such as the following that are more effective at lower stratospheric altitudes:

$$
\begin{aligned}
& \mathrm{NH}_{3}+h \nu \rightarrow \mathrm{NH}_{2}+\mathrm{H} \\
& 2\left(\mathrm{H}_{2} \mathrm{O}+h \nu \rightarrow \mathrm{OH}+\mathrm{H}\right) \\
& 2\left(\mathrm{OH}+\mathrm{H}_{2} \rightarrow \mathrm{H}_{2} \mathrm{O}+\mathrm{H}\right) \\
& \mathrm{H}+\mathrm{CH}_{4} \rightarrow \mathrm{CH}_{3}+\mathrm{H}_{2} \\
& \left.\mathrm{CH}_{3}+\mathrm{NH}_{2}+\mathrm{M} \rightarrow \mathrm{CH}_{3} \mathrm{NH}_{2}+\mathrm{M}\right) \\
& \mathrm{CH}_{3} \mathrm{NH}_{2}+\mathrm{H} \rightarrow \mathrm{CH}_{2} \mathrm{NH}_{2}+\mathrm{H}_{2} \\
& \mathrm{CH}_{2} \mathrm{NH}_{2}+\mathrm{H} \rightarrow \mathrm{CH}_{2} \mathrm{NH}+\mathrm{H}_{2} \\
& \mathrm{CH}_{2} \mathrm{NH}+\mathrm{H} \rightarrow \mathrm{H}_{2} \mathrm{CN}+\mathrm{H}_{2} \\
& \mathrm{H}_{2} \mathrm{CN}+\mathrm{H} \rightarrow \mathrm{HCN}+\mathrm{H}_{2} \\
& \text { Net: } \mathrm{NH}_{3}+\mathrm{CH}_{4} \rightarrow \mathrm{HCN}+3 \mathrm{H}_{2} \text {. }
\end{aligned}
$$

As shown in Figure 7, the coupled nitrogen-carbon photochemistry is more efficient with a greater UV flux from the host star.

Molecular nitrogen is fairly stable on young Jupiters. Photodissociation is only effective at wavelengths shorter than $\sim 1000 \AA$, so $\mathrm{N}_{2}$ can be shielded to some extent by the more abundant $\mathrm{H}_{2}, \mathrm{CO}$, and $\mathrm{H}_{2} \mathrm{O}$. In addition, the atomic $\mathrm{N}$ produced from $\mathrm{N}_{2}$ photolysis can go back to recycle the $\mathrm{N}_{2}$, through reactions such as $\mathrm{N}+\mathrm{OH} \rightarrow \mathrm{NO}+\mathrm{H}$, followed by $\mathrm{N}+\mathrm{NO} \rightarrow$ $\mathrm{N}_{2}+\mathrm{O}$. The production rate of $\mathrm{NO}$ through this process exceeds the loss rate, and NO appears as a minor high-altitude photochemical product on young Jupiters (Figure 7), especially for higher UV irradiation levels.

Ammonia, on the other hand, is much less stable than $\mathrm{N}_{2}$ because of weaker bonds, photolysis out to longer wavelengths $(\lambda \lesssim 2300 \AA)$, efficient reaction with atomic $\mathrm{H}$, and relatively inefficient recycling. The $\mathrm{NH}_{3}$ photolysis products can end up in $\mathrm{N}_{2}$ through reactions such as $\mathrm{N}+\mathrm{NH}_{2} \rightarrow \mathrm{NNH}+\mathrm{H}$, followed by $\mathrm{NNH} \rightarrow \mathrm{N}_{2}+\mathrm{H}$, or by $\mathrm{NH}_{2}+\mathrm{H} \rightarrow \mathrm{NH}+\mathrm{H}_{2}$, followed by $\mathrm{NH}+\mathrm{H} \rightarrow \mathrm{N}+\mathrm{H}_{2}$, and $\mathrm{N}+\mathrm{NO} \rightarrow \mathrm{N}_{2}+\mathrm{O}$. The nitrogen in the ammonia can also end up in $\mathrm{HCN}$, through reaction pathways such as scheme (6) above. As is apparent from Figure 7 , the $\mathrm{NH}_{3}$ in the upper stratosphere of young Jupiters becomes more depleted the higher the incident UV flux.

One other nitrogen-bearing photochemical product worth mentioning is $\mathrm{HC}_{3} \mathrm{~N}$, which is produced in the model through reaction of atomic $\mathrm{N}$ with $\mathrm{C}_{3} \mathrm{H}_{2}$ and $\mathrm{C}_{3} \mathrm{H}_{3}$ (e.g., Millar et al. 1991)—speculative reactions that may not be as efficient if we had more information about additional loss processes for these $\mathrm{C}_{3} \mathrm{H}_{x}$ species-and by $\mathrm{CN}+\mathrm{C}_{2} \mathrm{H}_{2} \rightarrow \mathrm{HC}_{3} \mathrm{~N}+\mathrm{H}$ (with the $\mathrm{CN}$ from $\mathrm{HCN}$ photolysis), which at least has a more convincing pedigree (e.g., Sims et al. 1993). Again, more $\mathrm{HC}_{3} \mathrm{~N}$ (and $\mathrm{CH}_{3} \mathrm{CN}$ ) are produced with higher incident UV fluxes. We have not included in the model reactions from the coupled photochemistry of $\mathrm{C}_{2} \mathrm{H}_{2}$ and $\mathrm{NH}_{3}$, which can produce a host of complex organic molecules (e.g., Keane et al. 1996; Moses et al. 2010), due to a lack of published thermodynamic properties for these molecules. However, heavier species such as acetaldazine, acetaldehyde hydrazone, and ethylamine may also form on young Jupiters due to this coupled chemistry, particularly on cooler, more highly UV irradiated planets. Unlike on our own solar-system gas giants, hydrazine $\left(\mathrm{N}_{2} \mathrm{H}_{4}\right)$ is not a major product of the ammonia photochemistry in our young-Jupiter models because the $\mathrm{NH}_{2}$ from ammonia photolysis preferentially reacts with the copious amounts of atomic $\mathrm{H}$ to produce $\mathrm{NH}$, and eventually $\mathrm{N}$ and $\mathrm{N}_{2}$, or with 
$\mathrm{CH}_{3}$ to form $\mathrm{CH}_{3} \mathrm{NH}_{2}$ and eventually $\mathrm{HCN}$. On Jupiter and Saturn, the coupled ammonia-methane photochemistry is less efficient due to the lack of $\mathrm{CH}_{3}$ present in the tropospheric region where $\mathrm{NH}_{3}$ is photolyzed (e.g., Kaye \& Strobel 1983; Moses et al. 2010). However, the hydrazine abundance is very sensitive to temperature and increases significantly as $T_{\text {eff }}$ decreases.

\subsubsection{Sensitivity of Disequilibrium Chemistry to Temperatures}

Finally, many photochemical products on directly imaged planets tend to be very sensitive to temperature-both the effective temperature of the planet, $T_{\text {eff }}$ (which on young Jupiters is controlled by the internal heat flux rather than radiation from the host star), and the temperature in the planet's stratosphere (i.e., the radiative region above the convecting troposphere). Note that because irradiation from the host star has less of an effect than internal heat flow on the upperatmospheric temperatures of these distant, young, hot, directly imaged planets, our generic young-Jupiter models with larger $T_{\text {eff }}$ have larger stratospheric temperatures, too (see Figure 1). As discussed previously, $T_{\text {eff }}$ affects the quenched abundances of the photochemically active parent molecules, which can in turn influence the production rate of disequilibrium photochemical "daughter" products. More importantly, the stratospheric temperatures affect the subsequent reaction rates of the photochemically produced molecules and radicals, as well as affect the height to which the photochemically active parent molecules are carried before molecular diffusion takes over and severely limits their abundance. The altitude variation of this homopause level can change the pressure at which photolysis occurs, thereby affecting subsequent pressure-dependent reactions. Figure 8 shows how the vertical profiles of some of the major photochemically active molecules in our models vary with temperature, for planets with $T_{\text {eff }}=600,900$, or $1200 \mathrm{~K}$, and $\log (g)=3.5(\mathrm{cgs}), K_{\text {deep }}=10^{6} \mathrm{~cm}^{2} \mathrm{~s}^{-1}$, orbiting at $68 \mathrm{au}$ from a star with the properties of HR 8799. Although variations in $T_{\text {eff }}$ have a relatively straightforward influence on the quenched species' abundances, the response to upper atmospheric temperatures is more complicated.

Smaller $T_{\text {eff }}$ results in larger quenched abundances of $\mathrm{CH}_{4}$, $\mathrm{NH}_{3}$, and $\mathrm{H}_{2} \mathrm{O}$ (all other factors being equal), and allows these molecules to be carried to higher homopause altitudes, so one might naively assume that these factors lead to greater abundances of photochemical products on cooler planets. However, photolysis in these young-Jupiter models is photonlimited rather than species-limited, and the column-integrated photolysis rate of water-which produces $\mathrm{H}$, as well as $\mathrm{OH}$, and thus drives much of the subsequent photochemistry for carbon, nitrogen, and oxygen species-is only slightly different for all three different $T_{\text {eff }}$ models shown in Figure 8. Instead, the critical factor is the efficiency of recycling of the parent species versus competing reactions to form other products. When temperatures are larger, recycling of water is more prevalent through reactions such as $\mathrm{OH}+\mathrm{H}_{2} \rightarrow \mathrm{H}_{2} \mathrm{O}+\mathrm{H}$, which has a high energy barrier and operates more effectively at high temperatures. Therefore, fewer reactive $\mathrm{OH}$ and $\mathrm{O}$ radicals are available to form oxygen-rich photochemical products such as $\mathrm{CO}_{2}, \mathrm{H}_{2} \mathrm{CO}, \mathrm{CH}_{3} \mathrm{OH}$, or $\mathrm{O}_{2}$ when temperatures are higher (see also Zahnle et al. 2016). Moreover, the $\mathrm{H}$ atom abundance increases as the upper-atmospheric temperature increases (due to the more efficient catalytic destruction of $\mathrm{H}_{2}$ following water photolysis), and the increased $\mathrm{H}$ atom abundance decreases the
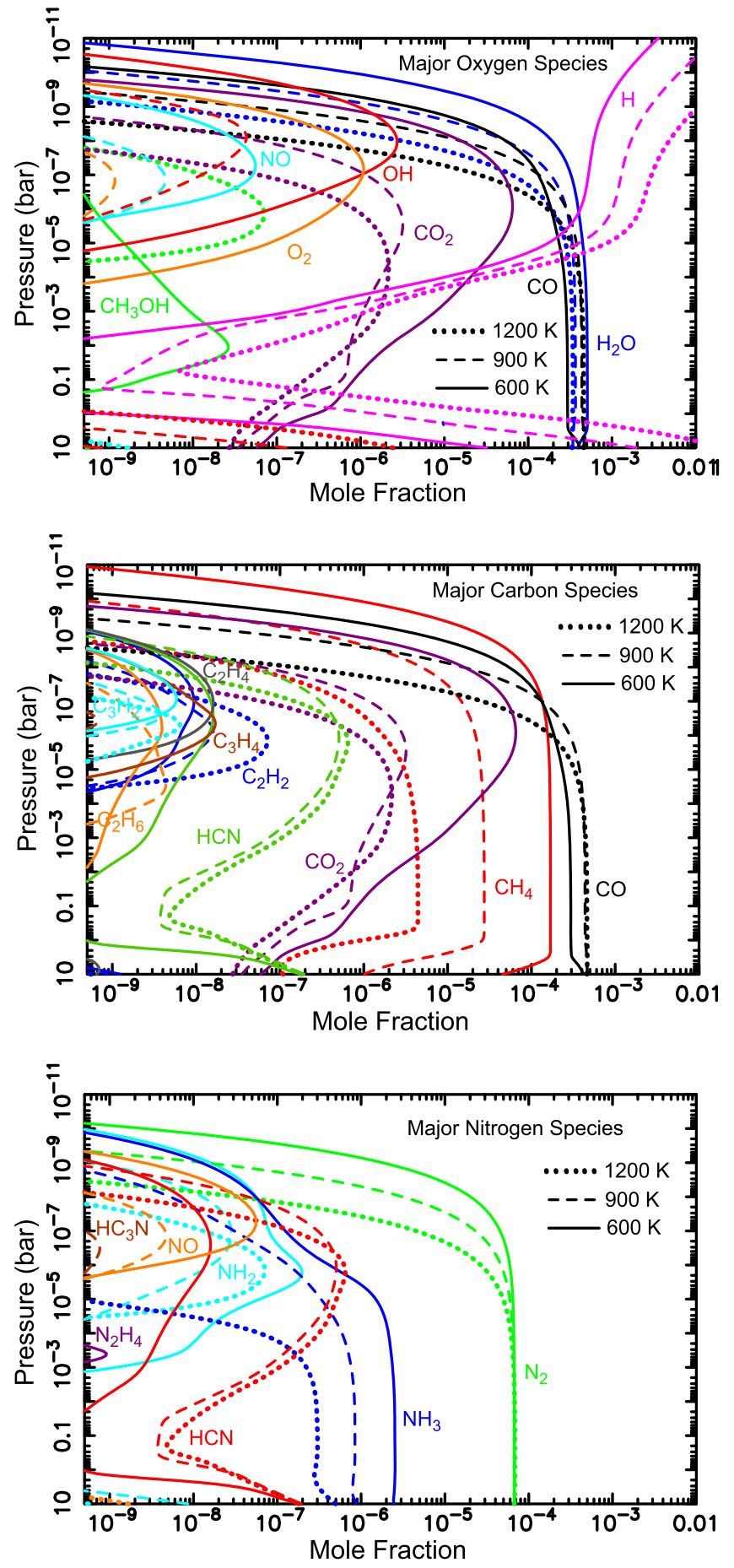

Figure 8. Vertical mixing-ratio profiles of several atmospheric species as a function of $T_{\text {eff }}$ for a planet with $g=10^{3.5} \mathrm{~cm} \mathrm{~s}^{-2}$ and $K_{\text {deep }}=10^{6} \mathrm{~cm}^{2} \mathrm{~s}^{-1}$ that is being irradiated by an HR 8799-like star at a distance of 68 au (dashed lines), for $T_{\text {eff }}=1200 \mathrm{~K}$ (dotted lines), $900 \mathrm{~K}$ (dashed lines), and $600 \mathrm{~K}$ (solid lines). Most disequilibrium photochemical products are synthesized more effectively in low- $T_{\text {eff }}$ atmospheres, but some photochemical products (most notably $\mathrm{HCN}$ and $\mathrm{C}_{2} \mathrm{H}_{2}$ ) become more abundant at higher $T_{\text {eff }}$.

stability of some photochemical products such as $\mathrm{CO}_{2}$ and $\mathrm{C}_{2} \mathrm{H}_{6}$.

On the other hand, the more efficient atomic $\mathrm{H}$ production at high temperatures leads to an overall increase in the production rate of reactive $\mathrm{CH}_{3}$ and $\mathrm{NH}_{2}$ radicals as the temperature increases, as a result of reactions like $\mathrm{H}+\mathrm{CH}_{4} \rightarrow \mathrm{CH}_{3}+\mathrm{H}_{2}$ 
and $\mathrm{H}+\mathrm{NH}_{3} \rightarrow \mathrm{NH}_{2}+\mathrm{H}_{2}$, and even though the reverse recycling reactions are also more effective at high temperatures, the nitrogen- and carbon-bearing products can still form at any temperature. The result is that some photochemical products, like $\mathrm{HCN}$ and $\mathrm{C}_{2} \mathrm{H}_{2}$ that have strong bonds and are more stable at high temperatures, are produced more efficiently at higher $T_{\text {eff }}$, while other species like $\mathrm{C}_{2} \mathrm{H}_{6}, \mathrm{C}_{3} \mathrm{H}_{4}$, and $\mathrm{N}_{2} \mathrm{H}_{4}$ are produced more efficiently at lower $T_{\text {eff }}$. The peak production altitude and overall shape of the mixing-ratio profiles can vary with $T_{\text {eff }}$, as well (see Figure 8).

As emphasized by Zahnle et al. (2016), the oxygen-bearing photochemical products are particularly sensitive to the upperatmospheric temperature, and the abundance of the oxygen species increases significantly when stratospheric temperatures fall below $\sim 250 \mathrm{~K}$. The rate coefficient for the water recycling reaction $\mathrm{OH}+\mathrm{H}_{2} \rightarrow \mathrm{H}_{2} \mathrm{O}+\mathrm{H}$ drops by almost three orders of magnitude with a reduction in temperature from $500 \mathrm{~K}$ to 200 $\mathrm{K}$ (Baulch et al. 2005). The reduced efficiency of $\mathrm{OH}+\mathrm{H}_{2} \rightarrow$ $\mathrm{H}_{2} \mathrm{O}+\mathrm{H}$ at low temperatures opens the door for efficient carbon oxidation, and $\mathrm{CO}+\mathrm{OH} \rightarrow \mathrm{CO}_{2}+\mathrm{H}$ becomes a competitive loss process for the $\mathrm{OH}$. As a result, neither $\mathrm{H}_{2} \mathrm{O}$ nor $\mathrm{CO}$ are as efficiently recycled in the colder atmospheres, and the $\mathrm{OH}+\mathrm{CO}$ reaction will proceed effectively until it depletes enough $\mathrm{CO}$ that the $\mathrm{OH}+\mathrm{H}_{2}$ reaction can again compete as a loss process for the $\mathrm{OH}$. One then sees a depletion of $\mathrm{H}_{2} \mathrm{O}$ and $\mathrm{CO}$ at high altitudes in the coldest models, with a concomitant increase in $\mathrm{CO}_{2}$ and other oxygen products like $\mathrm{O}_{2}$ and $\mathrm{CH}_{3} \mathrm{OH}$ that can form when $\mathrm{OH}$ does not effectively recycle back to water. Carbon dioxide becomes a spectroscopically significant photochemical product on colder young Jupiters (see Section 3.2), and the effect is further magnified the greater the incident UV flux.

Figure 9 provides further details showing how the photochemical products $\mathrm{CO}_{2}$ (top left), $\mathrm{HCN}$ (top right), $\mathrm{C}_{2} \mathrm{H}_{6}$ (bottom left), and $\mathrm{C}_{2} \mathrm{H}_{2}$ (bottom right) vary with changes in both $T_{\text {eff }}$ and $K_{\text {deep }}$, for planets with $\log (g)=3.5 \mathrm{cgs}$ located 68 au from a star like HR 8799. For the shape of the vertical $K_{z z}$ profiles we have assumed (see Figure 2), smaller $K_{\text {deep }}$ values also correspond to weaker eddy mixing in the lower stratosphere, which increases the residence time for photochemical products synthesized at higher altitudes, allowing them to build up to larger abundances. Therefore, most photochemical products exhibit increased abundances for smaller $K_{\text {deep }}$ values. One exception is $\mathrm{HCN}$, which has a more complicated dependence because larger $K_{\text {deep }}$ values favor larger quenched abundances of HCN; i.e., quenching, not just photochemistry, contributes to the overall abundance of $\mathrm{HCN}$. For any particular $K_{\text {deep }}$ value, the temperature dependence can be complicated, with $\mathrm{CO}_{2}$ exhibiting a major increase at the lowest temperatures for the reasons discussed above, $\mathrm{C}_{2} \mathrm{H}_{6}$ being favored at moderately low temperatures, and $\mathrm{C}_{2} \mathrm{H}_{2}$ and $\mathrm{HCN}$ being favored at $T_{\text {eff }} \approx 1200 \mathrm{~K}$.

In general, hydrocarbons such as $\mathrm{C}_{2} \mathrm{H}_{6}$ and $\mathrm{C}_{2} \mathrm{H}_{2}$ are not expected to become abundant enough to be observable on young Jupiters, except potentially for closer-in planets (i.e., those receiving a large UV flux) in combination with a more stagnant (lower $K_{z z}$ ) lower stratosphere and an increasingly well-mixed and colder $(\lesssim 250 \mathrm{~K})$ upper stratosphere, in which water recycling is less effective and the resulting $\mathrm{H}$ production is reduced. Low upper-atmospheric temperatures favor $\mathrm{C}_{2} \mathrm{H}_{6}$ over $\mathrm{C}_{2} \mathrm{H}_{2}$, while higher temperatures favor $\mathrm{C}_{2} \mathrm{H}_{2}$. The quenched $\mathrm{HCN}$ abundance reaches potentially observable abundances of a few $\times 10^{17} \mathrm{~cm}^{-2}$ above $100 \mathrm{mbar}$ for large $K_{\text {deep }}\left(\gtrsim 10^{9} \mathrm{~cm}^{2} \mathrm{~s}^{-1}\right)$, and a high UV flux combined with moderate $T_{\text {eff }}$ of 1100-1300 K would provide an increased photochemical component on top of that that quenched HCN. Carbon dioxide is the big winner from a disequilibriumchemistry standpoint, with observable quantities (see Section 3.2) of greater than $10^{18} \mathrm{~cm}^{-2}$ above 100 mbar being produced through both quenching and photochemistry in all the models studied, with a column abundance greater than $10^{19}$ $\mathrm{cm}^{-2}$ above 100 mbar forming in the planets with cooler, more stagnant lower stratospheres.

\subsection{Generic Directly Imaged Planets: Spectra}

We show selected spectra from our directly imaged planets in Figures $10 \& 11$. These synthetic spectra were generated from the forward radiative-transfer model described in Line et al. (2013, 2014, 2015). First, Figure 10 shows results from two generic models with different quenched abundances of $\mathrm{CH}_{4}$ and $\mathrm{CO}$. Both planets are assumed to be $39 \mathrm{pc}$ from Earth, with surface gravities of $10^{4} \mathrm{~cm} \mathrm{~s}^{-2}$, a radius of $1.2 R_{J}$, and a uniform gray absorbing aerosol layer with a base located where the thermal profile crosses the $\mathrm{MgSiO}_{3}$ condensation curve and a total optical depth of unity between 1 bar and $10^{-4}$ bars. Both planets are assumed to orbit 68 au from a star with properties of HR 8799. The planet shown in the left panel has $T_{\text {eff }}=600 \mathrm{~K}$ and $K_{\text {deep }}=10^{5} \mathrm{~cm}^{2} \mathrm{~s}^{-1}$, for which the quenched $\mathrm{CH}_{4}$ mixing ratio is 2.4 times that of $\mathrm{CO}$ (see Figure 5). The planet in the right panel has $T_{\text {eff }}=1000 \mathrm{~K}$ and $K_{\text {deep }}=10^{7} \mathrm{~cm}^{2} \mathrm{~s}^{-1}$, such that the quenched $\mathrm{CO}$ mixing ratio is 18 times that of $\mathrm{CH}_{4}$. Absorption features of $\mathrm{H}_{2} \mathrm{O}$ are readily apparent in the spectra of both planets in bands near $\sim 1.4, \sim 1.8-1.9, \sim 2.6-2.8$, and the $\sim 5.5-7.5 \mu \mathrm{m}$ region, and $\mathrm{CO}$ absorption features are apparent in both plots in the $\sim 4.5-4.8 \mu \mathrm{m}$ region. Although $\mathrm{CH}_{4}$ absorption features are also obvious in both plots, the bands at $2.3,3.3$, and $7.7 \mu \mathrm{m}$ are deeper for the cooler planet, with its larger quenched methane abundance. The cooler planet also has a larger column of photochemically produced $\mathrm{CO}_{2}$, which shows up most distinctly in the $4.2-4.3 \mu \mathrm{m}$ absorption bands on both planets, as well as more subtlely in the $2.7-2.8 \mu \mathrm{m}$ region on the warmer planet and the $\sim 14-16 \mu$ m region on the cooler planet. Absorption in the 4.2-4.3 $\mu \mathrm{m} \mathrm{CO} \mathrm{CO}_{2}$ bands should be particularly apparent on young Jupiters, trending toward greater absorption for lower $T_{\text {eff }}$. HCN is abundant enough on the warmer, more rapidly mixed planet (see Figure 9) to have a minor effect on the spectrum at $3 \mu \mathrm{m}$, while other photochemical products such as $\mathrm{C}_{2} \mathrm{H}_{2}$ are not abundant enough to notably affect the spectra for either of these generic young Jupiters considered.

Figure 11 further illustrates how the spectra of our generic young Jupiters changes as a function of $T_{\text {eff }}$. In this figure, we plot the synthetic spectra from the photochemical models shown in Figure 8-these planets are assumed to orbit 68 au from a star with properties similar to HR 8799, and have $g=3200 \mathrm{~cm} \mathrm{~s}^{-2}, K_{\text {deep }}=10^{6} \mathrm{~cm}^{2} \mathrm{~s}^{-1}$, and $T_{\text {eff }}=600,900$, or $1200 \mathrm{~K}$. For the spectral calculations, we again assume that the systems are located 39 pc from Earth, with planetary radii $=1.0 R_{J}$ and uniform gray absorbing clouds with optical depths of one between the base of the $\mathrm{MgSiO}_{3}$ condensation region and the top of the atmosphere. The cooler planet contains more quenched $\mathrm{H}_{2} \mathrm{O}$ and $\mathrm{CH}_{4}$ and possesses a colder stratosphere, so the absorption bands due to these species are therefore deeper. The cooler planet also has more quenched 

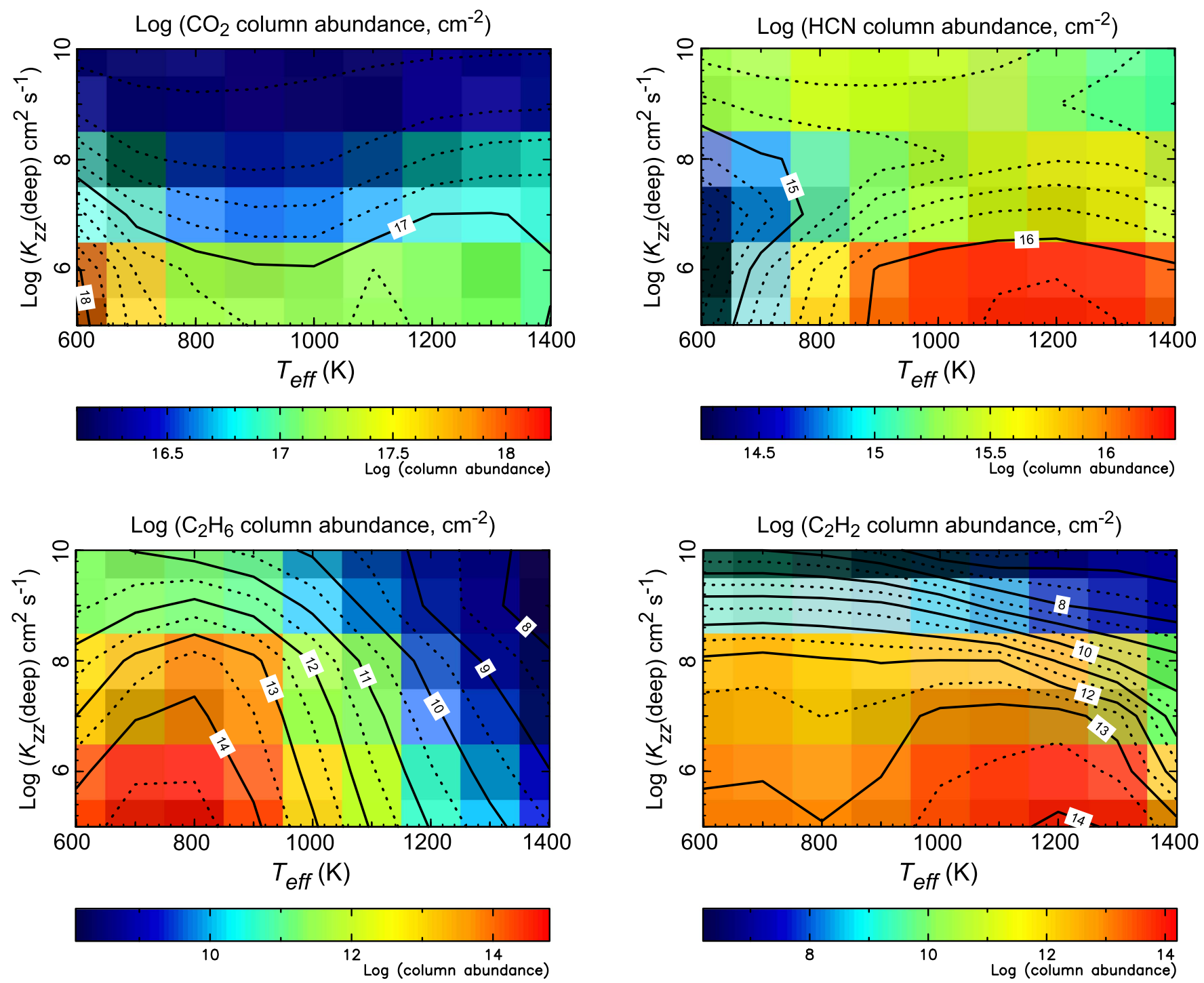

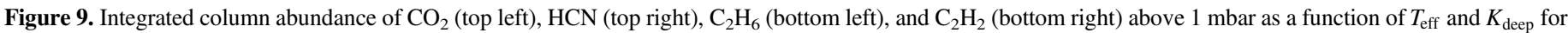

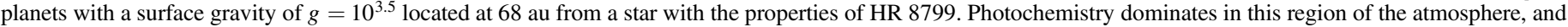
different species exhibit a complicated sensitivity to both $T_{\text {eff }}$ and $K_{\text {deep }}$.

$\mathrm{NH}_{3}$, which shows up readily near $10.4 \mu \mathrm{m}$, and more photochemically produced $\mathrm{CO}_{2}$, which is notable in the $4.2-4.3$ and $\sim 15 \mu \mathrm{m}$ bands.

The influence of photochemically produced $\mathrm{CO}_{2}$ on the emission spectrum of young Jupiters diminishes strongly with increasing $T_{\text {eff }}$ and increasing $K_{z z}$ in the stratosphere, and is, in particular, highly sensitive to the stratospheric temperature, as discussed in Section 3.1.4. For sufficiently large stratospheric $K_{z z}$ and temperatures, an increased UV irradiation level does not overcome the tendency toward small overall $\mathrm{CO}_{2}$ column abundances. For example, Figure 12 shows how spectra from the $T_{\text {eff }}=1000 \mathrm{~K}, \log (g)=4.0, K_{\text {deep }}=10^{7} \mathrm{~cm}^{2} \mathrm{~s}^{-1}$ models from Figure 7 vary with orbital distance ranging from 10, 32, and $100 \mathrm{au}$. The spectra are similar for all three planets. There is a slight difference in the $4.2-4.3 \mu \mathrm{m}$ region due to increased $\mathrm{CO}_{2}$ absorption for the shorter-period planets, but these differences are small. In general, the spectra of young directly imaged giant planets will be dominated by quenched $\mathrm{H}_{2} \mathrm{O}$, $\mathrm{CH}_{4}$, and $\mathrm{CO}$, but absoption features due to photochemically produced species such as $\mathrm{CO}_{2}$ can be important when $T_{\text {eff }}$ is small, lower-stratospheric eddy mixing coefficients are small (which allow larger column abundances of photochemical species to build up), and UV irradiation levels are large.

\section{3. $H R 8799 b$}

Of the four planets detected in the HR 8799 system (Marois et al. 2008, 2010), HR 8799 b is the farthest away from the host star (68 au, Maire et al. 2015) and seems to be the smallest and coolest (e.g., Marois et al. 2008). Most comparisons of spectral models with observational data favor $T_{\text {eff }}$ in the broad range $700-1200 \mathrm{~K}$ and $\log (g)=3.0-4.5 \mathrm{cgs}$ for HR $8799 \mathrm{~b}$ (Marois et al. 2008; Hinz et al. 2010; Barman et al. 2011a, 2015; Currie et al. 2011, 2014; Galicher et al. 2011; Madhusudhan et al. 2011; Marley et al. 2012; Skemer et al. 2012; Ingraham et al. 2014; Rajan et al. 2015). The broad range here stems from degeneracies between $T_{\text {eff }}, \log (g)$, assumed cloud properties, planetary radius, and metallicity. Moreover, the models tend to have difficulty simultaneously fitting the short-wavelength infrared spectra $(1-2.5 \mu \mathrm{m})$, which show evidence for deeper molecular absorptions, and the longer-wavelength mid-infrared 

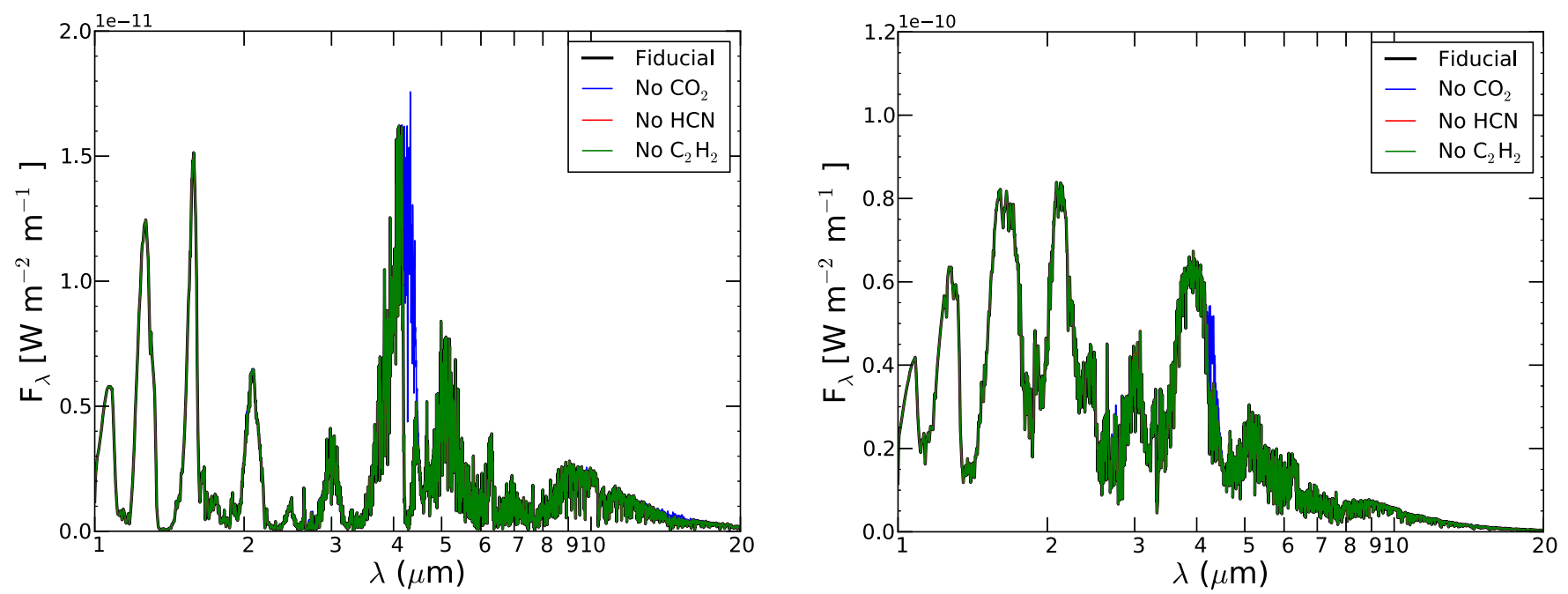

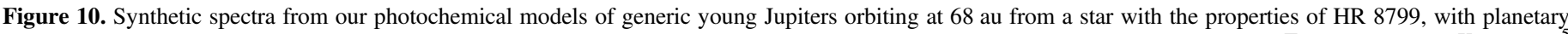

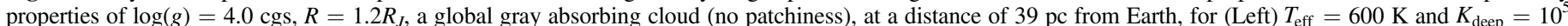

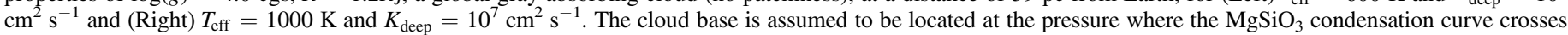

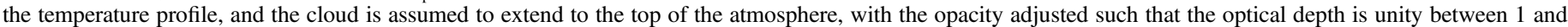

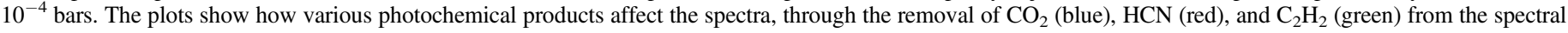
calculations. Of these photochemical products, only $\mathrm{CO}_{2}$ affects the spectra significantly at near-IR wavelengths.

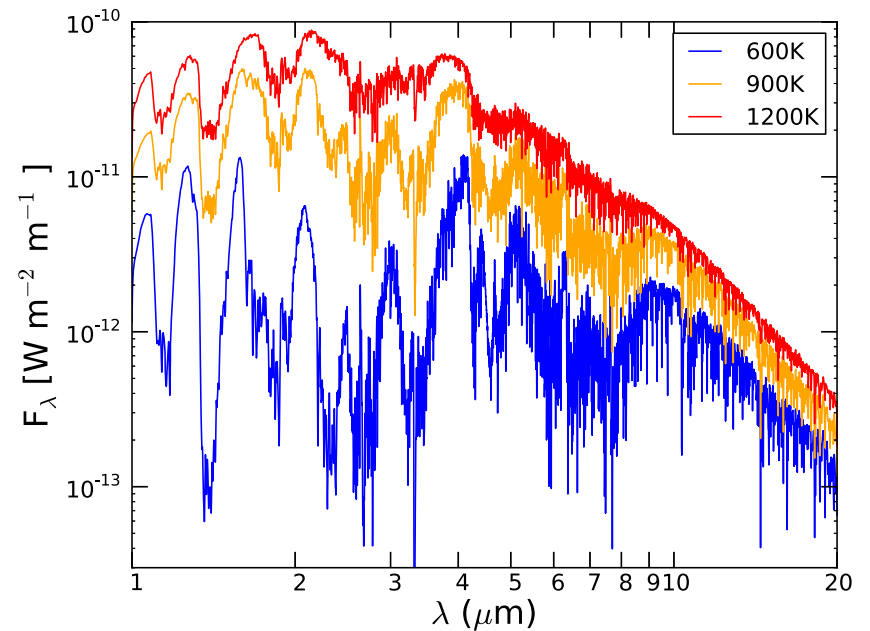

Figure 11. Synthetic spectra from our photochemical models of generic young Jupiters orbiting 68 au from a star with the properties of HR 8799, with surface gravities $g=3200 \mathrm{~cm} \mathrm{~s} \mathrm{~s}^{-2}$, eddy $K_{\text {deep }}=10^{6} \mathrm{~cm}^{2} \mathrm{~s}^{-1}$, and effective temperatures $T_{\text {eff }}=600 \mathrm{~K}$ (blue), $900 \mathrm{~K}$ (orange), and $1200 \mathrm{~K}$ (red). These models correspond to the ones shown in Figure 8. For the purpose of the spectral calculations, we have assumed that the planets have radii $=1.0 R_{J}$, are located $39 \mathrm{pc}$ from Earth, and possess uniform gray absorbing clouds with optical depths of one between the base of the $\mathrm{MgSiO}_{3}$ condensation region and the top of the atmosphere. Note that the absorption in most of the molecular bands (e.g., $\mathrm{H}_{2} \mathrm{O}, \mathrm{CH}_{4}, \mathrm{NH}_{3}$, and $\mathrm{CO}_{2}$ ) increases as $T_{\text {eff }}$ decreases (cf. Figure 8).

photometric $(3-5 \mu \mathrm{m})$, which exhibit flatter spectral behavior. These difficulties complicate the derivation of planetary properties. The best-fit models typically seem to require thick but patchy clouds, and the spectrum of HR $8799 \mathrm{~b}$ is distinctly different from brown dwarfs with the same effective temperature.

For our HR 8799 b models, we adopt the recent constraints of Barman et al. (2015) $\left(T_{\text {eff }}=1000 \pm 100 \mathrm{~K}\right.$ and $\log$ $(g)=3.5 \pm 0.5 \mathrm{cgs})$ because their analysis of the mediumresolution $H$ - and $K$-band data with the OSIRIS instrument at Keck have provided the best available constraints on the

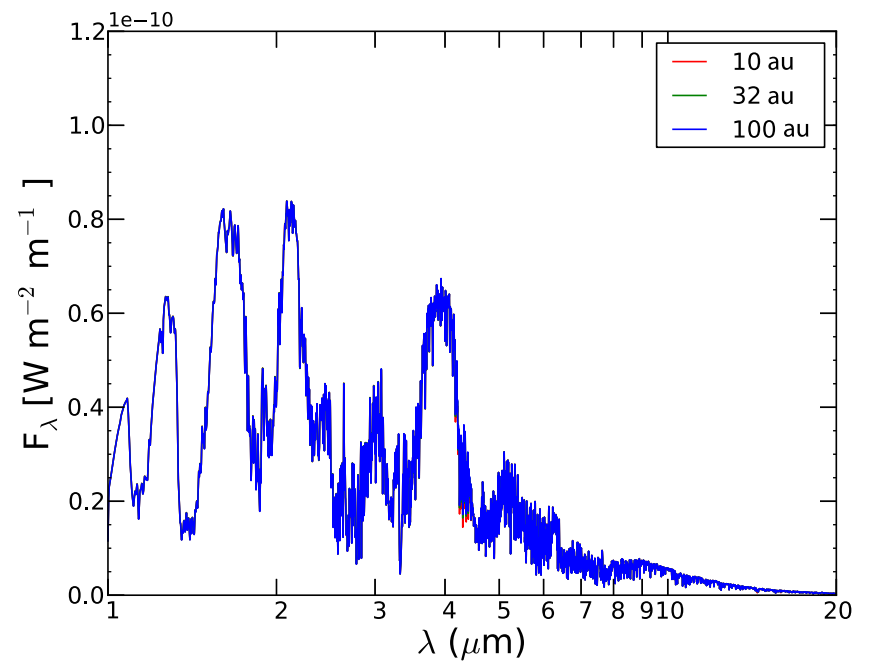

Figure 12. Synthetic spectra from our photochemical models of generic young Jupiters with $T_{\text {eff }}=1000 \mathrm{~K}, g=10^{4} \mathrm{~cm} \mathrm{~s}^{-2}, K_{\text {deep }}=10^{7} \mathrm{~cm}^{2} \mathrm{~s}^{-1}$, orbiting a star with the properties of HR 8799 at 10 au (red), 32 au (green), and 100 au (blue). These models correspond to the ones shown in Figure 7. For the purpose of the spectral calculations, we have assumed that the planets have radii $=1.2 R_{J}$, are located $39 \mathrm{pc}$ from Earth, and possess uniform gray absorbing clouds with optical depths of one between 1 and $10^{-4}$ mbar. Note that at this relatively high $T_{\text {eff }}$ and $K_{\text {deep }}$ the photochemical products have little impact on the spectrum, except for the minor increase in $\mathrm{CO}_{2}$ absorption at $4.2-4.3 \mu \mathrm{m}$ in the shorter-period model, due to its greater photochemical production and correspondingly larger $\mathrm{CO}_{2}$ abundance.

abundances of $\mathrm{CH}_{4}, \mathrm{H}_{2} \mathrm{O}$, and $\mathrm{CO}$. For consistency with the Barman et al. (2015) modeling procedure and their preferred restriction of $\mathrm{C}$ and $\mathrm{O}$ abundances to possible sequences derived from the Öberg et al. (2011) disk chemical evolution model, we also adopt a slightly super-solar $\mathrm{C} / \mathrm{O}$ ratio of $0.65-0.7$ for these models, and metallicities of $\sim 0.6-1.0$ times solar.

Figure 13 shows the results from one of our HR $8799 \mathrm{~b}$ models. In this model, we have assumed $T_{\text {eff }}=1000 \mathrm{~K}$, $g=3000 \mathrm{~cm} \mathrm{~s}^{-2}$ (with assumed mass $1.9 M_{J}$ ), and a solar 
Eddy Diffusion Coefficient $\left(\mathrm{cm}^{2} \mathrm{~s}^{-1}\right)$
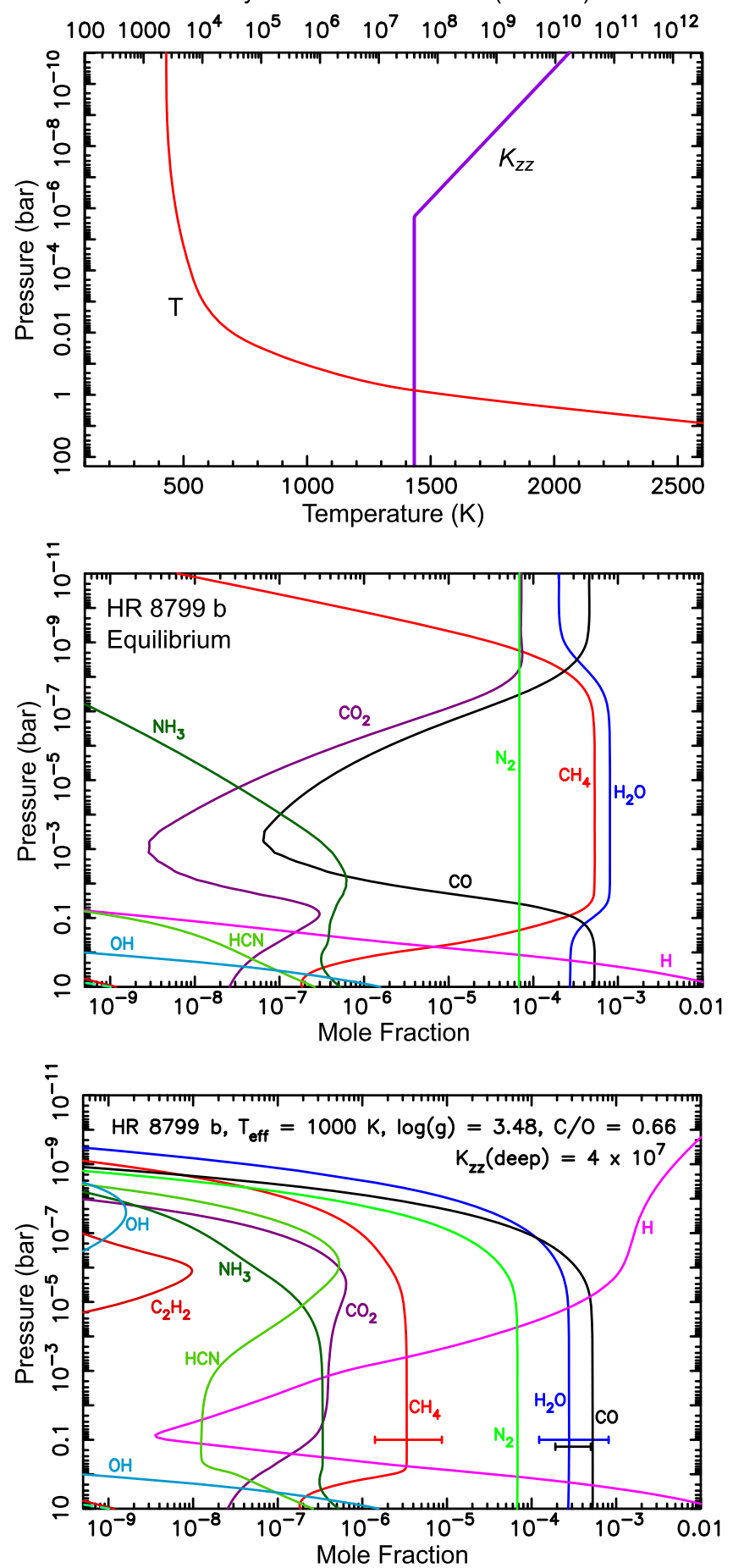

Figure 13. Chemical model for HR 8799 b assuming $T_{\text {eff }}=1000 \mathrm{~K}$, $g=3000 \mathrm{~cm} \mathrm{~s}^{-2}$ (assumed $M=1.9 M_{J}$ ), and solar metallicity, except $\mathrm{C} / \mathrm{O}=0.66$ : (Top) The temperature profile (red curve, bottom axis) from the radiative-convective equilibrium model of Marley et al. (2012) assuming the above bulk constraints, and the eddy diffusion coefficient profile (purple curve, top axis) adopted in the photochemical model; (Middle) the predicted thermochemical equilibrium mixing-ratio profiles for the major oxygen, carbon, and nitrogen species, as labeled, for the assumed pressure-temperature profile; (Bottom) mixing-ratio profiles predicted from our thermo/photochemical kinetics and transport model for the above thermal structure, $K_{z z}$ profile, and assumed bulk elemental composition. The line segments in the bottom plot are the observational constraints for $\mathrm{CH}_{4}$ (red), $\mathrm{H}_{2} \mathrm{O}$ (blue), and $\mathrm{CO}$ (black) from Barman et al. (2015). metallicity atmosphere except for a $\mathrm{C} / \mathrm{O}$ ratio of 0.66 , and we have used the radiative-convective equilibrium model of Marley et al. (2012) to define the temperature structure. With this thermal structure, the quenched $\mathrm{CH}_{4}$ abundance falls within the $1.4 \times 10^{-6}-8.7 \times 10^{-6}$ mixing-ratio constraints provided by Barman et al. (2015) when $\log \left(K_{\text {deep }}\right) \approx 6-9$, with a best fit for $K_{\text {deep }}=4 \times 10^{7} \mathrm{~cm}^{2} \mathrm{~s}^{-1}$. Figure 13 demonstrates that the $\mathrm{CO}$ mixing ratio is expected to be much larger than the $\mathrm{CH}_{4}$ mixing ratio on $\mathrm{HR} 8799 \mathrm{~b}$ as a result of transport-induced quenching. Similarly, the quenched $\mathrm{N}_{2}$ abundance is much greater than that of $\mathrm{NH}_{3}$, and $\mathrm{H}_{2} \mathrm{O}$ quenches at a mixing ratio a factor of $\sim 3$ smaller than equilibrium predictions. As expected (see Section 3.1), the $\mathrm{CO}_{2}$ and $\mathrm{HCN}$ abundances are also significantly enhanced in comparison to chemical equilibrium as a result of quenching of the dominant oxygen, carbon, and nitrogen species (see also Moses et al. 2011). The coupled carbon-oxygen and carbonnitrogen photochemistry described in Section 3.1) leads to an additional peak in the $\mathrm{CO}_{2}$ and $\mathrm{HCN}$ abundances at high altitudes, which for the case of $\mathrm{HCN}$ adds notably to the stratospheric column abundance. Hydrocarbons such as $\mathrm{C}_{2} \mathrm{H}_{2}$ and $\mathrm{C}_{2} \mathrm{H}_{6}$ and key radicals such as $\mathrm{OH}$ and $\mathrm{NH}_{2}$ are produced from high-altitude photochemistry, but these species are less stable in the lower stratosphere, and they never reach observable column abundances.

Overall, although disequilibrium quenching is very important in controlling the atmospheric composition of HR $8799 \mathrm{~b}$ -including controlling the abundance of minor species not typically considered in simple quenching models_-photochemistry itself is less important due to relatively warm stratospheric temperatures (which tend to decrease the stability of photochemical products) and the mild UV flux received by HR 8799 b. If the lower-stratospheric eddy $K_{z z}$ values were much lower than we have assumed here, then the column abundance of key photochemical products like $\mathrm{C}_{2} \mathrm{H}_{x}$ hydrocarbons could be increased, although it is still unlikely that they could achieve observable values.

Other HR 8799 b models developed with different assumptions about the thermal structure and other planetary parameters produce similar results. For colder (warmer) thermal structures, it takes a larger (smaller) $K_{\text {deep }}$ to quench $\mathrm{CH}_{4}$ at the same abundance as in the above model. As an example, Figure 14 shows the results for two photochemical models that assume $T_{\text {eff }}=1000 \mathrm{~K}, g=3162 \mathrm{~cm} \mathrm{~s}^{-2}$, a C/O ratio of 0.7 , a subsolar metallicity (i.e., $\sim 0.63$ times the solar $\mathrm{O} / \mathrm{H}$ of Grevesse et al. 2007), a thermal structure that is taken from Barman et al. (2015), and an assumed $K_{\text {deep }}$ that is $10^{8} \mathrm{~cm}^{2} \mathrm{~s}^{-1}$ (solid curves) or $10^{9} \mathrm{~cm}^{2} \mathrm{~s}^{-1}$ (dotted curves). These models are cooler everywhere than the one shown in Figure 13, and so it takes a larger $K_{\text {deep }}$ to quench $\mathrm{CH}_{4}$ at the same abundance as the previous model. If the eddy diffusion coefficient were to remain high in the stratosphere, as in the models shown here, then the photochemical species produced at high altitudes could diffuse rapidly through the stratosphere to deeper, warmer levels, where they would readily be converted back to the major quenched species. So again, photochemistry does not have much of an effect on the spectroscopically active molecules for these HR 8799 b models. However, transportinduced quenching does play a major role in shaping atmospheric composition, including for the species $\mathrm{H}_{2} \mathrm{O}, \mathrm{CO}$, $\mathrm{CH}_{4}, \mathrm{CO}_{2}, \mathrm{~N}_{2}, \mathrm{NH}_{3}$, and $\mathrm{HCN}$. Quenching on a lower-gravity planet readily explains why the observed $\mathrm{CH}_{4}$ absorption is so 

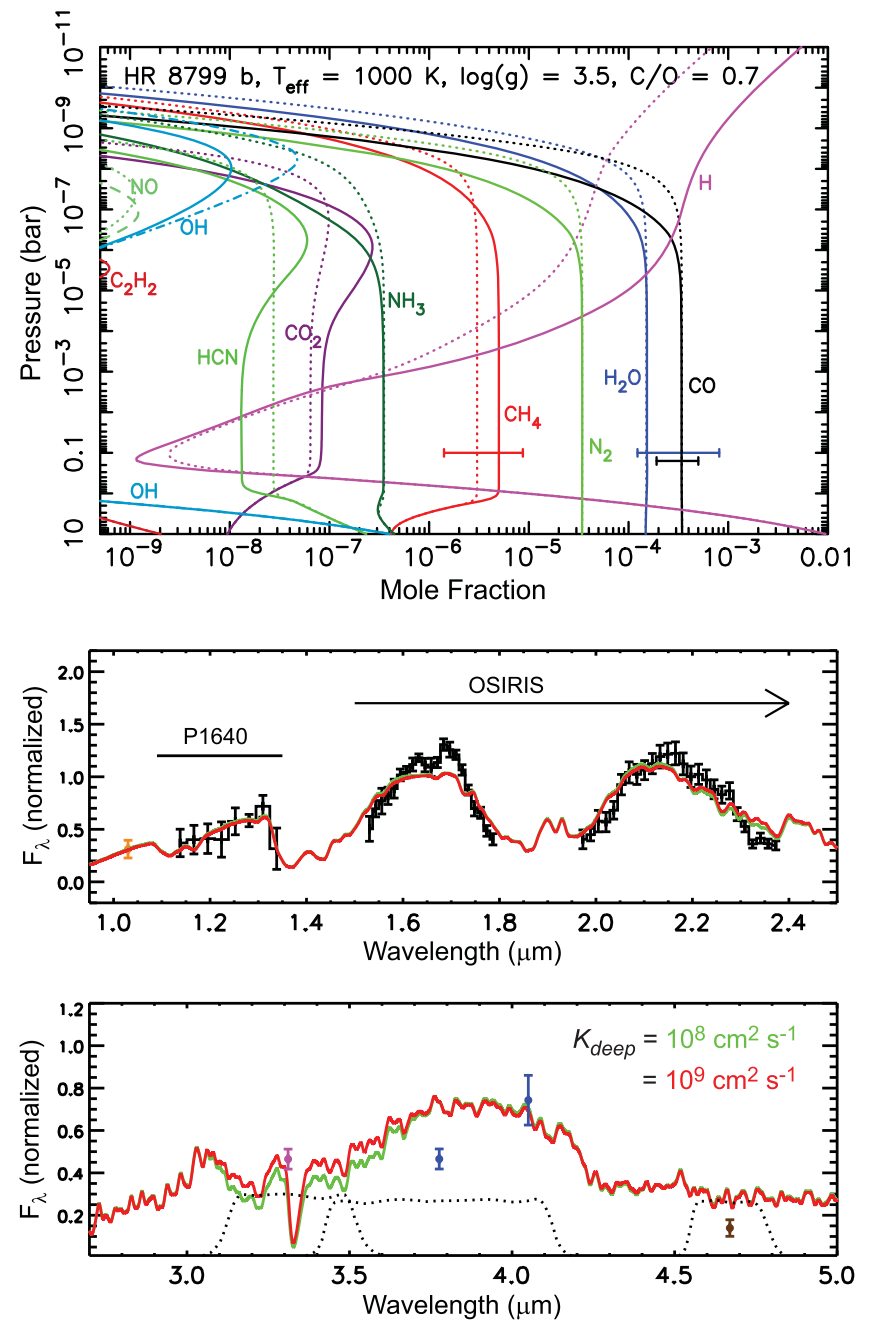

Figure 14. (Top) Model results for HR $8799 \mathrm{~b}$ assuming $T_{\mathrm{eff}}=1000 \mathrm{~K}, \log$ $(g)=3.5 \mathrm{cgs}, \mathrm{C} / \mathrm{O}=0.7$, a subsolar metallicity, a temperature profile from Barman et al. (2015), and $K_{\text {deep }}=10^{8} \mathrm{~cm}^{2} \mathrm{~s}^{-1}$ (solid curves) and $10^{9} \mathrm{~cm}^{2}$ $\mathrm{s}^{-1}$ (dotted curves). (Bottom two panels) HR $8799 \mathrm{~b}$ observations (black data points with error bars; see text) compared with synthetic spectra generated from our thermo/photochemical kinetics and transport models from the top panel, for $K_{\text {deep }}=10^{8} \mathrm{~cm}^{2} \mathrm{~s}^{-1}$ (green) and $10^{9} \mathrm{~cm}^{2} \mathrm{~s}^{-1}$ (red); see text and Barman et al. (2015) for details.

much less significant on HR $8799 \mathrm{~b}$ than on brown dwarfs of similar effective temperatures (see also Barman et al. 2011a, 2015; Zahnle \& Marley 2014). Although $K_{\text {deep }}$ can in theory be constrained by comparing disequilibrium models like these to observations, in practice the thermal structure of the planet is uncertain enough that firm constraints are not possible. We simply conclude that the deep-atmospheric mixing is strong $\left(K_{\text {deep }}>10^{7} \mathrm{~cm}^{2} \mathrm{~s}^{-1}\right)$ on HR $8799 \mathrm{~b}$, consistent with that expected from convection on a planet with a strong internal heat source (e.g., Stone 1976).

Figure 14 also shows how synthetic spectra from these models compare to actual observations. In order of shorter to longer wavelengths in this figure, we plot the $\mathrm{z} / \mathrm{Y}$-band flux of Currie et al. (2011) in orange, the low-resolution P1640 J-band spectrum of Oppenheimer et al. (2013) in black, the $H$-band OSIRIS spectrum of Barman et al. (2011a) in black, the $K$-band OSIRIS spectrum of Barman et al. (2015) in black, and the longer-wavelength photometric data of Skemer et al. (2012) in pink, Currie et al. (2014) in blue, and Galicher et al. (2011) in brown. A small scaling was applied to the $H$-band portion of the P1640 spectrum to get it to match the $H$-band OSIRIS spectrum, as described in Barman et al. (2015). For other modeling assumptions, see Barman et al. (2015). The quenched $\mathrm{CH}_{4}$ abundance is sensitive to the assumed deep eddy diffusion coefficient $K_{\text {deep }}$, and Figure 14 shows that in the near-infrared, spectral observations in the $3.1-3.5 \mu \mathrm{m}$ region are best suited to constraining the methane mixing ratio and hence $K_{\text {deep }}$. Although current ground-based and Hubble Space Telescope observations can provide sufficient spectral information to loosely constrain $\mathrm{CH}_{4}$ and thus $K_{\text {deep }}$ (e.g., Konopacky et al. 2013; Barman et al. 2015), model degeneracies will be more easily broken with the broader wavelength coverage and moderate spectral resolution provided by the James Webb Space Telescope (JWST) or other space-based instrumentation with coronographic spectroscopy capabilities.

The column abundances of key species in our full range of HR $8799 \mathrm{~b}$ models are listed in Table 1. Water is the dominant infrared opacity source and is readily detected in HR 8799 b spectra. Methane and carbon monoxide have also been detected (e.g., Barman et al. 2011a, 2015; Currie et al. 2011). Tentative detections of $\mathrm{NH}_{3}$ and/or $\mathrm{C}_{2} \mathrm{H}_{2}$, and $\mathrm{CO}_{2}$ or $\mathrm{HCN}$ have been reported by Oppenheimer et al. (2013) in 1.0-1.8 $\mu \mathrm{m}$ spectra of the planet. Many of these tentative detections are inconsistent with our HR 8799 b models. For example, $\mathrm{C}_{2} \mathrm{H}_{2}$ in our photochemical models never becomes abundant enough to be detectable on HR 8799 b for any of the infrared bands, including the relatively strong ones near 13.6 and $\sim 3 \mu \mathrm{m}$. Carbon dioxide in the model is not abundant enough to be detectable in the $1-1.8 \mu \mathrm{m}$ range, where the bands are weak, but it should be detectable in the stronger bands between 4-4.5 $\mu \mathrm{m}$ and near $15 \mu \mathrm{m} ; \mathrm{CO}_{2}$ may also be detectable in the $\sim 2.7-2.8 \mu \mathrm{m}$ range if the photosphere extends down to $\sim 1 \mathrm{bar}$, but that may be problematic given that clouds are inferred to be present. Hydrogen cyanide is potentially detectable in bands near 2.5, 3, and 6.8-7.4 $\mu \mathrm{m}$ if the photosphere extends deep, with a more likely stratospheric detection in the $14-\mu \mathrm{m}$ band; however, $\mathrm{HCN}$ is not predicted to be abundant enough to be detectable in the $1-1.8 \mu \mathrm{m}$ region observed by Oppenheimer et al. (2013). Similarly, if the photosphere extends below $\sim 1$ bar, $\mathrm{NH}_{3}$ may be detectable near $\sim 1.5 \mu \mathrm{m}, \sim 2 \mu \mathrm{m}, \sim 3 \mu \mathrm{m}$, and $\sim 6.15 \mu \mathrm{m}$, but has the best chance of being detected in the stratosphere in the stronger bands in the $9-11 \mu \mathrm{m}$ region. Methane should be detectable in the $\sim 1.6$ and $2.3 \mu \mathrm{m}$ bands if the obscuring clouds are confined to altitudes below $\sim 100$ mbar (and in fact $\mathrm{CH}_{4}$ has been detected in the $2.3 \mu \mathrm{m}$ band, Barman et al. 2011a, 2015), with an even better chance of being detected in the stronger $3.3 \mu \mathrm{m}$ band (see Currie et al. 2011, and Figure 14) and the $7.7 \mu \mathrm{m}$ band. The $\mathrm{CO}$ band in the 4.5-4.9 $\mu \mathrm{m}$ region should produce significant absorption in HR $8799 \mathrm{~b}$ spectra, and the band near $2.3-2.4 \mu \mathrm{m}$ should also be observable (see Barman et al. 2015) and may help constrain cloud heights/thicknesses; however, moderate-resolution spectra are required, as some of the lines in this band overlap with $\mathrm{H}_{2} \mathrm{O}$ and $\mathrm{CH}_{4}$ lines, complicating identification (Barman et al. 2015).

\subsection{Eri $b$}

51 Eridani b, a 20 Myr old exoplanet that is cooler and closer to its star than HR 8799 b, was recently discovered with the Gemini Planet Imager (GPI; Macintosh et al. 2015). As with several other cool young Jupiters, the near-infrared flux 
Table 1

Column Abundances for the HR 8799 b Models

\begin{tabular}{lcrr}
\hline \hline Species & $\begin{array}{c}\text { Column Abundance } \\
\text { Above 10 mbar } \\
\left(\mathrm{cm}^{-2}\right)\end{array}$ & $\begin{array}{c}\text { Column Abundance } \\
\text { Above 100 mbar } \\
\left(\mathrm{cm}^{-2}\right)\end{array}$ & $\begin{array}{c}\text { Column Abundance } \\
\text { Above } 1 \text { bar } \\
\left(\mathrm{cm}^{-2}\right)\end{array}$ \\
\hline $\mathrm{CH}_{4}$ & $(2-3) \times 10^{18}$ & $(2-3) \times 10^{19}$ & $(2-3) \times 10^{20}$ \\
$\mathrm{C}_{2} \mathrm{H}_{2}$ & $(0.01-5) \times 10^{12}$ & $(0.01-5) \times 10^{12}$ & $(0.8-8) \times 10^{14}$ \\
$\mathrm{H}_{2} \mathrm{O}$ & $(1-3) \times 10^{20}$ & $(1-3) \times 10^{21}$ & $(1-3) \times 10^{22}$ \\
$\mathrm{CO}$ & $(2.8-4.6) \times 10^{20}$ & $(2.8-4.5) \times 10^{21}$ & $(0.4-1) \times 10^{19}$ \\
$\mathrm{CO}_{2}$ & $(0.5-4) \times 10^{17}$ & $(2.5-3) \times 10^{18} \times 10^{18}$ & $(2.5-3) \times 10^{19}$ \\
$\mathrm{NH}_{3}$ & $(2.5-3) \times 10^{17}$ & $(1-2.5) \times 10^{17}$ & $(1-2.5) \times 10^{18}$ \\
$\mathrm{HCN}$ & $(1-3) \times 10^{16}$ & & \\
\hline
\end{tabular}

Note. Models assume $\log (g)=3.47-3.5$ cgs.

and emission spectrum of 51 Eri b is difficult to reproduce theoretically without invoking cloudy or partial-cloud-covered atmospheres (Macintosh et al. 2015). The spectra show evidence for strong methane and water absorption (Macintosh et al. 2015); however, $\mathrm{CH}_{4}$ is underabundant in comparison with chemical equilibrium, indicating that quenching is occurring and thus CO should also be abundant. Model-data comparisons favor $T_{\text {eff }}=700_{-100}^{+50} \mathrm{~K}$, but the surface gravity is not well constrained (Macintosh et al. 2015). Because the planet is colder, contains more quenched $\mathrm{CH}_{4}$, and receives a stronger UV flux at its $\sim 14$-au orbital distance (De Rosa et al. 2015) than HR $8799 \mathrm{~b}$, photochemistry is expected to be more important on 51 Eri $b$, and indeed the recent independent photochemical modeling of Zahnle et al. (2016) demonstrates that this is the case.

Figure 15 shows the results for a 51 Eri b model with $T_{\text {eff }}=700 \mathrm{~K}, \log (g)=3.5 \mathrm{cgs}$ (with assumed mass $=2 M_{J}$, radius $\left.\approx 1.25 R_{J}\right), K_{\text {deep }}=2 \times 10^{6} \mathrm{~cm}^{2} \mathrm{~s}^{-1}$, and a solar metallicity, with a thermal structure derived from the radiative-convective equilibrium model described in Marley et al. (2012). We added an arbitrary $1000 \mathrm{~K}$ thermosphere to the top of this model, in an analogy with Jupiter, but we found that the presence or absence of such a thermosphere has little effect on the results. Note that this particular $K_{\text {deep }}$ value was selected because it produces a quenched $\mathrm{CH}_{4}$ abundance consistent with the absorption depths seen the Macintosh et al. (2015) spectra. Because the stratospheric temperature drops below $250 \mathrm{~K}$, water recycling is relatively inefficient (see discussion in Section 3.1 and in Zahnle et al. 2016), and as the $\mathrm{H}_{2} \mathrm{O}$ becomes depleted due to photolysis, the production of $\mathrm{CO}_{2}$ through $\mathrm{CO}+\mathrm{OH} \rightarrow \mathrm{CO}_{2}+\mathrm{H}$ proceeds prolifically. Carbon dioxide then becomes a major constituent on 51 Eri b at column abundances much greater than on HR $8799 \mathrm{~b}$. The inefficiency of water recycling also leads to greater abundances of other oxidized products such as $\mathrm{O}_{2}, \mathrm{NO}, \mathrm{H}_{2} \mathrm{CO}, \mathrm{CH}_{3} \mathrm{OH}$, and HNCO. The high UV flux, large quenched $\mathrm{CH}_{4}$ abundance, and cold stratosphere also allow greater production of complex hydrocarbons than in the HR $8799 \mathrm{~b}$ models, but again, none of the species in our models become abundant enough to condense to form hazes. The predicted $\mathrm{NH}_{3}$ abundance is significantly smaller than expected from chemical equilibrium due to the $\mathrm{N}_{2}-\mathrm{NH}_{3}$ quenching, and since $\mathrm{N}_{2}$ is more stable chemically, the photochemical production of nitrogen species is limited by this relatively low $\mathrm{NH}_{3}$ abundance. $\mathrm{HCN}$ is the dominant product of the coupled carbon-nitrogen photochemistry, but with the low derived $K_{\text {deep }}$ for this model, quenching is less important in controlling the final HCN abundance than photochemistry. The column abundances of several species from this model are provided in Table 2.

Although the disequilibrium composition of warmer young Jupiters like HR 8799 b resembles that of close-in hot Jupiters, cooler young Jupiters like 51 Eri b are in a unique regime of their own. Both photochemistry and quenching sculpt the composition, and the cooler stratospheric temperatures allow a variety of photochemical products to thrive. Carbon dioxide becomes one of the dominant atmospheric constituents, in a process that is unique to cooler young Jupiters and brown dwarfs. For stratospheres warmer than $\sim 250 \mathrm{~K}$, the $\mathrm{OH}$ released from $\mathrm{H}_{2} \mathrm{O}$ photolysis can still efficiently react with $\mathrm{H}_{2}$ to recycle the water, but this reaction slows to a trickle at low temperatures. A large percentage of the upper-stratospheric oxygen then is removed from $\mathrm{CO}$ and $\mathrm{H}_{2} \mathrm{O}$ and ends up in $\mathrm{CO}_{2}$. This process does not occur on hot Jupiters because the temperatures are too high and the water and $\mathrm{CO}$ are efficiently recycled, and it does not occur on solar-system giant planets because overall stratospheric oxygen abundances are too low as a result of water condensation in the troposphere and small external oxygen influx rates due to interplanetary dust, cometary impacts, and satellite and ring debris (e.g., Moses et al. 2004).

Based on the column abundances predicted in this model (Table 2), $\mathrm{CO}_{2}$ should be readily observable on 51 Eri $b$ in the $4.2-4.3 \mu \mathrm{m}$ and $\sim 15 \mu \mathrm{m}$ regions, and perhaps even near 2.7-2.8 $\mu \mathrm{m}$. Carbon monoxide should also be observable at 2.3-2.4 $\mu \mathrm{m}$ (given sufficient spectral resolution) and at 4.5-4.9 $\mu \mathrm{m}$. Ammonia absorption is potentially detectable in bands near $\sim 1.5,2,3$, and $6.15 \mu \mathrm{m}$ if the lines can be disentangled from other absorbers and if the photosphere extends deep enough (i.e., is not obscured by high clouds), and $\mathrm{NH}_{3}$ should be more readily detectable in the $9-11 \mu \mathrm{m}$ region. Figure 16 demonstrates that photochemical models with relatively large quenched methane abundances can reproduce the GPI spectra.

Our photochemical model results for 51 Eri $b$ are qualitatively similar to those of Zahnle et al. (2016), who use a different model, with a different numerical integrator, a different list of chemical species, different reaction rates and UV cross sections, different eddy diffusion coefficient profiles, and different assumptions about the stellar flux. Comparisons between the two models therefore give some sense of the robustness of the theoretical predictions regarding the photochemical products. The key quench reactions have different rate coefficients in the two models, so each model predicts slightly different quenched mixing ratios for any given $K_{\text {deep }}$, 
Eddy Diffusion Coefficient $\left(\mathrm{cm}^{2} \mathrm{~s}^{-1}\right)$

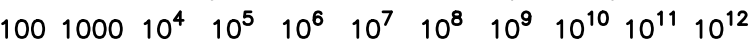
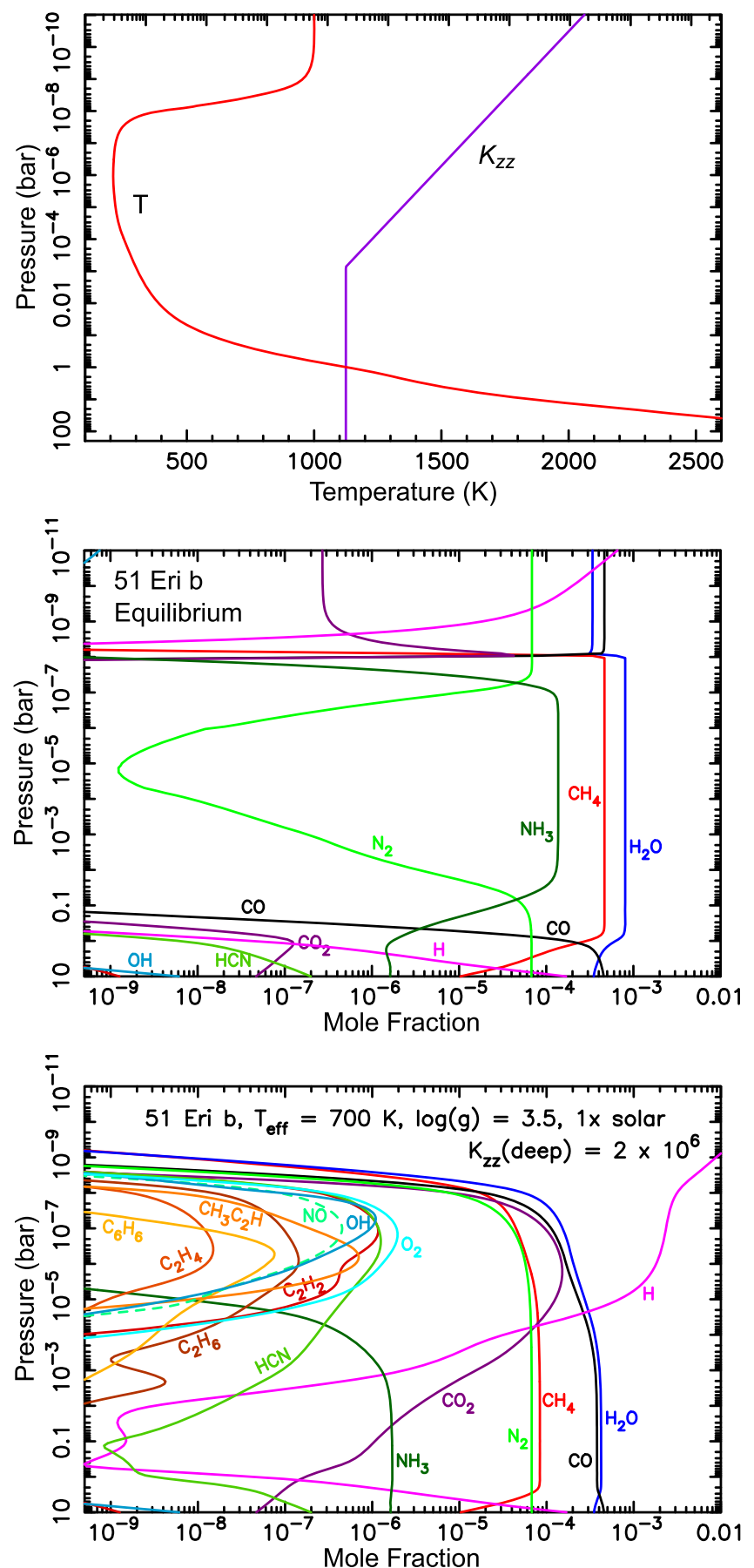

Figure 15. Chemical model for 51 Eri b assuming $T_{\text {eff }}=700 \mathrm{~K}, \log (g)=3.5$ cgs, mass $=2 M_{J}$, and solar metallicity. (Top) The temperature profile (red curve, bottom axis) from the radiative-convective equilibrium model of Marley et al. (2012) assuming the above bulk constraints, and the eddy diffusion coefficient profile (purple curve, top axis) adopted in the photochemical model; (Middle) the predicted thermochemical equilibrium mixing-ratio profiles for the major oxygen, carbon, and nitrogen species, as labeled, for the assumed pressure-temperature profile; (Bottom) mixing-ratio profiles predicted from our thermo/photochemical kinetics and transport model for the above thermal structure, $K_{z z}$ profile, and assumed bulk elemental composition.

but these differences are relatively minor (i.e., within a factor of $\sim 2$ for $\mathrm{CH}_{4}$ ). Both models predict strong photochemical production of $\mathrm{CO}_{2}$ when the stratospheric temperatures fall
Table 2

Column Abundances for 51 Eri b Models

\begin{tabular}{lccc}
\hline \hline Species & $\begin{array}{c}\text { Column } \\
\text { Abundance } \\
\text { Above } 10 \mathrm{mbar} \\
\left(\mathrm{cm}^{-2}\right)\end{array}$ & $\begin{array}{c}\text { Column } \\
\text { Abundance } \\
\text { Above } 100 \mathrm{mbar} \\
\left(\mathrm{cm}^{-2}\right)\end{array}$ & $\begin{array}{c}\text { Column } \\
\text { Abundance } \\
\text { Above } 1 \mathrm{bar} \\
\left(\mathrm{cm}^{-2}\right)\end{array}$ \\
\hline $\mathrm{CH}_{4}$ & $6.8 \times 10^{19}$ & $6.8 \times 10^{20}$ & $6.8 \times 10^{21}$ \\
$\mathrm{C}_{2} \mathrm{H}_{2}$ & $4.8 \times 10^{14}$ & $4.8 \times 10^{14}$ & $4.8 \times 10^{14}$ \\
$\mathrm{C}_{2} \mathrm{H}_{6}$ & $1.6 \times 10^{15}$ & $3.6 \times 10^{15}$ & $1.2 \times 10^{16}$ \\
$\mathrm{C}_{3} \mathrm{H}_{4}$ & $1.5 \times 10^{14}$ & $1.5 \times 10^{14}$ & $1.5 \times 10^{14}$ \\
$\mathrm{C}_{6} \mathrm{H}_{6}$ & $3.7 \times 10^{14}$ & $7.9 \times 10^{14}$ & $1.4 \times 10^{15}$ \\
$\mathrm{O}_{2}$ & $8.2 \times 10^{14}$ & $8.2 \times 10^{14}$ & $8.2 \times 10^{14}$ \\
$\mathrm{H}_{2} \mathrm{O}$ & $3.4 \times 10^{20}$ & $3.4 \times 10^{21}$ & $3.4 \times 10^{22}$ \\
$\mathrm{CO}_{\mathrm{CO}}$ & $3.0 \times 10^{20}$ & $3.1 \times 10^{21}$ & $3.1 \times 10^{22}$ \\
$\mathrm{H}_{2} \mathrm{CO}$ & $6.9 \times 10^{18}$ & $1.8 \times 10^{19}$ & $5.0 \times 10^{19}$ \\
$\mathrm{CH}_{3} \mathrm{OH}$ & $2.4 \times 10^{13}$ & $1.8 \times 10^{14}$ & $7.4 \times 10^{15}$ \\
$\mathrm{NH}_{2}$ & $6.8 \times 10^{13}$ & $8.7 \times 10^{14}$ & $2.4 \times 10^{15}$ \\
$\mathrm{NH}_{3}$ & $7.5 \times 10^{14}$ & $7.5 \times 10^{14}$ & $7.6 \times 10^{14}$ \\
$\mathrm{HCN}^{14}$ & $3.2 \times 10^{18}$ & $1.3 \times 10^{19}$ & $1.4 \times 10^{20}$ \\
$\mathrm{HC}_{3} \mathrm{~N}$ & $3.6 \times 10^{16}$ & $6.5 \times 10^{16}$ & $2.0 \times 10^{17}$ \\
$\mathrm{NO}$ & $5.1 \times 10^{13}$ & $4.9 \times 10^{14}$ & $4.9 \times 10^{14}$ \\
\end{tabular}

Note. Model assumes $\log (g)=3.5$ cgs.

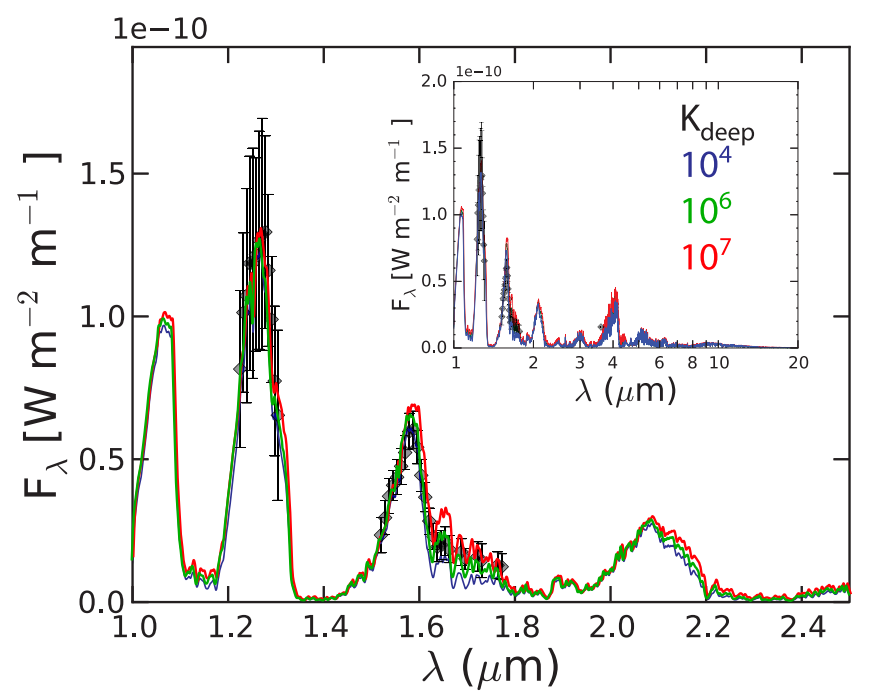

Figure 16. The 51 Eri b GPI observations of Macintosh et al. (2015) (gray/ black data points with error bars), in comparison with synthetic spectra from photochemical models that assume $T_{\text {eff }}=700 \mathrm{~K}, g=3500 \mathrm{~cm} \mathrm{~s}^{-2}$, a solar metallicity, $R \mathrm{v} 0.8 R_{J}$, and $K_{\text {deep }}=10^{4}$ (blue), $10^{4}$ (green), or $10^{7} \mathrm{~cm}^{2} \mathrm{~s}^{-1}$ (red). Lower $K_{\text {deep }}$ values lead to larger quenched $\mathrm{CH}_{4}$ abundances and greater absorption in the long-wavelength side of the $\mathrm{H}$ band. The inset shows an expanded wavelength range. As indicated by Macintosh et al. (2015), we also find that we need to invoke partial cloud cover in order to reproduce the nearinfrared observations. For this particular analysis, we combined the spectrum of a cloud-free planet with one covered by a uniform global cloud, such that the "cloud fraction" was 30\%. The cloudy model assumed a uniform gray absorbing cloud with a base at $\sim 10$ bar (representing $\mathrm{Mg}$-silicates) and an optical depth of 1.76 between 0.1 and 1000 mbar (e.g., from $\mathrm{Na}_{2} \mathrm{~S}$ clouds or photochemical haze). The planet was assumed to be $29.4 \mathrm{pc}$ from Earth.

below $\sim 250 \mathrm{~K}$, and both models predict that a variety of complex hydocarbons will be produced photochemically in the middle and upper stratosphere of 51 Eri b. The Zahnle et al. (2016) model includes sulfur chemistry, while the model presented here does not, and the list of complex hydrocarbon and nitrogen species and reactions is more extensive in the model here than in Zahnle et al. (2016). Because the nominal 
Zahnle et al. (2016) model does not contain as effective loss processes for some hydrocarbons such as $\mathrm{C}_{4} \mathrm{H}_{2}$, their model predicts greater abundances of these hydrocarbons, and Zahnle et al. (2016) presume that these hydrocarbons go on to inevitably form PAHs and organic hazes, as an upper limit to possible photochemical haze formation. The hydrocarbon chemistry presented here, which includes more $\mathrm{C}_{4} \mathrm{H}_{x}, \mathrm{C}_{5} \mathrm{H}_{x}$, and $\mathrm{C}_{6} \mathrm{H}_{x}$ hydrocarbons (including benzene) but not PAHs or heavier hydrocarbons, predicts more efficient conversion of the complex hydrocarbons back into simple hydrocarbons, $\mathrm{CO}$, $\mathrm{CO}_{2}$, and $\mathrm{HCN}$, so the effectiveness of organic haze formation on 51 Eri b has not been demonstrated. Neither model includes ion chemistry or neutral-chemistry pathways to larger organic molecules. The model here predicts a larger $\mathrm{CO}_{2}$ abundance than is obtained in Zahnle et al. (2016), for otherwise identical conditions. This result seems to stem from the presence of sulfur species in the Zahnle et al. model, where $\mathrm{H}_{2} \mathrm{~S}$ is readily destroyed by long-wavelength UV radiation, with the sulfur ending up in $\mathrm{S}_{8}$ and oxidized species, and the excess $\mathrm{H}$ released in the process helping to convert some of the $\mathrm{CO}_{2}$ back to $\mathrm{CO}$.

\section{DISCUSSION}

\subsection{Implications of Disequilibrium $\mathrm{CO}_{2}$}

Our photochemical models for generic directly imaged planets and the specific young Jupiters HR 8799 b and 51 Eri b indicate that $\mathrm{CO}_{2}$ is a major disequilibrium product on young Jupiters that is affected by both quenching and photochemistry. The $\mathrm{CO}_{2}$ abundance can increase significantly when stratospheric temperatures drop below $\sim 250 \mathrm{~K}$, when metallicities are larger than solar, and when the eddy diffusion coefficients in the troposphere and lower stratosphere are relatively small (e.g., $K_{z z}<10^{7} \mathrm{~cm}^{2} \mathrm{~s}^{-1}$ ). The $\mathrm{CO}_{2}$ produced by disequilibrium processes is likely to affect the planet's emission spectrum, especially in the $\sim 4.3$ - and $15 \mu \mathrm{m}$ regions. Detection could help constrain the planet's atmospheric metallicity, especially if $K_{z z}$ at the quench point has already been constrained from the observed relative abundance of $\mathrm{CH}_{4}$ and $\mathrm{CO}$.

Quenching (and potentially photochemistry, depending on local UV sources) will affect the $\mathrm{CO}_{2}$ abundance on brown dwarfs, as well. Brown dwarfs with lower $T_{\text {eff }}$ and colder stratospheres are expected to have more $\mathrm{CO}_{2}$ simply as a result of quenching, and the $\mathrm{CO}_{2}$ abundance can further be enhanced by photochemistry if there is a UV background sufficient to cause $\mathrm{H}_{2} \mathrm{O}$ photolysis. Perhaps galactic cosmic rays could also contribute to $\mathrm{CO}_{2}$ production if that resulting chemistry leads to a similar destruction pathway for $\mathrm{H}_{2} \mathrm{O}$, and if $\mathrm{OH}+\mathrm{H}_{2} \rightarrow$ $\mathrm{H}_{2} \mathrm{O}+\mathrm{H}$ is relatively inefficient (i.e., for cooler stratospheres). If photochemistry or cosmic-ray chemistry can lead to $\mathrm{CO}_{2}$ production on brown dwarfs, then that could explain the trends seen in the AKARI data of Yamamura et al. (2010), who find that the $\mathrm{CO}_{2}$ absorption band at $\sim 4.2-4.3 \mu \mathrm{m}$ is enhanced tremendously in cooler late $\mathrm{L}$ and $\mathrm{T}$ dwarfs.

\subsection{Implications of Disequilibrium $\mathrm{HCN}$}

Hydrogen cyanide is the second most important product of disequilibrium chemistry on young Jupiters. The HCN abundance is increased when the tropospheric $K_{\text {deep }}$ is large and the lower stratospheric $K_{z z}$ is small (i.e., a stagnant lower stratosphere overlying a convective troposphere). The strong $\mathrm{HCN}$ band near $3 \mu \mathrm{m}$ may be detectable on young Jupiters if high clouds do not fully obscure the upper troposphere, although a relatively high spectral resolution may be needed to disentangle the $\mathrm{HCN}$ lines from other absorbers such as $\mathrm{CH}_{4}$. A source of atomic $\mathrm{H}$ from $\mathrm{H}_{2} \mathrm{~S}$ and $\mathrm{PH}_{3}$ at depth (not included in this model) could lead to increased $\mathrm{HCN}$ abundances by attacking $\mathrm{CH}_{4}$ and $\mathrm{NH}_{3}$ to produce $\mathrm{CH}_{3}$ and $\mathrm{NH}_{2}$, augmenting coupled carbon-nitrogen photochemistry through $\mathrm{CH}_{3} \mathrm{NH}_{2}$ pathways such as scheme (6) above and others described more fully in Moses et al. (2010, 2011).

\subsection{Implications for Hazes}

Our neutral carbon, oxygen, and nitrogen photochemistry described here does not lead to the production of organic hazes in our young-Jupiter models. Some complex organics are produced in the models, but the abundances are not large enough in these generally warm stratospheres to lead to supersaturations. Note that the complex organics in our directly imaged planet models are less abundant than on Jupiter and Saturn, and yet the stratospheric hazes on our solar-system giant planets are not optically thick when the refractory organics such as $\mathrm{C}_{4} \mathrm{H}_{2}, \mathrm{C}_{4} \mathrm{H}_{10}$ and $\mathrm{C}_{6} \mathrm{H}_{6}$ become supersaturated and condense (e.g., Moses et al. 2004; West et al. 2004). Therefore, optically thick hydrocarbon hazes on young Jupiters might not be expected. However, ion chemistry in the auroral regions of Jupiter and Saturn seems to be more effective at producing PAHs and other complex hydrocarbons that then condense in the high-latitude stratosphere to form thicker "polar hoods" of aerosols (e.g., Pryor \& Hord 1991; Wong et al. 2000, 2003; Friedson et al. 2002).

Ion chemistry on young Jupiters may therefore enhance the production of complex hydrocarbons and eventual hazes, but even in the presence of ionization, optically thick haze formation is not guaranteed. For example, solar ionization of hydrocarbons is effective at low-to-mid latitudes on Jupiter and Saturn (e.g., Kim \& Fox 1994; Kim et al. 2014), but optically thick stratospheric photochemical hazes do not result from this process. Several Titan laboratory simulations demonstrate that $\mathrm{PAH}$ formation is favored when molecular nitrogen is present and is ionized (e.g., Imanaka \& Smith 2007). Whether this rich Titan-like ion chemistry can still occur in warmer $\mathrm{H}_{2}$ dominated situations, where the $\mathrm{CH}_{4}$ homopause limits altitude range over which the ion chemistry is effective and for which $\mathrm{O}$ and $\mathrm{OH}$ are present to potentially short-circuit the process by oxidizing the carbon and sending it preferentially to $\mathrm{CO}$ and $\mathrm{CO}_{2}$, remains to be seen. Laboratory investigations similar to those of Imanaka \& Smith 2009; Sciamma-O'Brien et al. 2010; Peng et al. 2013, and Hörst \& Tolbert (2014) but that are specifically designed for stratospheric conditions on young Jupiters would further our understanding of the likelihood of organic photochemical hazes.

Other possibilities for clouds and hazes on young Jupiters include the standard equilibrium cloud sequence (e.g., Morley et al. 2012; Marley et al. 2013), for which magnesium-silicate clouds might affect spectra if they are vertically thick, and for which $\mathrm{Na}_{2} \mathrm{~S}$ clouds are likely to reside within the photospheres of many young Jupiters (see Figure 1). Zahnle et al. (2016) identify elemental sulfur as another intriguing possible photochemical haze that is particularly likely when the stratosphere is relatively cold and well irradiated. Hydrogen sulfide is chemically fragile, and although the kinetics of sulfur species is not well determined for relevant atmospheric conditions, the formation of $S_{8}$ molecules as described by Zahnle et al. (2016) seems a likely possibility. Zahnle et al. 
(2016) find that sulfur chemistry would destroy all photospheric $\mathrm{H}_{2} \mathrm{~S}$ for a 51 Eri b planet at an orbital distance of $\lesssim$ $600 \mathrm{au}$. Phosphine $\left(\mathrm{PH}_{3}\right)$ is also a chemically fragile molecule, and the phosphorus may end up in elemental phosphorus or other relatively refractory photochemical species that could eventually form hazes. The identity of the clouds that seem to affect the spectra of young Jupiters is therefore unclear, but there are many candidate materials, including photochemical hazes.

\subsection{Implications for JWST and WFIRST}

The JWST will have the capability of obtaining high-contrast $\left(\geqslant 10^{-7}\right.$ ) images of young giant planets (Beichman et al. 2010; Clampin 2011), with well-separated ones $\left(3^{\prime \prime}-10^{\prime \prime}\right)$ being amenable to spectroscopic characterization. The Near Infrared Camera (NIRCam), Mid-Infrared Instrument (MIRI), and NearInfraRed Imager and Slitless Spectrograph (NIRISS) all have imaging modes, with MIRI and NIRCam having coronagraph options (e.g., Krist et al. 2007; Boccaletti et al. 2015; Debes et al. 2015) and NIRISS having an Aperture Masking Interferometry (AMI) mode (Artigau et al. 2014). Additionally, the JWST Near Infrared Spectrograph (NIRSpec) and MIRI instruments both have Integral Field Units (IFUs) (Arribas et al. 2007; Wells et al. 2015) that will allow for medium to high resolution $(R \sim 1000-3000)$ spectra of planets with angular separations greater than $3^{\prime \prime}$. Collectively these instruments can provide high-sensitivity photometry and spectroscopy of giant planets orbiting at a variety of separations $\left(0{ }^{\prime \prime} 1-10^{\prime \prime}\right)$ from their host stars, over the wavelength range $\sim 0.6-28 \mu \mathrm{m}$ (see the review of Beichman et al. 2010). JWST will therefore provide the opportunity for detecting the excess $\mathrm{CO}_{2}$ absorption we predict here near $\sim 4.3 \mu \mathrm{m}$ (see Figure 10) and $\sim 15 \mu \mathrm{m}$, as well as provide better constraints on the quenched methane abundance from absorption in the 3.3- and $7.7 \mu \mathrm{m}$ bands, quenched $\mathrm{NH}_{3}$ from the $10-11 \mu \mathrm{m}$ band, signatures of photochemically produced species such as $\mathrm{HCN}$ (near $\sim 3,7$, and $14 \mu \mathrm{m}$ ), $\mathrm{C}_{2} \mathrm{H}_{2}$ (at $13.6 \mu \mathrm{m}$ ), and $\mathrm{C}_{2} \mathrm{H}_{6}$ (near $12.2 \mu \mathrm{m})$.

In the next decade, NASA's Wide Field Infrared Survey Telescope (WFIRST), equipped with an optical $(0.4-0.95 \mu \mathrm{m})$ Coronagraphic Instrument (CGI), will obtain photometry and spectra for extrasolar planets with contrasts as low as $10^{-10}$ and angular separations between 0" 1 and 0."5 (Traub et al. 2016). The WFIRST CGI prime survey will target a number of planets detected via radial velocity both photometrically $(0.4-0.95 \mu \mathrm{m})$ and spectroscopically $(0.6-0.95 \mu \mathrm{m})$. These planets will be relatively cool, mature giant planets orbiting closer to their stars than Jupiter. Many of these planets will be warm enough to lack ammonia, hydrogen sulfide, and in some cases, water clouds (Marley et al. 2014). Thus their predicted photospheric equilibrium chemistry is comparable to the directly imaged planets studied here, although their deep atmospheres will be colder. Methane is likely the dominant form of carbon in the atmospheres of these planets, and although detailed predictions for the photochemistry of such worlds awaits future studies, the implications of our models presented here point to a likely rich photochemistry. Preliminary investigations (Sharp et al. 2004) suggest that the older Jupiters with neither water nor ammonia trapped in tropospheric clouds (i.e., the "Class III" planets in the terminology of, Sudarsky et al. 2003) will have stratospheric chemistry similar to what is described for our young-Jupiter models. Sharp et al. (2004) find that hydrocarbon and nitrile photochemistry is even more prevalent on "Class II" planets, for which water condenses in the troposphere but ammonia does not (Sudarsky et al. 2003), leading to high production rates for $\mathrm{HCN}, \mathrm{CH}_{3} \mathrm{CN}$, other nitriles, and complex hydrocarbons. Coupled $\mathrm{C}_{2} \mathrm{H}_{2}-\mathrm{NH}_{3}$ photochemistry will likely produce high-molecular-weight organo-nitrogen compounds (Moses et al. 2010) and PAHs. Photochemical production of hazes will likely be important, which can sculpt the ultraviolet and blue reflection spectra (e.g., Griffith et al. 1998), as on our own solar-system giant planets, thereby affecting the reflection photometry or signatures observed by WFIRST.

\section{CONCLUSIONS}

Our modeling of directly imaged exoplanets indicates that the atmospheric composition of these young Jupiters is expected to be far from chemical equilibrium, confirming the results of previous studies that indicate $\mathrm{CH}_{4}$ and $\mathrm{CO}$ quenching is occurring on these planets (e.g., Bowler et al. 2010; Hinz et al. 2010; Janson et al. 2010, 2013; Barman et al. 2011a, 2011b, 2015; Galicher et al. 2011; Marley et al. 2012; Skemer et al. 2012, 2014; Currie et al. 2014; Ingraham et al. 2014; Zahnle \& Marley 2014). Transportinduced quenching will cause $\mathrm{CO}$, and not $\mathrm{CH}_{4}$, to be the dominant carbon constituent on most lower-gravity young Jupiters with $T_{\text {eff }} \geqslant 600 \mathrm{~K}$, for all reasonable estimates of the strength of deep-atmospheric convection. This conclusion is inevitable. The first line of attack for interpreting young-Jupiter spectra should therefore always be models that include quenching. Photochemistry can also play a significant role in young-Jupiter atmospheres, especially on cooler planets that receive strong ultraviolet irradiation from their host stars.

Rapid transport in the deep atmosphere also leads to quenching of $\mathrm{H}_{2} \mathrm{O}$ at the same point as the quenching of $\mathrm{CO}$ and $\mathrm{CH}_{4}$. This effect does not appear to be as widely realized as the $\mathrm{CH}_{4}-\mathrm{CO}$ quenching phenomenon, but it is important, as the quenching can occur in a region where the equilibrium $\mathrm{H}_{2} \mathrm{O}$ mixing ratio is increasing with altitude, with quenching then causing a lower-than-expected $\mathrm{H}_{2} \mathrm{O}$ abundance on young Jupiters. In this situation, the oxygen is preferentially tied up in quenched $\mathrm{CO}$ rather than $\mathrm{H}_{2} \mathrm{O}$, and the water mixing ratio can be a factor of a few lower than equilibrium predictions. Water is the dominant infrared opacity source on young Jupiters, and the fact that quenching can alter the expected abundance can in turn affect the predicted thermal structure, cooling history, spectral energy distribution, and inferred $\mathrm{C} / \mathrm{O}$ ratio of these planets (the latter due to the fact that the $\mathrm{CO}$ abundance is typically difficult to constrain precisely). Models that consider the thermal evolution of giant planets or that predict the current thermal structure of young Jupiters should take the quenching of $\mathrm{H}_{2} \mathrm{O}$ into account, although this factor is not likely to have as large an impact as clouds or initial conditions.

Quenching will also affect the relative abundances of $\mathrm{NH}_{3}$ and $\mathrm{N}_{2}$, favoring $\mathrm{N}_{2}$ rather than $\mathrm{NH}_{3}$ at the quench point. Although $\mathrm{NH}_{3}$ is not expected to be the dominant nitrogenbearing constituent, the quenched ammonia abundance may still be observable on young Jupiters if the photosphere extends into the upper troposphere and is not obscured by clouds. The quenched $\mathrm{NH}_{3}$ mixing ratio increases as $T_{\text {eff }}$ decreases.

Other potentially observable constituents that are expected to be negligible in equilibrium models but that are affected by 
disequilibrium chemical processes include $\mathrm{CO}_{2}$ and $\mathrm{HCN}$. These molecules are affected by both quenching and photochemistry. The quenching process leads to increases in the HCN abundance when deep atmospheric mixing is strong, while increases in $\mathrm{CO}_{2}$ are favored when deep atmospheric mixing is weak. Photochemical production of both HCN and $\mathrm{CO}_{2}$ is more important for weak lower-stratospheric mixing and strong UV irradiation. Effective temperatures of 900-1400 $\mathrm{K}$ favor larger $\mathrm{HCN}$ column abundances, whereas the $\mathrm{CO}_{2}$ column abundance increases significantly for lower $T_{\text {eff }}$, and specifically for low stratospheric temperatures $T \lesssim 250 \mathrm{~K}$. When stratospheric temperatures are low, the reaction $\mathrm{OH}+\mathrm{H}_{2}$ $\rightarrow \mathrm{H}_{2} \mathrm{O}+\mathrm{H}$ becomes ineffective, and $\mathrm{OH}+\mathrm{CO} \rightarrow \mathrm{CO}_{2}+\mathrm{H}$ can compete (see also Zahnle et al. 2016), depleting the upper stratospheric $\mathrm{H}_{2} \mathrm{O}$ and $\mathrm{CO}$, and significantly increasing the column abundance of photochemically produced $\mathrm{CO}_{2}$. On cooler planets like 51 Eri b, the $\mathrm{CO}_{2}$ peak mixing ratio can even exceed that of $\mathrm{CH}_{4}$ and rivals that of $\mathrm{CO}$ and $\mathrm{H}_{2} \mathrm{O}$ in the upper stratosphere. Carbon dioxide is likely to be observable on all young Jupiters with moderate-to-low atmospheric mixing, but will be especially important on cooler planets. Hydrogen cyanide is less likely to be observable on young Jupiters, but it may be detectable in the $\sim 3 \mu \mathrm{m}$ band given favorable atmospheric conditions (including the absence of high clouds) and sufficient spectral resolution to disentangle the lines from other nearby absorbers.

Complex hydrocarbons also form photochemically on young Jupiters, but the generally warm stratospheric temperatures and large $\mathrm{H}$ abundance make them less stable than on the giant planets in our solar system. Oxidation of the carbon to form $\mathrm{CO}$ and $\mathrm{CO}_{2}$ also competes effectively, unlike on our own giant planets. It is unlikely that hydrocarbons produced from neutral photochemistry will be observable on young Jupiters. Note that the models presented here include only $\mathrm{H}-, \mathrm{C}-, \mathrm{O}-$, and $\mathrm{N}$-bearing species. Ion chemistry is not included, nor is the neutral photochemistry of other volatiles like sulfur and phosphorus. As shown by Zahnle et al. (2016), sulfur chemistry can alter some of the predictions regarding the abundances of $\mathrm{C}-, \mathrm{N}-$, and $\mathrm{O}-$ species. Although organic hazes do not form from the neutral chemistry considered here, ion chemistry may augment the production of refractory organics, as on Titan and in the auroral regions of Jupiter (e.g., Wong et al. 2000; Vuitton et al. 2007; Waite et al. 2007). Future laboratory and theoretical modeling should focus on this possibility. Laboratory studies that investigate the kinetics of $\mathrm{C}_{3} \mathrm{H}_{2}$ and $\mathrm{C}_{3} \mathrm{H}_{3}$ reactions with other hydrocarbon radicals and molecules would aid exoplanet photochemistry studies. Other possible photochemically produced hazes include elemental sulfur (Zahnle et al. 2016), elemental phosphorus or other refractory phosphorus species, and refractory products from coupled $\mathrm{C}_{2} \mathrm{H}_{2}-\mathrm{NH}_{3}$ chemistry (e.g., Keane et al. 1996; Ferris \& Ishikawa 1988; Moses et al. 2010).

Detection and abundance determinations for key molecules like $\mathrm{CH}_{4}, \mathrm{H}_{2} \mathrm{O}, \mathrm{CO}, \mathrm{CO}_{2}$, and $\mathrm{NH}_{3}$ can help constrain planetary properties and potentially break other modeling degeneracies. The $\mathrm{CH}_{4}$ and $\mathrm{NH}_{3}$ mixing ratios are strong indicators of the strength of deep atmospheric mixing, $K_{\text {deep }}$, as well as the planet's effective temperature, $T_{\text {eff }}$. Simultaneous measurements of the abundance of $\mathrm{H}_{2} \mathrm{O}$ and $\mathrm{CO}$ can provide additional constraints on $T_{\text {eff }}$, surface gravity, and metallicity. The $\mathrm{CO}_{2}$ abundance is very sensitive to metallicity (e.g., Lodders \& Fegley 2002; Moses et al. 2013a), and can also become quite large for low $T_{\text {eff }}$, low stratospheric $K_{z z}$, and high UV irradiance.

The disequilibrium composition of warmer young Jupiters (i.e., $T_{\text {eff }} \gtrsim 900 \mathrm{~K}$ ), such as HR $8799 \mathrm{~b}$, resembles that of close-in transiting giant planets. Transport-induced quenching is the dominant process driving the atmospheres out of equilibrium, and the stratospheres are too warm to allow many of the photochemical products to survive, other than molecules with strong bonds like $\mathrm{C}_{2} \mathrm{H}_{2}$ and $\mathrm{HCN}$. However, cooler young Jupiters $\left(T_{\text {eff }} \lesssim 700 \mathrm{~K}\right)$ like 51 Eri b can have a rich and interesting photochemistry that differs distinctly from that of either hot Jupiters or the cold giant planets in our solar system. The quenched abundances of photochemically active $\mathrm{CH}_{4}$ and $\mathrm{NH}_{3}$ tend to be greater for lower $T_{\text {eff }}$, and hydrocarbon photochemical products survive more readily when stratospheric temperatures are low. Oxidation of the carbon and nitrogen species can also proceed much more effectively when stratospheric temperatures are low (due to a reduction in efficiency of $\mathrm{H}_{2} \mathrm{O}$ recycling), leading to oxidized products like $\mathrm{NO}, \mathrm{O}_{2}$, and especially $\mathrm{CO}_{2}$. Carbon dioxide is likely to be a major absorber on cooler young Jupiters.

Cooler directly imaged giant planets that receive moderateto-high UV flux from their host stars fall into a unique and interesting chemical regime that is controlled by both transportinduced quenching and an active, rich photochemistry. This chemical regime has no representation in our own solar system because the terrestrial planets have very different atmospheric compositions and the colder giant planets have key oxygen and nitrogen species tied up in condensates at depth, so that coupled nitrogen-carbon, oxygen-carbon, and nitrogen-oxygen photochemistry is suppressed. The simultaneous presence of $\mathrm{H}_{2} \mathrm{O}, \mathrm{CO}, \mathrm{CH}_{4}, \mathrm{~N}_{2}$, and $\mathrm{NH}_{3}$ on cooler young Jupiters leads to complex photochemical interactions with both oxidized and reduced products being stable, and small amounts of highmolecular-weight pre-biotic molecules being able to form and survive. With dedicated ground-based campaigns ramping up their search for young Jupiters (e.g., Macintosh et al. 2015; Tamura 2016; Vigan et al. 2016), and missions such as JWST, GAIA, and WFIRST gearing up or being planned, we look forward to many future reports of the atmospheric composition of directly imaged giant planets.

This material is based upon work supported by the National Aeronautics and Space Administration through NASA Exoplanet Research Program grant NNX15AN82G (initially) and NNX16AC64G. We thank Kevin France for useful advice on constructing the stellar ultraviolet fluxes, and the anonymous reviewer for a thorough review of the manuscript. Portions of the stellar spectra were compiled using data from the Mikulski Archive for Space Telescopes (MAST) at STSci and the $\mathrm{X}$-exoplanet archive at the CAB.

\section{REFERENCES}

Agúndez, M., Parmentier, V., Venot, O., Hersant, F., \& Selsis, F. 2014a, A\&A, 564, A73

Agúndez, M., Venot, O., Selsis, F., \& Iro, N. 2014b, ApJ, 781, 68

Allard, F., Hauschildt, P. H., Alexander, D. R., Tamanai, A., \& Schweitzer, A. 2001, ApJ, 556, 357

Allen, M., Yung, Y. L., \& Waters, J. W. 1981, JGR, 86, 3617

Arribas, S., Ferruit, P., Jakobsen, P., et al. 2007, in Science Perspectives for 3D Spectroscopy, ed. M. Kissler-Patig, J. R. Walsh, \& M. M. Roth (Berlin: Springer), 21

Artigau, É., Sivaramakrishnan, A., Greenbaum, A. Z., et al. 2014, Proc. SPIE, 9143,914340 
Atkinson, D. B., \& Hudgens, J. 1999, JPCA, 103, 4242

Atreya, S. K., Donahue, T. M., Nagy, A. F., Waite, J. H., Jr., \& McConnell, J. C. 1984, in Saturn, ed. T. Gehrels \& M. S. Matthews (Tucson, AZ: Univ. Arizona Press), 239

Atreya, S. K., \& Romani, P. N. 1985, in Recent Advances in Planetary Meteorology, ed. G. E. Hunt (Cambridge: Cambridge Univ. Press)

Bailey, J. 2014, PASA, 31, e043

Barman, T. S., Konopacky, Q. M., Macintosh, B., \& Marois, C. 2015, ApJ, 804, 61

Barman, T. S., Macintosh, B., Konopacky, Q. M., \& Marois, C. 2011a, ApJ, 733, 65

Barman, T. S., Macintosh, B., Konopacky, Q. M., \& Marois, C. 2011b, ApJL, 735, L39

Baudino, J.-L., Bézard, B., Boccaletti, A., et al. 2015, A\&A, 582, A83

Baulch, D. L., Bowman, C. T., Cobos, C. J., et al. 2005, JPCRD, 34, 757

Beichman, C. A., Krist, J., Trauger, J. T., et al. 2010, PASP, 122, 162

Benneke, B. 2015, arXiv:1504.07655

Bézard, B., Lellouch, E., Strobel, D., Maillard, J.-P., \& Drossart, P. 2002, Icar, 159,95

Boccaletti, A., Lagage, P.-O., Baudoz, P., et al. 2015, PASP, 127, 633

Bonnefoy, M., Boccaletti, A., Lagrange, A.-M., et al. 2013, A\&A, 555, A107

Bonnefoy, M., Zurlo, A., Baudino, J. L., et al. 2016, A\&A, 587, A58

Bowler, B. P. 2016, PASP, 128, 102001

Bowler, B. P., Liu, M. C., Dupuy, T. J., \& Cushing, M. C. 2010, ApJ, 723, 850

Brasseur, G. P., Orlando, J. J., \& Tyndall, G. S. 1999, Atmospheric Chemistry and Global Change (New York: Oxford Univ. Press)

Burrows, A., Sudarsky, D., \& Lunine, J. I. 2003, ApJ, 596, 587

Castelli, F., \& Kurucz, R. L. 2004, arXiv:astro-ph/0405087

Chilcote, J., Barman, T., Fitzgerald, M. P., et al. 2015, ApJL, 798, L3

Clampin, M. 2011, in Proc. IAU Symp. 276, The Astrophysics of Planetary Systems: Formation, Structure, and Dynamical Evolution, ed. A. Sozzetti,

M. G. Lattanzi, \& A. P. Boss (Cambridge: Cambridge Univ. Press), 335

Crossfield, I. J. M. 2015, PASP, 127, 941

Currie, T., Burrows, A., Girard, J. H., et al. 2014, ApJ, 795, 133

Currie, T., Burrows, A., Itoh, Y., et al. 2011, ApJ, 729, 128

De Rosa, R. J., Nielsen, E. L., Blunt, S. C., et al. 2015, ApJL, 814, L3

Dean, A. M., Chou, M.-S., \& Stern, D. 1984, Int. J. Chem. Kinet., 16, 633

Debes, J. H., Ygouf, M., Choquet, E., et al. 2015, arXiv:1511.06277

Dobrijevic, M., Cavalié, T., Hébrard, E., et al. 2010, P\&SS, 58, 1555

Fahr, A., \& Nayak, A. 2000, Int. J. Chem. Kinet., 32, 118

Fegley, B., Jr., \& Lodders, K. 1994, Icar, 110, 117

Ferris, J. P., \& Ishikawa, Y. 1988, JAChS, 110, 4306

Fortney, J. J., Marley, M. S., Saumon, D., \& Lodders, K. 2008b, ApJ, 683, 1104

Fouchet, T., Moses, J. I., \& Conrath, B. J. 2009, in Saturn from CassiniHuygens, ed. M. K. Dougherty, L. W. Esposito, \& S. M. Krimigis (Berlin: Springer), 83

Freytag, B., Allard, F., Ludwig, H.-G., Homeier, D., \& Steffen, M. 2010, A\&A, 513, A19

Friedson, A. J., Wong, A.-S., \& Yung, Y. L. 2002, Icar, 158, 389

Galicher, R., Marois, C., Macintosh, B., Barman, T., \& Konopacky, Q. 2011, ApJL, 739, L41

Gordon, S., \& McBride, B. J. 1994, NASA Reference Publ., 1311

Grevesse, N., Asplund, M., \& Sauval, A. 2007, SSRv, 130, 105

Griffith, C. A., \& Yelle, R. V. 1999, ApJL, 519, L85

Griffith, C. A., Yelle, R. V., \& Marley, M. S. 1998, Sci, 282, 2063

Hauschildt, P. H., Baron, E., \& Allard, F. 1997, ApJ, 483, 390

Heap, S. R., \& Lindler, D. 2011, in ASP Conf. Ser. 448, 16th Cambridge Workshop on Cool Stars, Stellar Systems, and the Sun, ed. C. Johns-Krull,

M. K. Browning, \& A. A. West (San Francisco, CA: ASP), 887

Hébrard, E., Dobrijevic, M., Loison, J. C., et al. 2013, A\&A, 552, A132

Heng, K., Lyons, J. R., \& Tsai, S.-M. 2016, ApJ, 96, 96

Hidaka, Y., Oki, Y., \& Kawano, H. 1989, JPhCh, 93, 7134

Hinz, P. M., Rodigas, T. J., Kenworthy, M. A., et al. 2010, ApJ, 716, 417

Hörst, S. M., \& Tolbert, M. A. 2014, ApJ, 781, 53

Hörst, S. M., Yelle, R. V., Buch, A., et al. 2012, AsBio, 12, 809

Hu, R., \& Seager, S. 2014, ApJ, 784, 63

Hubeny, I., \& Burrows, A. 2007, ApJ, 669, 1248

Imanaka, H., \& Smith, M. A. 2007, GeoRL, 34, L02204

Imanaka, H., \& Smith, M. A. 2009, JPCA, 113, 11187

Ingraham, P., Marley, M. S., Saumon, D., et al. 2014, ApJL, 794, L15

Janson, M., Bergfors, C., Goto, M., Brandner, W., \& Lafrenière, D. 2010 ApJL, 710, L35

Janson, M., Brandt, T. D., Kuzuhara, M., et al. 2013, ApJL, 778, L4

Jasper, A. W., Klippenstein, S. J., Harding, L. B., \& Ruscic, B. 2007, JPCA, 111,3932
Kaye, J. A., \& Strobel, D. F. 1983, Icar, 55, 399

Keane, T. C., Yuan, F., \& Ferris, J. P. 1996, Icar, 122, 205

Kim, Y. H., \& Fox, J. L. 1994, Icar, 112, 310

Kim, Y. H., Fox, J. L., Black, J. H., \& Moses, J. I. 2014, JGR, 119, 384

Klippenstein, S. J., Harding, L. B., Ruscic, B., et al. 2009, JPCA, 113, 10241

Knyazev, V. D., \& Slagle, I. R. 2001, JPCA, 105, 3196

Konnov, A. A., \& De Ruyck, J. 2000, CST, 152, 23

Konopacky, Q. M., Barman, T. S., Macintosh, B. A., \& Marois, C. 2013, Sci, 339, 1398

Kopparapu, R. k., Kasting, J. F., \& Zahnle, K. J. 2012, ApJ, 745, 77

Krist, J. E., Beichman, C. A., Trauger, J. T., et al. 2007, Proc. SPIE, 6693, $66930 \mathrm{H}$

Landsman, W., \& Simon, T. 1993, ApJ, 408, 305

Lavvas, P., Koskinen, T., \& Yelle, R. V. 2014, ApJ, 796, 15

Lee, J.-M., Heng, K., \& Irwin, P. G. J. 2013, ApJ, 778, 97

Lendvay, G., Bérces, T., \& Márta, F. 1997, JPCA, 101, 1588

Lewis, J. S., \& Fegley, M. B., Jr. 1984, SSRv, 39, 163

Liang, M.-C., Parkinson, C. D., Lee, A. Y.-T., Yung, Y. L., \& Seager, S. 2003, ApJL, 596, L247

Lindzen, R. S. 1981, JGR, 86, 9707

Line, M. R., Fortney, J. J., Marley, M. S., \& Sorahana, S. 2014, ApJ, 793, 33

Line, M. R., Teske, J., Burningham, B., Fortney, J. J., \& Marley, M. S. 2015, ApJ, 807, 183

Line, M. R., Vasisht, G., Chen, P., Angerhausen, D., \& Yung, Y. L. 2011, ApJ, 738,32

Line, M. R., Wolf, A. S., Zhang, X., et al. 2013, ApJ, 775, 137

Lodders, K. 2010, in Formation and Evolution of Exoplanets, ed. R. Barnes (Berlin: Wiley), 157

Lodders, K., \& Fegley, B. 2002, Icar, 155, 393

Macintosh, B., Graham, J. R., Barman, T., et al. 2015, Sci, 350, 64

Madhusudhan, N., Agúndez, M., Moses, J. I., \& Hu, Y. 2016, SSRv, in press doi:10.1007/s11214-016-0254-3

Madhusudhan, N., Burrows, A., \& Currie, T. 2011, ApJ, 737, 34

Madhusudhan, N., Knutson, H., Fortney, J. J., \& Barman, T. 2014, in Protostars and Planets VI, ed. H. Beuther et al. (Tucson, AZ: Univ. Arizona Press), 739

Maire, A.-L., Skemer, A. J., Hinz, P. M., et al. 2015, A\&A, 576, A133

Marley, M., Lupu, R., Lewis, N., et al. 2014, arXiv:1412.8440

Marley, M. S., Ackerman, A. S., Cuzzi, J. N., \& Kitzmann, D. 2013, in Comparative Climatology of Terrestrial Planets, ed. S. J. Mackwell et al. (Tucson, AZ: Univ. Arizona Press), 367

Marley, M. S., Fortney, J., Seager, S., \& Barman, T. 2007, in Protostars and Planets V, ed. B. Reipurth, D. Hewitt, \& K. Keil (Tucson, AZ: Univ. Arizona Press), 733

Marley, M. S., Gelino, C., Stephens, D., Lunine, J. I., \& Freedman, R. 1999, ApJ, 513, 879

Marley, M. S., Saumon, D., Cushing, M., et al. 2012, ApJ, 754, 135

Marley, M. S., Seager, S., Saumon, D., et al. 2002, ApJ, 568, 335

Marois, C., Macintosh, B., Barman, T., et al. 2008, Sci, 322, 1348

Marois, C., Zuckerman, B., Konopacky, Q. M., Macintosh, B., \& Barman, T. 2010, Natur, 468, 1080

McKay, C. P., Pollack, J. B., \& Courtin, R. 1989, Icar, 80, 23

Miguel, Y., \& Kaltenegger, L. 2014, ApJ, 780, 166

Miguel, Y., Kaltenegger, L., Linsky, J. L., \& Rugheimer, S. 2015, MNRAS, 446, 345

Millar, T. J., Bennett, A., Rawlings, J. M. C., Brown, P. D., \& Charnley, S. B 1991, A\&AS, 87, 585

Miller-Ricci Kempton, E., Zahnle, K., \& Fortney, J. J. 2012, ApJ, 745, 3

Morley, C. V., Fortney, J. J., Kempton, E. M.-R., et al. 2013, ApJ, 775, 33

Morley, C. V., Fortney, J. J., Marley, M. S., et al. 2012, ApJ, 756, 172

Morzinski, K. M., Males, J. R., Skemer, A. J., et al. 2015, ApJ, 815, 108

Moses, J. I. 2014, RSPTA, 372, 20130073

Moses, J. I., Armstrong, E. S., Fletcher, L. N., et al. 2015, Icar, 261, 149

Moses, J. I., Bézard, B., Lellouch, E., et al. 2000, Icar, 143, 244

Moses, J. I., Fouchet, T., Bézard, B., et al. 2005, JGR, 110, E08001

Moses, J. I., Fouchet, T., Yelle, R. V., et al. 2004, in Jupiter. The Planet, Satellites and Magnetosphere, ed. F. Bagenal, T. E. Dowling, \& W. B. McKinnon (Cambridge: Cambridge Univ. Press), 129

Moses, J. I., Line, M. R., Visscher, C., et al. 2013a, ApJ, 777, 34

Moses, J. I., Madhusudhan, N., Visscher, C., \& Freedman, R. S. 2013b, ApJ, 763,25

Moses, J. I., Rages, K., \& Pollack, J. B. 1995, Icar, 113, 232

Moses, J. I., Visscher, C., Fortney, J. J., et al. 2011, ApJ, 737, 15

Moses, J. I., Visscher, C., Keane, T. C., \& Sperier, A. 2010, FaDi, 147, 103 
Nagy, A. F., Kliore, A. J., Mendillo, M., et al. 2009, in Saturn from CassiniHuygens, ed. M. K. Dougherty, L. W. Esposito, \& S. M. Krimigis (Dordrecht: Springer), 181

Norton, T. S., \& Dryer, F. L. 1989, CST, 63, 107

Norton, T. S., \& Dryer, F. L. 1990, Int. J. Chem. Kinet., 22, 219

Öberg, K. I., Murray-Clay, R., \& Bergin, E. A. 2011, ApJL, 743, L16

Oppenheimer, B. R., Baranec, C., Beichman, C., et al. 2013, ApJ, 768, 24

Orton, G. S., Moses, J. I., Fletcher, L. N., et al. 2014, Icar, 243, 471

Parmentier, V., Showman, A. P., \& Lian, Y. 2013, A\&A, 558, A91

Patience, J., King, R. R., de Rosa, R. J., \& Marois, C. 2010, A\&A, 517, A76

Peng, Z., Gautier, T., Carrasco, N., et al. 2013, JGR, 118, 778

Prinn, R. G., \& Barshay, S. S. 1977, Sci, 198, 1031

Pryor, W. R., \& Hord, C. W. 1991, Icar, 91, 161

Rajan, A., Barman, T., Soummer, R., et al. 2015, ApJL, 809, L33

Rimmer, P. B., \& Helling, C. 2016, ApJS, 224, 9

Rimmer, P. B., Helling, C., \& Bilger, C. 2014, IJAsB, 13, 173

Romani, P. N., Bishop, J., Bezard, B., \& Atreya, S. 1993, Icar, 106, 442

Sanz-Forcada, J., Micela, G., Ribas, I., et al. 2011, A\&A, 532, A6

Saumon, D., \& Marley, M. S. 2008, ApJ, 689, 1327

Sciamma-O'Brien, E., Carrasco, N., Szopa, C., Buch, A., \& Cernogora, G. 2010, Icar, 209, 704

Sharp, A. G., Moses, J. I., Friedson, A. J., et al. 2004, LPSC, 35, 1142

Sims, I. R., Queffelec, J.-L., Travers, D., et al. 1993, CPL, 211, 461

Skemer, A. J., Hinz, P. M., Esposito, S., et al. 2012, ApJ, 753, 14

Skemer, A. J., Marley, M. S., Hinz, P. M., et al. 2014, ApJ, 792, 17

Smith, M. D. 1998, Icar, 132, 176

Snellen, I. A. G., Brandl, B. R., de Kok, R. J., et al. 2014, Natur, 509, 63

Spiegel, D. S., \& Burrows, A. 2012, ApJ, 745, 174

Stone, P. H. 1976, in Jupiter, ed. T. Gehrels (Tucson, AZ: Univ. Arizona Press), 586

Strobel, D. F. 1981, JGR, 86, 9806

Strobel, D. F. 1983, IRPC, 3, 145

Sudarsky, D., Burrows, A., \& Hubeny, I. 2003, ApJ, 588, 1121
Summers, M. E., \& Strobel, D. F. 1989, ApJ, 346, 495

Tamura, M. 2016, PJAB, 92, 45

Traub, W. A., Breckinridge, J., Greene, T. P., et al. 2016, JATIS, 2, 011020

Venot, O., Fray, N., Bénilan, Y., et al. 2013, A\&A, 551, A131

Venot, O., Hébrard, E., Agúndez, M., et al. 2012, A\&A, 546, A43

Vigan, A., Bonnefoy, M., Ginski, C., et al. 2016, A\&A, 587, A55

Visscher, C., \& Moses, J. I. 2011, ApJ, 738, 72

Visscher, C., Moses, J. I., \& Saslow, S. A. 2010b, Icar, 209, 602

Vuitton, V., Yelle, R. V., \& McEwan, M. J. 2007, Icar, 191, 722

Waite, J. H., Young, D. T., Cravens, T. E., et al. 2007, Sci, 316, 870

Wang, D., Gierasch, P. J., Lunine, J. I., \& Mousis, O. 2015, Icar, 250, 154

Wells, M., Pel, J.-W., Glasse, A., et al. 2015, PASP, 127, 646

West, R. A., Baines, K. H., Friedson, A. J., et al. 2004, in Jupiter. The Planet, Satellites and Magnetosphere, ed. F. Bagenal, T. E. Dowling, \& W. B. McKinnon (Cambridge: Cambridge Univ. Press), 79

Wong, A.-S., Lee, A. Y. T., Yung, Y. L., \& Ajello, J. M. 2000, ApJL, 534, L215

Wong, A.-S., Yung, Y. L., \& Friedson, A. J. 2003, GeoRL, 30, 1447

Woods, T. N., \& Rottman, G. J. 2002, in Atmospheres in the Solar System: Comparative Aeronomy, Geophysical Monograph 130, ed. M. Mendillo, A. Nagy, \& J. H. Waite (Washington, DC: American Geophysical Union), 221

Yamamura, I., Tsuji, T., \& Tanabé, T. 2010, ApJ, 722, 682

Yelle, R. V., \& Miller, S. 2004, in Jupiter. The Planet, Satellites and Magnetosphere, ed. F. Bagenal, T. E. Dowling, \& W. B. McKinnon (Cambridge: Cambridge Univ. Press), 185

Yung, Y. L., Allen, M., \& Pinto, J. P. 1984, ApJS, 55, 465

Yung, Y. L., \& DeMore, W. B. 1999, Photochemistry of Planetary Atmospheres (Oxford: Oxford Univ. Press)

Zahnle, K., Marley, M. S., \& Fortney, J. J. 2009, arXiv:0911.0728

Zahnle, K., Marley, M. S., Morley, C. V., \& Moses, J. I. 2016, ApJ, 825, 137

Zahnle, K. J., \& Marley, M. S. 2014, ApJ, 797, 41

Zurlo, A., Vigan, A., Galicher, R., et al. 2016, A\&A, 587, A57 